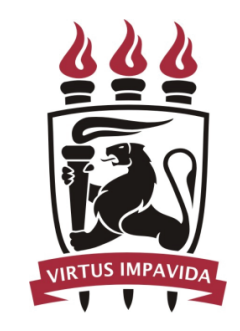

Universidade Federal de Pernambuco

Centro de Ciências Exatas e da Natureza

Centro de Informática

Pós-graduação em Ciência da Computação

\title{
A framework for monitorable services implementation
}

David Menezes Cardoso

Dissertação de Mestrado

Recife

Fevereiro, 2012. 



\author{
Universidade Federal de Pernambuco \\ Centro de Ciências Exatas e da Natureza \\ Centro de Informática
}

David Menezes Cardoso

\title{
A framework for monitorable services implementation
}

Trabalho apresentado ao Programa de Pós-graduação em Ciência da Computação do Centro de Informática da Universidade Federal de Pernambuco como requisito parcial para obtenção do grau de Mestre em Engenharia de Software.

Orientador: Augusto Cesar Alves de Sampaio

Recife

Fevereiro, 2012. 
Catalogação na fonte

Bibliotecária Jane Souto Maior, CRB4-571

Cardoso, David Menezes

A framework for monitorable services implementation / David Menezes Cardoso. - Recife: O Autor, 2012.

xxii, 126 p. : il., fig., tab.

Orientador: Augusto Cesar Alves de Sampaio.

Dissertação (mestrado) - Universidade Federal de Pernambuco. CIn, Ciência da Computação, 2012.

Inclui bibliografia.

1. Engenharia de software. 2. Framework. 3. Reuso de software. 4. Qualidade de software. I. Sampaio, Augusto Cesar Alves de (orientador). II. Título.

005.1

CDD (23. ed.)

MEI2012 - 053 
Dissertação de Mestrado apresentada por David Menezes Cardoso à Pós-Graduação em Ciência da Computação do Centro de Informática da Universidade Federal de Pernambuco, sob o título "Um Framework para Implementação de Serviços Monitoráveis" orientada pelo Prof. Augusto Cesar Alves Sampaio e aprovada pela Banca Examinadora formada pelos professores:

Prof. André Luís de Medeiros Santos

Centro de Informática / UFPE

Prof. Tiago Lima Massoni

Departamento de Sistemas e Computação / UFCG

Prof. Augusto Cesar Alves Sampaio

Centro de Informática/ UFPE

Visto e permitida a impressão.

Recife, 16 de fevereiro de 2012.

\section{Prof. Nelson Souto Rosa}

Coordenador da Pós-Graduação em Ciência da Computação do

Centro de Informática da Universidade Federal de Pernambuco. 

To my family. 



\section{Acknowledgements}

I am very thankful to everyone who aided me all along this work development. They contributed in different ways and were all important for this work to complete.

First, I thank my advisor, Professor Augusto Sampaio, for trusting in my potential, providing me with his knowledge and guidance along this work development. I also thank him for his vast patience in correcting my many technical mistakes and omissions, not to mention the many english mistakes and misuses. Finally, I thank him for being flexible and tolerant regarding my time availability, giving me the amount of time I needed to show new results.

I thank Professor Alexandre Vasconcelos for evaluating my graduation work (which served as a sketch for this work) and contributing with the idea of elaborating a chapter for discussing software development process guidelines. I also thank Professor Paulo Borba for teaching me the aspect-oriented programming concepts and helping me analyze how it can deal with the monitoring problem. I must also thank Professors André Santos and Tiago Massoni, for reviewing the dissertation and providing valuable critics and contributions which led to significant improvements to the final work.

I thank Vitor Braga for sending me valuable information regarding the Empirical Software Engineering discipline, which proved essential for conducting the case study and evaluating the work. I also thank Sidney Nogueira for introducing me to the $\mathrm{IAT}_{\mathrm{E}} \mathrm{X}$ language syntax, which I used to write the dissertation.

I thank the development team of SUATI, for their effort collecting archived data from completed projects, using code metric tools, counting lines of code, measuring execution times and helping me collect the data for the case study. It was a huge test of patience. I promise to reward their efforts. ;) For that, I thank Arthur Rizzo, Cleivson Arruda, Deaulas Neto, Fernando Spanghero, Iúri dos Anjos, Rodrigo Belo and Sérgio René.

I thank Rebeca Kramer, my girlfriend, for her comprehension and support along these years, when the weekends and holidays got compromised by the dissertation work. She was my companion while I wrote part of these chapters - certainly the best parts, since she is a source of inspiration to me.

Finally, I thank my family, for the genuine interest in my personal growth, always encouraging me to face challenges, overcome difficulties, take responsibilities, never stop learning, work hard and still have fun. A special thanks to Daniel, my older brother, which also happens to be my business partner, thanks for injecting me with confidence when I was worried about whether or not I would be able to complete the dissertation, and also for covering me when I had to stay out of the office. I also thank my younger sister, Débora, for saving me time by drawing a dozen process model diagrams. Finally, I must also thank my grandmother for her prayers - I think they worked. :) 

There may be fairies at the bottom of the garden. There is no evidence for it, but you can't prove that there aren't any, so shouldn't we be agnostic with respect to fairies? -RICHARD DAWKINS 



\section{Abstract}

Since the very first graphical user interfaces, progress indicators have been widely used to provide feedback regarding the execution of a system long-running tasks. In fact, practical experience and formal experiments suggest that such indicators are an important user interface tool, as they enhance the attractiveness and effectiveness of the programs that incorporate them. However, in order to make progress feedback possible, the system services involved must provide on-line monitoring capabilities. As the software systems become increasingly larger and more complex - often involving complex interactions between various different components and abstraction layers - , the crosscutting nature of monitoring concerns can introduce several inherent challenges to the software development: (1) code quality degradation with respect to tangling and scattering; (2) costly software evolution and maintenance difficulties; (3) absence of specific development patterns and regular standardized process guidance; (4) loss of development productivity; and (5) inconsistent monitoring results.

In this context, this work provides an analysis of monitoring requirements, possible approaches towards its implementation along with an analysis of the main benefits and weaknesses involved. Furthermore, it is proposed and evaluated a solution to aid the software development by overcoming the monitoring-related inherent challenges, rather by mitigating or completely eliminating the problems. The solution consists of a framework, extended libraries, and generic software process guidelines regarding the monitoring requirements, with focus on the Rational Unified Process (RUP), for exemplification purpose, but not limited to it.

Keywords: service execution monitoring, percent-done indicators, progress indicators, code tangling, code scattering, crosscutting concern 



\section{Resumo}

Desde as primeiras interfaces gráficas, indicadores de progresso têm sido amplamente utilizados em sistemas de informação, sendo aplicados a diferentes contextos em que o tempo necessário para completar alguma execução não seja suficientemente curto. Estudos indicam - e, de fato, a prática industrial corrobora - que esses indicadores são uma importante ferramenta de interfaces, melhorando a experiência do usuários e a atratividade dos sistemas que os utilizam. Entretanto, para que tais indicadores de progresso possam ser implementados, todos os serviços e workflows que contribuam significativamente para o tempo de execução precisam oferecer suporte a um tipo de monitorimento durante sua execução. Com os sitemas de informação se tornando cada vez maiores e sofisticados - possivelmente envolvendo um complexo emaranhado de interações entre vários componentes distintos e camadas de abstração —, a natureza crosscutting do requisito de monitoramento de serviços pode trazer algumas dificuldades para o desenvolvimento desses sistemas: (1) degradação da qualidade do código, com efeito no aumento de code tangling e scattering; (2) aumento nos custos de evolução e manutenção dos sistemas; (3) ausência de padrões de projeto específicos para guiar o processo de desenvolvimento; (4) redução de produtividade de implementação dos serviços; e (5) resultados inconsistentes gerados pelo monitoramento.

Nesse contexto, este trabalho apresenta uma análise dos requisitos de monitoramento, possíveis abordagens de implementação e uma análise dos principais pontos fortes e fracos. Em seguida, uma solução para dar suporte à implementação de serviços monitoráveis, com o objetivo de mitigar ou eliminar as principais dificuldades relacionadas, é apresentada e avaliada através de um estudo de caso. A solução consiste em um framework de propósito geral, bibliotecas que estendem o framework para oferecer uma estratégia concreta de monitoramento, e um conjunto de recomendações e boas práticas gerais relacionando o requisito de monitoramento com todas as fases de um processo de software, com foco no Rational Unified Process $(R U P)$, a título de exemplificação, mas não se limitando a ele.

Palavras-chave: monitoramento de execução, indicador de progresso, code tangling, code scattering, crosscutting 



\section{Contents}

1 Introduction 1

1.1 Motivation 3

1.2 Overview of the Proposed Solution 3

1.3 Statement of the Contribution 5

1.4 Organization of the Dissertation 6

2 Background 9

2.1 The Ad-hoc Approach 9

$\begin{array}{lll}2.2 & \text { The Eclipse Approach } & 12\end{array}$

2.2.1 Chained Execution $\quad 15$

2.2.2 Monitor Protocol 17

$\begin{array}{ll}2.2 .3 \text { Cancelation } & 19\end{array}$

2.2.4 NullProgressMonitor 20

$\begin{array}{lll}2.2 .5 & \text { Concurrency } & 21\end{array}$

2.2.6 Limitations 22

2.3 The Aspect-Oriented Approach 24

2.4 The Metadata Approach 26

2.5 The Workflow-based Design 28

2.6 Software Performance Engineering 32

2.7 Chapter Summary 35

3 Monitoring Framework 37

3.1 Concepts and Requirements 37

3.1.1 Framework Use-Cases 41

3.2 Framework Architecture 41

$\begin{array}{lll}3.2 .1 & \text { Observer pattern } & 42\end{array}$

3.2.2 Notifications Package 44

3.2.2.1 INotificationsProvider Interface 44

3.2.2.2 Notification Abstract Class 45

3.2.3 Operations Package 46

3.2.3.1 IOperation Interface $\quad 46$

3.2.3.2 ProgressState Class 47

3.2.3.3 EOperationState Enumeration 48

3.2.4 Monitoring Package 50

3.3 Monitoring impact on Application Architecture 52

3.3.1 Sequential scenario $\quad 52$

3.3.2 Concurrent scenario 53 
3.3.3 Adapting existing systems 53

3.4 Chapter Summary $\quad 54$

4 Libraries $\quad \mathbf{5 5}$

4.1 The Service Provider Library 56

4.1.1 IDisposable Interface and using Statement 56

$\begin{array}{lll}4.1 .2 & \text { ServiceMonitor class } & 57\end{array}$

4.1.3 CustomOperation class 58

4.1.3.1 A Code Pattern for monitorable services implementation 60

4.1.3.2 Freely create and update child operations 62

4.1.3.3 Bind to another service sharing the same monitor instance 64

4.1.3.4 Bind to another service with its own monitor instance 68

4.1.3.5 Recursive Operations 70

$\begin{array}{lll}\text { 4.1.4 Notifications } & 71\end{array}$

$\begin{array}{lll}\text { 4.1.4.1 CustomOperationNotification } & 72\end{array}$

$\begin{array}{lll}\text { 4.1.4.2 GeneralNotification } & 73\end{array}$

$\begin{array}{lll}\text { 4.1.5 Service Cancelation } & 74\end{array}$

$\begin{array}{lll}\text { 4.2 The Service Consumer Library } & 75\end{array}$

4.2.1 ProgressViewer Component $\quad 75$

4.2.2 Notifications Viewer Component 76

4.3 Distributed Systems monitoring $\quad 77$

4.3.1 Service-Oriented Architecture (SOA) 77

$\begin{array}{lll}\text { 4.3.2 Events, Decoupling and SOA } & 79\end{array}$

4.4 General Purpose Versus Domain-specific implementations 82

$\begin{array}{lll}\text { 4.5 Workflow-based Extension } & 83\end{array}$

4.5.1 Workflow Activity Specification $\quad 84$

$\begin{array}{lll}4.6 & \text { Related Work comparison } & 89\end{array}$

$\begin{array}{lll}4.7 & \text { Chapter Summary } & 90\end{array}$

5 Impact on Software Processes $\quad 91$

5.1 Software Processes 91

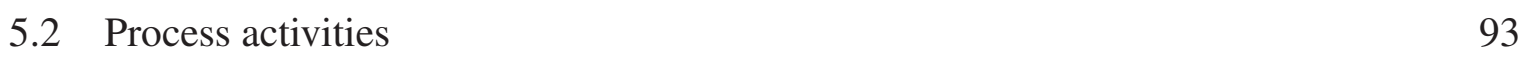

$\begin{array}{lll}\text { 5.2.1 Software specification } & 93\end{array}$

$\begin{array}{ll}\text { 5.2.2 Software design and implementation } & 94\end{array}$

$\begin{array}{lll}\text { 5.2.3 Software validation } & 95\end{array}$

5.2.4 Software evolution 96

5.3 Impact of service execution monitoring on software processes 97

$\begin{array}{llr}\text { 5.3.1 Software specification } & 98\end{array}$

$\begin{array}{ll}\text { 5.3.2 Software design and implementation } & 104\end{array}$

$\begin{array}{lll}\text { 5.3.3 Software validation } & 106\end{array}$

$\begin{array}{ll}\text { 5.3.4 Software evolution } & 108\end{array}$

$\begin{array}{lll}5.4 & \text { Chapter Summary } & 108\end{array}$ 
6 Work Evaluation $\quad 109$

6.1 Definition 109

$\begin{array}{lll}\text { 6.1.1 Context } & 109\end{array}$

$\begin{array}{lll}6.1 .2 & \text { Objectives } & 111\end{array}$

$\begin{array}{lll}6.2 & \text { Planning } & 114\end{array}$

$\begin{array}{ll}\text { 6.2.1 Determining the acceptable criteria and thresholds } & 115\end{array}$

$\begin{array}{ll}\text { 6.2.2 Experimental Setup } & 118\end{array}$

6.3 Result Analysis 118

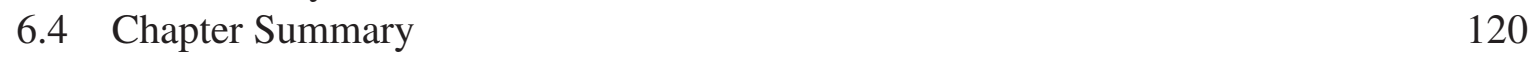

7 Conclusion $\quad 123$

7.1 Research Contribution 123

$\begin{array}{lll}7.2 & \text { Future Work } & 124\end{array}$ 



\section{List of Figures}

1.1 Progress bar example. 1

2.1 IProgressMonitor contract. 13

2.2 IProgressMonitor State Diagram. 17

2.3 Workflow example. 29

2.4 Monitorable workflow example. 30

2.5 Transformation rule for workflow initial state transition. 31

2.6 Transformation rule for activity-to-activity sequential transition. 31

2.7 Transformation rule for workflow final state transition. 31

3.1 Example of an operation tree $\quad 39$

3.2 Consumer Use-Cases 42

3.3 Provider Use-Cases $\quad 42$

3.4 Framework package diagram. 43

3.5 The Observer pattern. $\quad 43$

3.6 Notifications class diagram. 44

3.7 Example of notification tree in a user interface. 45

$\begin{array}{ll}3.8 & \text { Operations class diagram. } \\ 3.9 & 47\end{array}$

3.9 Operation state tsransition diagram. 49

3.10 The Monitoring Package. 50

3.11 Sequence diagram example for the monitoring protocol. 51

3.12 Example of typical Facade, not monitorable. 52

3.13 Facade adapted to extend IMonitorableServicesProvider. 53

3.14 Facade adapted to extend IMonitorableServicesProvider and support concurrency. 53

4.1 Dependencies between the component libraries and the framework. 56

4.2 ServiceMonitor class members. 58

4.3 CustomOperation class members. 59

4.4 CustomOperationNotification and GeneralNotification class diagram. 72

4.5 Service Consumer Library class diagram. 76

4.6 ProgressViewer component in a sample application. 76

4.7 NotificationsViewer component in a sample application. 77

4.8 Monitorable distributed service sequence diagram. 81

4.9 A general representation for a general non-monitorable Workflow Activity. $\quad 84$

4.10 MonitorableActivity Class Diagram. 86

4.11 Workflow for "Insert User" service.

5.1 Requirements engineering process [Som07]. 93 
5.2 General model of design process [Som07]. 95

5.3 General test process [Som07]. 96

5.4 System evolution [Som07]. 97

5.5 Main use-case diagram for a Course Registration System, adapted to make mon$\begin{array}{lr}\text { itoring explicit. } & 100\end{array}$

5.6 Activity diagram for the Register for Courses use-case, including execution costs. 103

6.1 Main use-cases of the project under study. 110

$\begin{array}{ll}\text { 6.2 Simplified activity diagram of the monitorable use-case. } & 112\end{array}$

6.3 Snapshot of the system while the simulation is being processed. 120

6.4 Snapshot of the system after the simulation processing has finished. 121 


\section{List of Tables}

3.1 IOperation interface methods. 47

3.2 ProgressState class members. 48

3.3 Possible states for IOperation. 49

4.1 CustomOperation class additional members. 60

4.2 Approach comparison. $\quad 89$

6.1 Analysis of previous monitorable services based on the ad-hoc approach. 117 



\section{CHAPTER 1 \\ Introduction}

Software systems have become widespread in society. Supported by the continuous emergence of improved software development technologies, processes, tools, frameworks and languages, these software systems are becoming increasingly larger and more complex [FK05]. In order to perform their aimed tasks, they often provide their end-users with a wide diversity of services, which may involve complex interactions between many different components, sub-systems, databases, and time-consuming algorithms. Therefore, as complex service implementations may lead to the execution of long-running operations, a service execution may not respond immediately after it is requested by the end-user.

It is possible that the execution of a software system service requires more processing time than originally expected by the service consumer (possibly the end-user). In other cases, the service consumer may already expect a time-consuming execution. In both cases, despite expectations, some applications display information regarding the progress of the service execution, by explicitly displaying a percentage of progress or by using graphic representations such as progress bars, as shown in Figure 1.1, along with other monitoring information such as the elapsed time, an estimate of remaining time, a brief description of the current task being performed, etc.

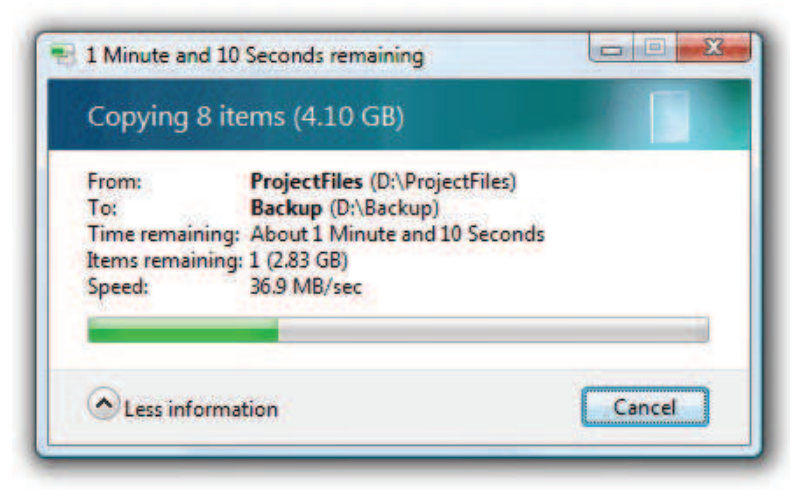

Figure 1.1 Progress bar example.

The usage of percent-done progress indicators in information systems is not new. In fact, it dates from the very first graphical user interfaces [Mil68]. Practical experience and formal experiments show that progress indicators are an important and useful user interface tool, and that they enhance the attractiveness and effectiveness of programs that incorporate them [Mye85]. Apart from the percentage itself, percent-done indicators provide the following important messages listed by Miller [Mil68]: the user knows (a) his request has been listened to, (b) his request has been accepted, $(c)$ that an interpretation of his request has been made, and $(d)$ that the system is now busy trying to provide him with an answer. 
Progress bars are particularly important for novice users since they are likely to believe that everything on the computer should operate quickly, and therefore such users are more likely to panic [Fol74] and think that the computer has crashed if it does not provide feedback while the computer is working. Progress bars are often considered a mandatory requirement for I/O-intensive services, which involve large amount of data transfer [RBR93], such as file downloading, streaming and software installations - whose progress percentage are usually deterministic, directly measured and very easy to calculate by dividing the amount of bytes already written by the total amount of bytes to be read.

Despite the immense advances on hardware platforms and computer performance in general, in the last decades, which led to better processing response times, still more recent formal experiments demonstrate that progress indicators continue to be an important graphical technique for improving human-computer interactions, with substantial impact on users' decisions to continue rather than break off software usage [FCP05], therefore explaining why percentdone progress indicators are so much widespread in current information systems.

Today, the act of displaying a graphical percent-done indicator in a user interface is often considered a task of trivial complexity, even for novice software developers and students. This is due to the immense variety of easy, ready-to-use and self-contained components that handle the calculations, scaling and perform all the painting, such as the JProgressBar Class of the Java API [API11] and the ProgressBar Class of the Microsoft .NET Framework [Net11], for example. With most of these components, all the software developer needs to do is to write code for updating the progress bar component with the current progress percentage. However, on larger and more complex software systems, this is exactly when the difficulties may arise: services execution may involve the interaction of many different components, layers and subsystems, and therefore it can require very hard work to gather detailed information upon the execution of lower-level layers, controlling the whole execution chain, and translate them all into an overall progress percentage, readily available on a higher-level layer such as a user interface progress bar.

The term "monitorable service" is used in this dissertation to denote the services that explicitly enable their consumers to extract execution information, such as the percentage of overall completion and business-specific notification messages, making it easy for them to use these information for providing on-line feedback to the end-user, registering information on systems' event $\log$ for later verification, and estimating remaining execution time. This dissertation focuses on providing a set of tools (a framework and two extended libraries), along with code patterns and process guidelines, to aid the development of monitorable services in the context of large and complex systems.

This chapter contextualizes the focus of this dissertation and starts by presenting its motivation in Section 1.1. A brief overview of the proposed solution is presented in Section 1.2. Section 1.3 presents the main contributions and, finally, Section 1.4 outlines the structure of the remainder of this dissertation.

\subsection{Motivation}

Large and complex software systems leverage companies' interest in improving software development quality and productivity [FK05]. In this context, software reuse - the process of using existing software artifacts rather than building them from scratch [Kru92] — is generally re- 
garded as the most important mechanism for performing software development more efficiently [McI68].

In fact, there is a wide variety of software components to support the display of percentdone indicators. These components often enable very effective reuse and play an important role on the user-interface part of the monitoring concern. However, despite their usefulness in facilitating renderization in the user interfaces, such components are not concerned with the difficulties involved in gathering on-line execution data and translating them into a single overall percentage. Unfortunately, both the academy and the industry lack on providing generic standards, frameworks, tools, or process guidelines specifically designed with the purpose of supporting the development of monitorable services - with few exceptions in very specific development platforms, as shown in Chapter 2.

Put simply, reuse opportunity is very limited when it comes to providing the software development community with solutions to aid the development of monitorable services. Even though the progress indicators are often present on information systems, the development strategies being adopted are often non-systematic and ad-hoc, developed from scratch and based on no standards, leading to unnecessary increase of code complexity, poor code quality, lower productivity, maintenance difficulties, among other problems detailed in Section 2.1. In fact, as explained later, these concerns only become of real matter on large systems with complex architecture, thousands of lines of code and involving many developers.

Aware of this scenario, it becomes clear that there is demand for the development of a complete solution, aiming at supporting the development of monitorable services and stimulating reuse on the software development community. This work focuses on proposing a possible solution to this demand.

The main difficulties regarding monitorable services implementation in the context of complex systems - which we address in this work - relate to (1) concern modularization; (2) code standardization; (3) development productivity; (4) increasing maintenance costs; (5) estimating and coordinating tasks and subtaks; and (6) generating accurate and uniform monitoring results.

\subsection{Overview of the Proposed Solution}

Motivated by the scenario presented in the previous sections, this work defines the concepts, requirements, design, software process guidelines and implementation of a standardized solution, aiming at facilitating the design and implementation of software with monitorable services. It focuses on minimizing code tangling and scattering [Kic99], maximizing modularization, reducing overall code complexity, providing standards and code patterns, while still freeing developers to implement business code in their preferred style, minimizing the introduction of constraints or limitations to what the developers can do - such as supporting concurrency and distribution. Therefore, it aids the software developer and architect all along the software development life-cycle.

Consequently, by reducing complexity and facilitating development, it will reduce the risks of the project and the required effort for implementing the monitorable services. The monitoring problem becomes more clear while the implementation strategy becomes systematic and predictable. Therefore, the proposed solution may possibly turn the monitoring requirement into a feasible requirement within a project with limited budget. For those reasons, this work stimulates the software developers and end-users to reconsider the monitoring requirement on 
their software projects.

In this context, we propose a solution for dealing with monitorable services implementation throughout the software development life-cycle, covering from requirements elicitation to software design, implementation and final delivery to the end-user environment. Implementing monitorable services involves dealing with five main concerns:

- Specification. Monitorable services should be clearly identified early in the software development life-cycle, ideally on requirements specification stage, and the involved workflows should be clearly described. This concern is covered in Chapter 5.

- Architecture design. Software architecture should facilitate monitoring information to be exchanged between the system components. This concern is covered in Chapters 3 and 4.

- Monitoring aggregation and implementation. The developers need to add code for generating monitoring information and provide feedback during services' execution. This concern is the focus of this work and is covered in chapters 3 and 4.

- Feedback displaying in user interface. Once the monitoring feedback is received, it is often displayed using graphic percent-done indicators. Displaying these indicators is often a trivial task supported by most development platforms.

- Validation and tuning. During the test phase of software development, the generated monitoring information should be validated towards uniformity and consistency and the monitoring implementation should be tuned in order to optimize quality. This subject is covered in Chapter 5.

The proposed solution is mainly focused on the design and implementation phase and consists of three different components:

- Framework. A general purpose framework that does not establish any specific strategy for monitoring, but defines core concepts, design patterns and abstract class implementations for supporting execution monitoring, without making assumptions on the nature of the software being developed.

- Libraries. Two libraries extending the framework, implementing a specific strategy for monitoring, one for usage of the service consumer and the other for usage of the service provider.

- Software process. Recommendations and guidelines for considering the monitoring requirement in the wider context of a software process.

\subsection{Statement of the Contribution}

As a result of the work presented in this dissertation, a list of contributions may be enumerated:

- A characterization of the execution monitoring problem. It was conducted a study about the monitoring problem in order to identify current and possible approaches for implementation and the main difficulties involved. 
- A solution to support monitorable services implementation. It specifies the requirements, design and implementation definition for the framework and extended libraries that support the development of monitorable services.

- Guidelines for adapting Software Processes. It presents guidelines for the software developers and designers to consider in all phases of the software development life-cycle.

- Case study in the proposed solution. It presents a case study performed in a private software development organization and shows significant results towards the validation of the solution.

The proposed solution distinguishes from other existing related solutions in many aspects which have been detailed throughout the dissertation. Notably, we can point out its general purpose principle (an abstract definition that enables extension for different monitoring strategy implementations), platform-independent design, more compact and regular code patterns, facilities for automatically binding tasks and subtasks based on the call stack, seamless concurrency support, typed message tree (monitoring data is not limited to a single percentage), complete state diagram (for automatically handling of unexpected errors and explicitly representing intermediate cancelation states), maximization of decoupling between business code and monitoring-related code, ability to turn a non-monitorable service into a monitorable one without the need to refactor the existing service consumers (monitoring does not affect method signatures), among others.

Since it focuses on providing a general purpose solution to aid the development of any software based on the object-oriented paradigm, it leaves space for improvements and for other related work that is not directly addressed by this work, listed as follows:

- Full development automation. The proposed solution does not completely automate the software development process. Instead, it introduces a generic abstract framework and focus on the patterns for manually delimiting monitorable operations within the code. A fully automated strategy possibly exists for a given domain-specific context — although we doubt there exists single optimal general purpose strategy that serves any context without introducing assumptions or limitations to the development style. If such an optimal strategy exists, it would be certainly implemented as an extension of our proposed solution. This is possible because we have decoupled the general purpose concepts from the actual strategy for defining and generating monitoring information: the first is encapsulated within the framework; the latter is handled by the proposed libraries which are extensions of the general purpose framework.

- Full monitoring concern modularization. One of the main challenges in the monitoring problem is related to the modularization of the monitoring code, aiming at the minimization of monitoring code tangling and scattering [Kic99] throughout the business code. Although the proposed solution provides significant reduction of code tangling and scattering, as shown in Chapter 6, it does not completely eliminate it. An optimal implementation strategy that completely eliminates code tangling and scattering possibly exists, and it could be certainly implemented as a new extension of the proposed general purpose framework. 
- Specific monitoring strategies' implementations. As the solution focuses on introducing monitoring main concepts and a general purpose strategy based on weights assignment, this strategy may not be optimal for some specific scenarios. It is out of the scope of this dissertation to elaborate different monitoring strategies, but to provide the background needed to extend the framework and implement any other monitoring strategy.

- Software process specification. We do not propose or derive any specific software process specification that considers the monitoring problem. Instead, we provide some guidelines and generic guidance regarding general software processes. These guidelines are certainly applicable to any software process. To illustrate, we provide some examples based on the Rational Unified Process (RUP).

- Evaluating the importance of the monitoring requirement. It is assumed that the monitoring requirement is actually considered important to the software development, or at least desirable. It is out of the scope of this work to discuss whether or not the monitoring actually provides cost-effective benefits. In addition to the observable industry practices, some experiments and academic researches have already demonstrated the benefits of the monitoring requirement in improving the human-computer interaction [Mil68, Fol74, FCP05].

\subsection{Organization of the Dissertation}

This dissertation is organized in other six chapters described as follows:

- Chapter 2 presents background information regarding the problem of implementing monitorable services. Existing approaches for implementing such services are reviewed and analyzed concerning their aids, limitations and characteristics. Some related topics that help on understanding the monitoring problem, such as Software Performance Engineering, Aspect-oriented Programming and Workflows Modeling, are also reviewed. Existing difficulties and problems related to the implementation of monitorable services are systematically enumerated and discussed.

- Chapter 3 describes the concepts that form the foundation of the proposed framework, as well as the proposed framework itself. The requirements, design and implementation aspects are discussed in details. Examples on how to implement monitorable services are also demonstrated, by using the framework elements.

- Chapter 4 continues the previous chapter by describing a pair of software libraries that extend the general purpose framework aiming at implementing a concrete monitoring strategy and therefore providing additional facilities for the development of monitorable services.

- Chapter 5 discusses the impact of considering the execution monitoring requirement on general Software Processes, and analyzes how it can affect the software development life-cycle. More specifically, it analyzes each phase and activity of the Rational Unified Process and establishes good-practices, guidelines and standards for considering the monitoring requirement throughout the development process. 
- Chapter 6 reports on the conclusions drawn upon the evaluation of the proposed solution with respect to their effectiveness towards increasing productivity, code quality and monitoring quality.

- Chapter 7 concludes this dissertation by summarizing the findings, proposing future enhancements and ideas of related work, along with some concluding remarks on the execution monitoring problem. 



\section{CHAPTER 2}

\section{Background}

This chapter presents some existing approaches towards the implementation of execution progress monitoring capabilities. First, we discuss an ad-hoc approach to implement monitoring requirements and present common problems challenged by programmers without support of frameworks, patterns, tools or standards. We also present the facilities provided by the Eclipse API [Ec106c] to support progress monitoring, show how it works and summarize the benefits and drawbacks of its usage. Then, we provide a brief discussion on how an Aspect-oriented Approach could be used to improve code modularity. In addition, we discuss how a Metadata Approach can deal with the monitoring concern. Furthermore, we provide an overview of Workflow-based design and how its nature fits for supporting service execution monitoring. Finally, we discuss how the Software Performance Engineering discipline techniques also relate to this work to some extent.

\subsection{The Ad-hoc Approach}

In most engineering disciplines, systems construction is based on the composition of existing components and subsystems, which have already been tested in different environments and proven to work [Som07]. Software engineering is not different. Many paradigms, language constructs, design patterns, tools and frameworks have been developed over the years to improve software reuse capabilities. The benefits of software reuse goes beyond productivity. It enables organizations to meaningly improve absolute software quality, increasing modularity, lowering software complexity and code redundancy and thus reducing the occurrence of bugs [PM07].

In order to reach a high level of software reuse, systems architects need to understand all the software requirements in the early development stage. This is crucial for the identification of reuse possibilities and for guiding system architecture, design and chosen technologies to enable third-party components reuse.

In our case, services execution progress monitoring must be a very clear application requirement for the system architect. Otherwise, ignoring this requirement may lead to an architecture design that does not allow a reusable solution to the problem, which may lead to code rewriting and increase of software complexity. As a general rule, the larger the system, the greater the code redundancy and complexity [PM07]. Chapter 5 provides details on the impact of supporting service execution monitoring in the Requirements development phase and in the Software Development Process as a whole.

In this work, the term "ad-hoc approach" is used to designate the process of implementing monitorable services without the systematic adoption of reuse. In this case, no standards, patterns, frameworks, tools, APIs or process guidelines — specially conceived and designed to consider the monitoring requirement — are used to support the software development. In such 
case, the software developer will have no specific patterns to base his implementation. Therefore, the implementation will be based on a few on-demand decisions that may work in that specific context but would require code rewriting if the core business code is changed.

On simple systems — no distribution, few layers and short call stacks —, or in a situation where the system under consideration is already developed and the monitoring requirement is needed just for one or two services with low complexity and implemented as a few lines of code, it may be more practical and productive to adopt the ad-hoc approach, not worrying about reusing any tools, frameworks or patterns and simply solving the monitoring problem locally and focused on the specific service implementation context (in such cases, implementing the monitoring requirement from scratch can require less effort than searching and understanding general purpose standards).

However, the adoption of the ad-hoc approach is often unconscious. Software designers focus on business and functional requirements and simply do not realize the crosscutting [Kic99] nature of monitoring requirement and its potential for increasing code complexity. When the monitoring requirement is not given proper consideration in an early stage of software development, it may result in a design and architecture that does not favor monitoring implementation. In addition, there are too few tools and patterns specifically designed to support monitorable services implementation, on both industry and academy. Therefore, even when the software developers identify the need of a framework to support the requirement implementation, since they will simply find no reuse opportunity, there is a chance they resist to implement such a framework from scratch, specially if the monitoring requirement is not considered essential when compared to other functional and business requirements or under limited budget and schedule circumstances, deviating from the core business focus.

The Listing 2.1 shows a fictitious example code — for didactical purposes — that updates a graphical percent-done indicator in a user interface.

Listing 2.1 Sample code updating progress bar

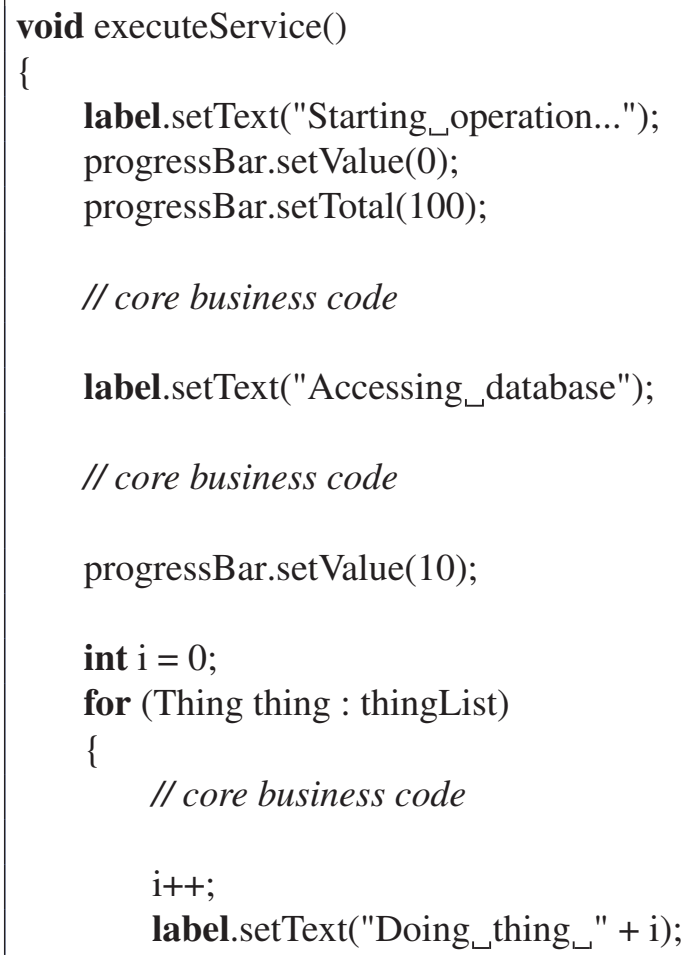




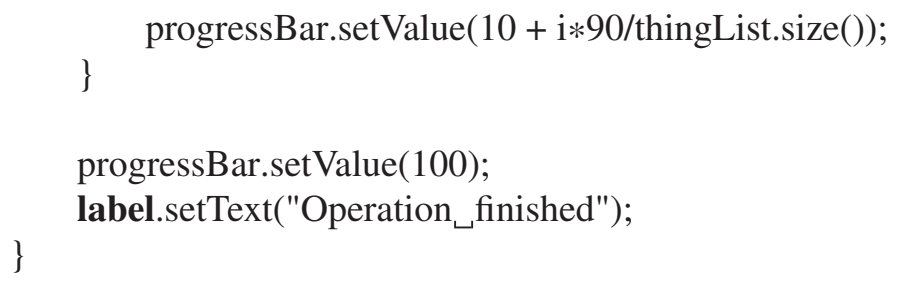

The first three lines of code display a message to the end-user and clear the progress bar component, ensuring it to be at an initial state of zero-percent, before the actual service processing begins. The next two lines of code represent an initial preparation for the service execution. Since it is supposed to take ten percent of the overall service execution, the progress bar component is updated to reflect this percentage. The next lines of code represent the main service processing, which is based on the iteration through a list of elements. Each element is processed, one by one, and the progress bar component is updated in order to represent the current progress state. In this case, the elements are supposed to take a constant processing time, so that the progress bar value can be updated with a simple proportion based on the number of elements already processed and the total number of elements in the list.

This kind of code can meaningly increase in complexity, if operations involve chained execution, concurrency, user cancelation handling, error handling, and so on. The problems of such ad-hoc implementations can be summarized as follows:

- Core business decoupling. It does not allow for decoupling of core business and execution monitoring code. The amount of lines of code implementing execution monitoring can be even greater than the lines of code implementing the core business. Business code becomes scattered and interlaces monitoring code. Notice that the code sample shown in Listing 2.1 is fictitious and does only contain monitoring-related code, whereas possible business-specific code location is pointed out as comment lines.

- Support for layered and modularized architectures. Large systems often implement their business-specific rules on a multi-layer and multi-component architecture. System architects are naturally stimulated to do so due to the many benefits involved. In fact, modularity became a major practice in the software market [PM07]. The ad-hoc approach does not deal well with modularized business code, for it cannot provide progress details of services performed indirectly by lower-level components and submodules. The developer would need to modify each component, one by one, by adding specific monitoring code to update shared global-scope variables for updating the overall progress state. In practice, an ad-hoc approach can lead lower-level layers to "know" higher-level layer variables and thus violate compliance with a basic rule of layered pattern: service providers should make no assumptions upon the service consumer nature.

- Limits reuse capabilities. Without making use of code patterns, frameworks or tools for supporting execution monitoring, code becomes replicated among distinct monitorable service implementation, which increases overall code maintenance difficulty as the service requirements change.

An additional difficulty for the ad-hoc approach is that, for reasons beyond this work context, most User Interface technologies require User Interface Components to be accessed and 
updated from within a single thread [Se102, HM00]. This limitation provides additional complexity for long-running business code, as they should run on separate threads and still synchronize progress updates from a different thread. The example in Listing 2.1 shows a sequential implementation, with the business-related code tangled to the monitoring-related code. Let us suppose that the loop iteration needs to run on a different execution thread, but inside the loop there is code for updating the progress bar (a UI operation). In such case, the progress bar should not be accessed directly from the business thread, because most UI platforms only allow a single thread to directly access and update the UI components. Dealing with such case would require additional code in order to enable cross-thread method invokations.

For the reasons exposed, we conclude that monitoring code will often require the support of patterns, tools, frameworks or native language constructs to support the implementation and avoid unnecessary increase of code complexity and scattering among the main business code, while a non-systematic approach will rarely handle the monitoring problem in a proper manner, except for very simple scenarios such as the one shown in Listing 2.1.

\subsection{The Eclipse Approach}

The Eclipse API is a feature-rich component library providing the Java Development Community with a variety of tools for software development common use - such as UI Components, concurrency control, data access, I/O control, etc. In addition to this set of features, it provides some facilities to the implementation of progress execution feedback to the end-user.

The Eclipse API approach relies on the concept of Progress Monitors. A Progress Monitor is a callback interface that allows a long-running task to report execution progress and respond to cancelation request. Typically, a UI Component (the consumer) will create a monitor instance and pass it to a low-level component (the long-running service provider) that does not know or care about the UI. Thus, this callback interface is an abstraction that allows for decoupling of $\mathrm{UI}$ and non-UI components [Ecl06b]. The IProgressMonitor contract is shown in Figure 2.1 [Doc10].

\begin{tabular}{l}
\hline \multicolumn{1}{c|}{\begin{tabular}{c}
\multicolumn{1}{c|}{ «interface» } \\
IProgressMonitor
\end{tabular}} \\
\hline +beginTask(in name: String, in totalWork: int) \\
+done() \\
+isCanceled() : bool \\
+setCanceled() \\
+setTaskName(in name : String) \\
+subTask(in name: String) \\
+worked(in work: int) \\
\hline
\end{tabular}

Figure 2.1 IProgressMonitor contract.

The IProgressMonitor interface is a variation of the Observer pattern, as it does not declare a subject for event registration (attach and detach). The class implementing the interface is the entity actually interested in receiving the event and thus there is no intermediate entity between the sender and receiver of events. 
Any monitorable service implementation needs to receive an IProgressMonitor as parameter in order to send progress feedback data. During the monitorable service execution, the service provider needs to properly invoke the monitor interface methods in order to report current execution state. All the interface methods are intended for the service provider to actively invoke. As an effect of each method call, the service consumer (or a third listener entity interested in receiving progress feedback) becomes aware of a new progress state update being reported. Therefore, the service provider is absolutely active - as it makes all the method invocations and is responsible for reporting progress state updates - , while the service consumer (or a third entity) is absolutely passive - as it does nothing but receive the progress reports. Notice that the entity actually implementing the IProgressMonitor interface could be anything, such as the service consumer itself, or a UI component such as percent-done indicator, or an intermediate proxy redirecting the reports to multiple entities, or even a logger registering the monitoring reports for future analysis and tracing.

Each monitor instance must comply with a strictly defined lifecycle [Ec106b]. Before we detail its lifecycle, let us briefly introduce the methods that compose the IProgressMonitor interface:

- beginTask. It is invoked in order to report that the service actual activity is about to begin. It is parametrized by string representing a brief description of whatever the service is supposed to do, and by an integer representing the total number of work units to be performed.

- done. It is invoked in order to report that the service execution has ended without being canceled.

- isCanceled. It is invoked meanwhile during the service execution in order to detect whether a cancelation request has been performed.

- setCanceled. It is invoked after the cancelation request is detected and properly handled, in order to report that the service execution has ended and the cancelation request has been acknowledged.

- setTaskName. It is invoked during the service execution in order to change the main description originally passed to the beginTask method.

- subTask. It is invoked during the service execution in order to report a more detailed description of the current operation being performed.

- worked. It is invoked during the service execution in order to report that a certain number of units of work has just been performed.

Therefore, the monitor lifecycle can be summarized as follows:

- The first method that must be invoked is beginTask. For the parameter representing the number of "units of work", the developer must give an estimate for the amount of work needed for the task to complete. 
- After beginTask is invoked, current progress status must be reported by calling worked or subtask. The sum of values passed to the worked method must equal the total work passed to beginTask. The subTask method can be called as often as desired in order to report which part of the task is currently executing.

- If a cancelation request is performed, after beginTask is invoked, and the request is detected during the service execution by invoking isCanceled, then the service provider needs to handle the cancelation. After the cancelation request is handled, it must invoke setCanceled in order to report that the service execution has finished and the monitor is currently in a canceled state.

- Finally, as the task completes without being canceled, the method done must be invoked in order to explicitly indicate the higher-level component (possibly the end-user) that all the work has just completed. To make sure that it will get called, it is usually placed inside a finally block at the very end of the operation, a code pattern for implementing monitorable services [Ec106b].

Listing 2.2 shows how the code of a long-running operation might look like:

Listing 2.2 Long-running operation example code

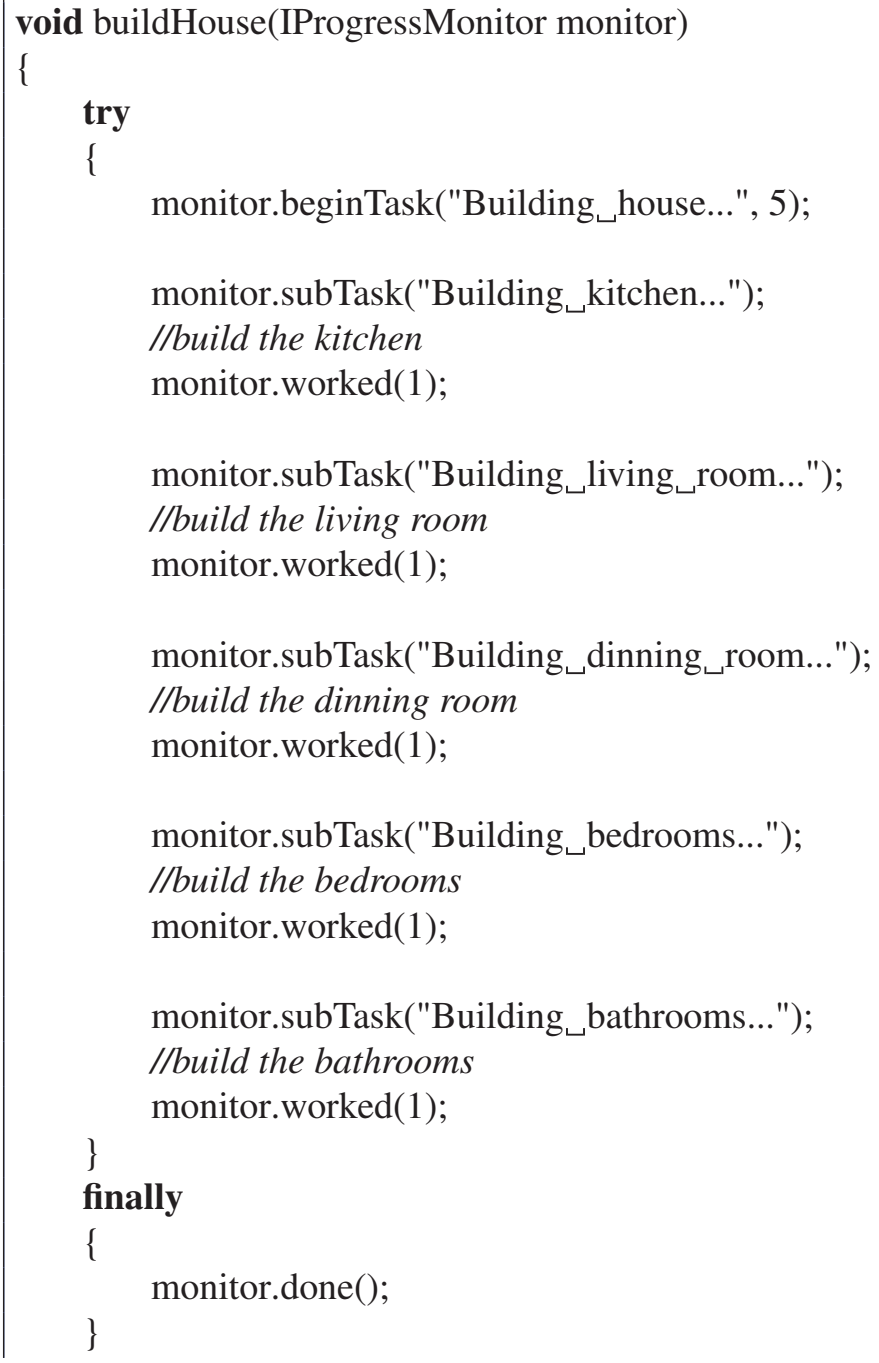


Since the interactions between the service provider and the monitor instance are synchronous and rely on calling methods in the IProgressMonitor interface to report current state, the listener - anyone responding to those method calls, such as the service consumer or a percent-done indicator in the user interface - should provide a fast and robust implementation. If the entity which is actually implementing the IProgressMonitor interface delayed on handling the on-line progress reports (such as working on user interface renderization, performing thread blocking operations, or throwing uncaught exceptions), the service execution performance would be meaningly degraded as an effect of the monitoring reports. In order to deal with such a situation, one could implement the monitor interface by queueing the reported progress updates and rapidly returning the method calls to the service provider execution, with no immediate processing of the progress updates, which could be delegated to a separate thread consuming the queue [Doc10].

\subsubsection{Chained Execution}

Long-running operations often reuse other operations as part of their implementation. In a typical object-oriented architecture, methods that represent higher-level services have their implementation based on a deep method invocation chain where each called method represent a lower-level service. Similarly, in service-oriented architectures, services are expected to be built on top of other services, as an aggregation of lower-level services [MPL07].

The Eclipse API provides a specific type of monitor that attaches to the main monitor and works as a subtask of the main task.

When using progress monitors in Eclipse, an important rule is that all monitorable methods expect an unused progress monitor. These methods are not designed to receive a monitor whose method beginTask has been previously invoked or a monitor that has already registered some units of work. The reasons for this are clear: methods can be called from a variety of places and cannot predict how many units of work they represent in the context of a long-running operation. An API method that deletes a file might represent all the work for an operation or might be called as a small part of a much larger operation. Only the code at the top of the call chain has contextual knowledge for estimating how many units of work the file deletion will take in proportion to the rest of the task [Ec106a].

The class SubProgressMonitor acts as a bridge between caller and callee. A new SubProgressMonitor instance should be created every time a chained service is executed as a chained subtask of the main monitor. This monitor knows the amount of work units the caller has allocated for the subtask in the higher-level context and also knows the total amount of work units allocated for the subtask in the lower-level context as the method beginTask is called. Thus, when the child task in the lower-level context reports progress feedback by calling worked on the SubProgressMonitor, it proportionally scales the amount of work to the higher-level context and updates the main task progress state.

To put it simple, let us have a look at a fictitious example. Suppose we are implementing a transfer method by calling credit and debit methods on the involved accounts. Suppose we estimate credit to represent $60 \%$ of work and debit to represent $40 \%$ of work.

Listing 2.3 Chained operation example code 


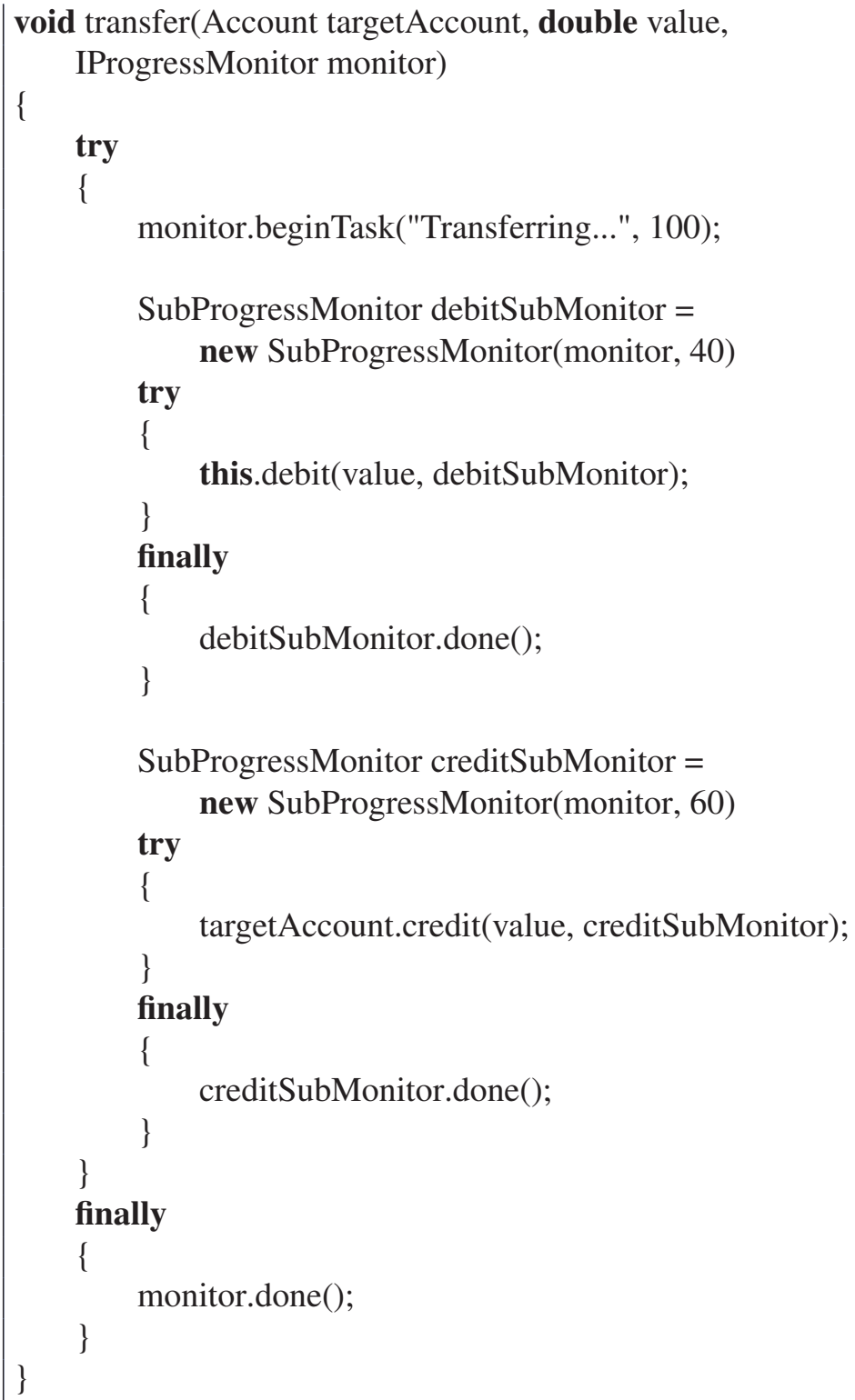

\subsubsection{Monitor Protocol}

Although it is not made explicit in the contract, any monitor can be in exactly four states. Any given implementation may or may not track those state changes and may or may not do anything about them, but still it is important for the monitorable method developer to understand those states, as he cannot make assumptions about the monitor implementation. The states are:

- Pristine. The initial state of a newly created instance of IProgressMonitor. This state indicates that the process has not started yet. Thus, in a higher-level context, the consumer still waits for the lower-level process to execute.

- In Use. The state of the monitor after the call to beginTask. It must be called only once. There is a useful code pattern to deal with this obligation. It will be shown below. 
- Canceled. The state of the monitor after call to setCanceled from In Use state. This method should only be called once and should always be called on a monitor when beginTask has already been called.

- Finished. The state of the monitor after the call to done from In Use state. This method should only be called once and should always be called on a monitor when beginTask has already been called.

Figure 2.2 shows the IProgressMonitor states transition diagram.

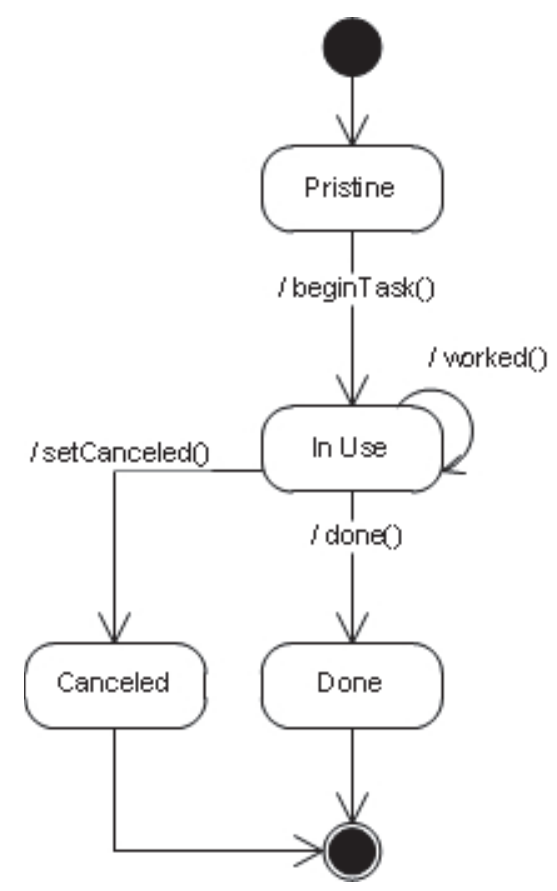

Figure 2.2 IProgressMonitor State Diagram.

One might notice that not all the monitor interface methods appear in the state transition diagram shown in Figure $2.2-$ isCanceled, setTaskName and subTask were suppressed. The suppressed methods are not mandatory in the monitor lifecycle, since they play a secondary role in the monitor protocol. In addition, they do not trigger any state transition. Therefore, these methods do not really affect the monitor lifecycle, and serve only to allow the service provider to exchange additional (secondary) information during the service execution (they are all invoked during the In Use state).

In order for this approach to work properly (with reasonable consistency and uniformity), it requires the developer to understand the interface protocol and follow a few rules as detailed in the sequel [Ec106c]. Each rule is described as a separate paragraph.

- Each monitorable method must receive its own monitor as a parameter. All interaction with a progress monitor is based on the interface IProgressMonitor and it defines the protocol behavior expected. The contract implementation is provided by a higherlevel context and the code of a monitorable method should not make any assumptions about its implementation, neither about what has been processed previously or supposedly would get processed next. Although the monitor implementation and behavior is a 
higher-level responsibility, the monitorable method code is responsible for controlling its state changes and calling its methods in a proper and reasonable order, according to the contract protocol.

- Ensure to always start and finish the monitor. The reason for this is that the monitorable method code is not able to know if it is being called as a main task or as a subtask of a broader task. Therefore, if it does not complete its monitor, the parent task might end up with an incorrect count for its own task. There is a code pattern that can be used for respecting this rule, as shown in Listing 2.4. If the method execution flow throws an unhandled exception, the finally block will still execute and the monitor will remain on a consistent state.

Listing 2.4 Code pattern for monitorable methods

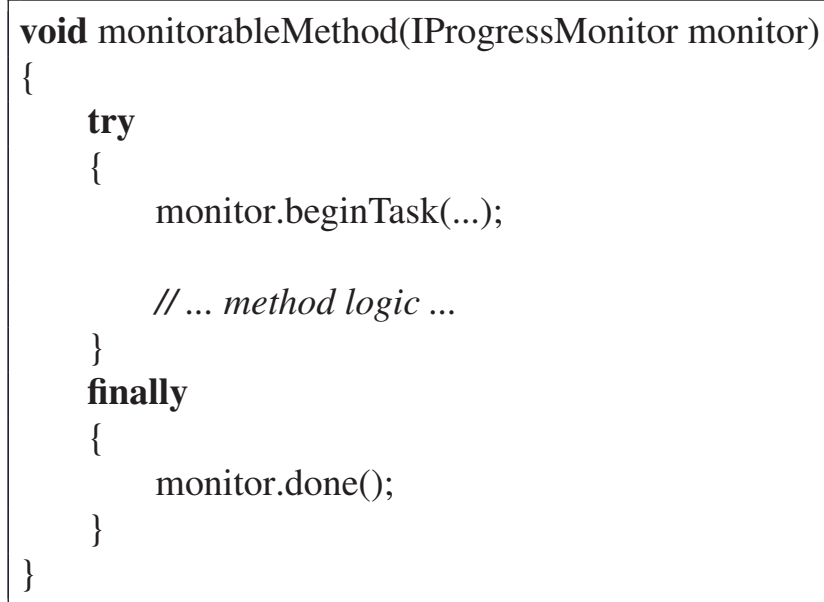

- Do not reuse the same monitor instance. Sharing a monitor instance is almost always undesirable, unless the code knows exactly what the implications are. This is due to the fact that the monitor must be in Pristine state when the method beginTask is called. The only time a single instance of a monitor passed to a certain piece of code can be reused in multiple places is when the code in such methods is so intimately coupled that they in fact constitute a single try/finally block. Also, for this to work, each method must know exactly who does beginTask/done calls, and also how many work items they represent of the total reported to beginTask so that they can make the correct reports. It is generally more trouble than it is worth. Thus, it is better to follow the regular pattern of one exclusive monitor instance per method and the code as a whole is more maintainable [Lin09].

- Do not guess the total number of work items. Only clearly deterministic code can have progress monitoring capabilities. In the beginning of the method implementation, as it calls beginTask, it must provide the total number of work items that is going to be executed. There is no rule for the semantics given to the work items, for it may depend on the business logic and varies from method to method, but that number of work items passed in beginTask must represent the exact sum of the items processed during all method execution, passed through method beginTask. This rule is due to the fact that the percent-done progress feedback given to the higher-level context is based on those 
numbers. There are typically two basic patterns where the developer is able to know how many items he want to process: either he is going to call several different methods to achieve the full result, or he is going to call one method for each instance in a collection of some sort. Either way he knows the total item count to process: the number of methods or the size of a collection. Variations of this are obviously combinations of these basic patterns. Thus, the developer just needs to add code for multiplying and summing it all up before calling beginTask.

\subsubsection{Cancelation}

The monitor also makes it possible to respond to cancelation requests. When the user requests cancelation, the method isCanceled will return true. A long-running operation implementation should check this value occasionally and abort if a cancelation has occurred. A common method of quickly aborting a long-running operation is to throw the specific exception OperationCanceledException. Alternatively, one handle the cancelation requests by explicitly skipping the algorithm execution flow with conditional probes.

The sample code in Listing 2.5 shows an example of how execution cancelation requests can be handled.

Listing 2.5 Cancelation Handling

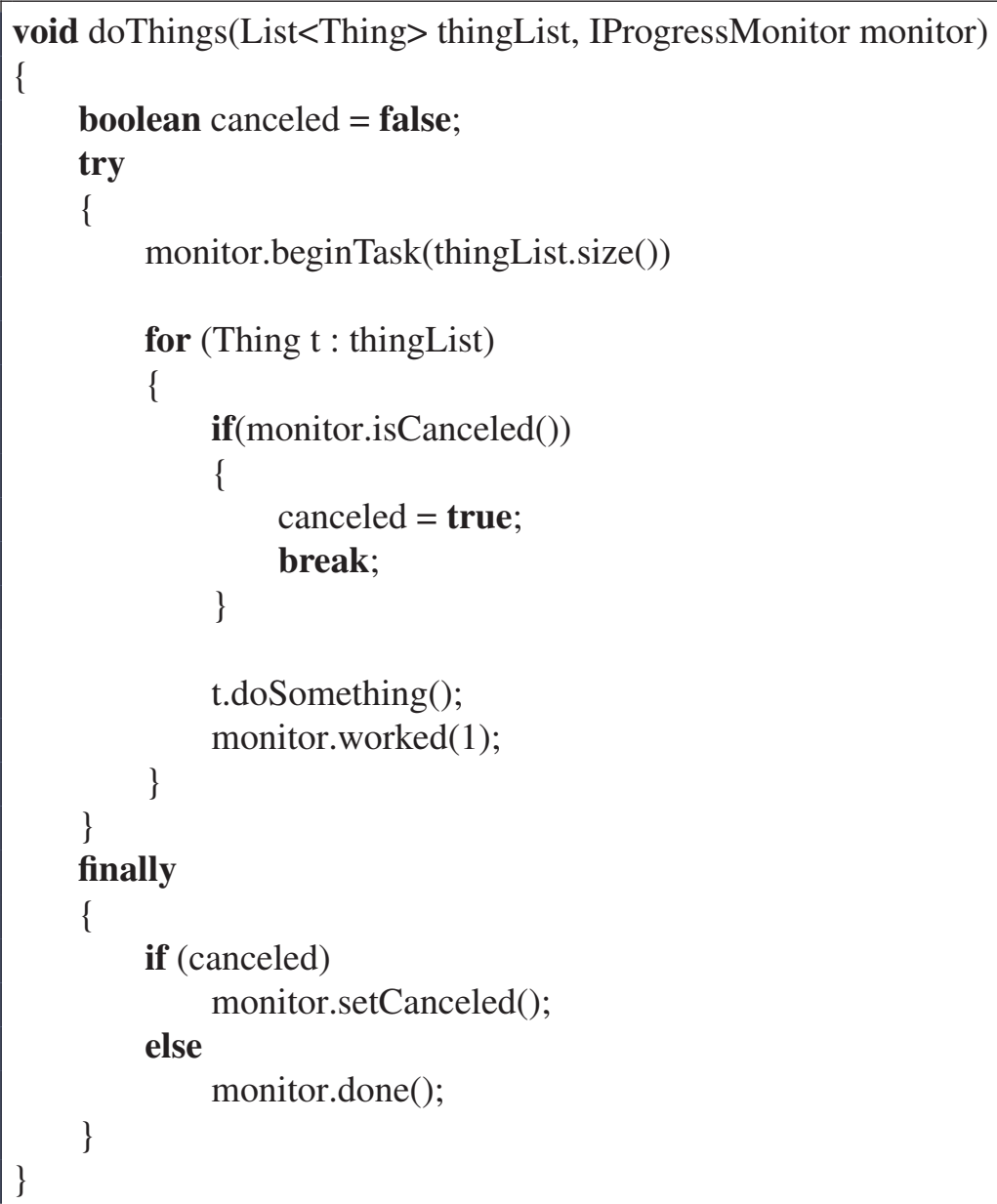

Once the cancelation request is detected by the monitorable service, by calling isCanceled, 
it interrupts on-going execution by breaking the loop and sets a local variable to indicate that a cancelation has been handled. The beginTask/done code pattern is then adapted to consider the cancelation case. One could inquire whether the local variable is necessary, by considering calling isCanceled once again inside the finally block. The problem with this approach is that isCanceled returns whether there is a cancelation request, but it would not guarantee that the cancelation has been properly handled while the method executed.

\subsubsection{NullProgressMonitor}

It is possible for a higher-level context to call a monitorable method with no interest in monitoring its execution progress. For example, this situation can occur on computer-to-computer interactions or autonomous background jobs running on application servers, when there is no actual end-user interested in receiving progress feedback regarding the task under execution, since the service execution is not directly originated from a user explicit request. In such cases, the service consumer may pass a null reference of IProgressMonitor to this lower-level method. Hence, the monitorable method would need to consider the possibility of receiving a null reference of the monitor in every single point of code providing monitoring feedback. In order to avoid the need to consider null reference all along the code, the Eclipse API provides a native special implementation of IProgressMonitor, the NullProgressMonitor class, along with a simple code pattern to deal with this in the monitorable method implementation, to avoid worrying about null references when working with the monitor passed as parameter.

The sample code in Listing 2.6 shows an example of how these null references can be handled.

Listing 2.6 Cancelation Handling

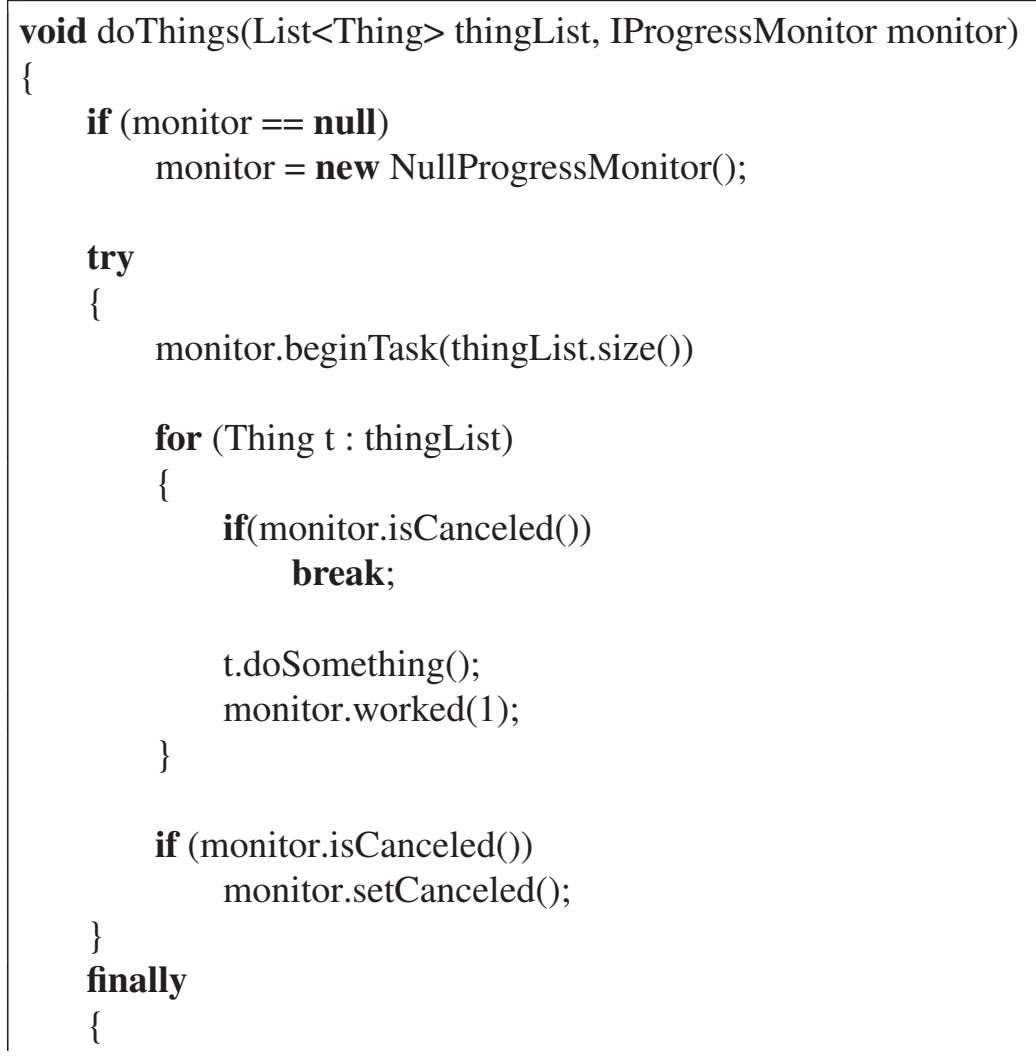




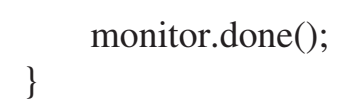

\subsubsection{Concurrency}

The IProgressMonitor interface described in previous sections provides the ability to execute subtasks that seamlessly update progress of parent tasks. The code patterns and rules presented for handling those subtasks and interacting with the monitor rely on the assumption that all code runs sequentially, within a single thread. Although this assumption holds in some cases, service implementations can involve concurrent execution of subtasks, and it would be a very critical limitation not handle those methods. In fact, the contract protocol in IProgressMonitor supports concurrent running subtasks, with different SubMonitor instances updating progress concurrently, but it is a developer's responsibility to deal with thread manipulation and controlling different instances of SubProgressMonitor executing concurrently.

As an additional concern, when dealing with concurrent subtasks, the developer must be careful not to call done while there are still running subtasks. Therefore, elsewhere, the parent task thread should wait for the subtasks to complete their monitors before finishing the parent task monitor. The example in Listing 2.7 adapts the example shown in Listing 2.5 to illustrate this.

Listing 2.7 Dealing with concurrency

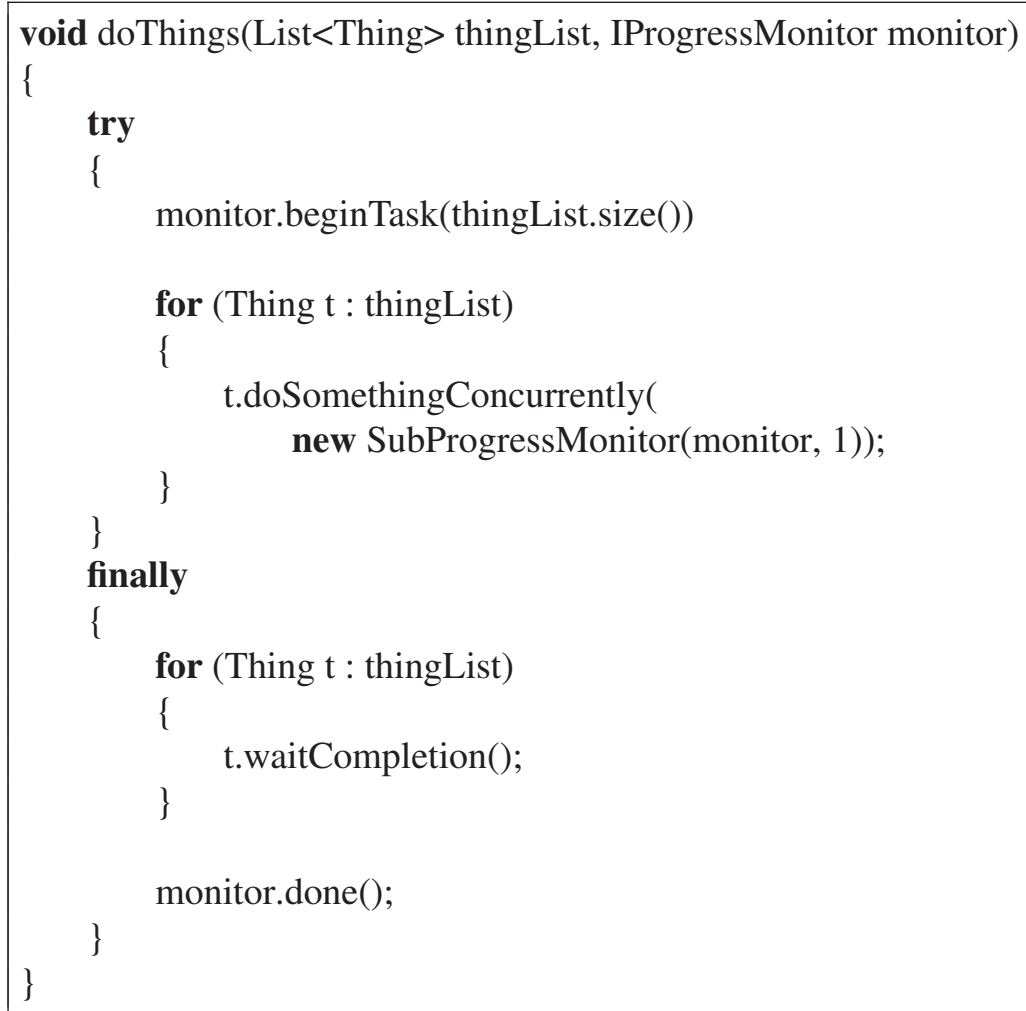

In the sample code 2.7 , notice that the developer has to explicitly add code to wait for the other threads to complete before calling done and thus indicate to the higher-level context that the main operation as whole has been completed. 


\subsubsection{Limitations}

The Eclipse approach provides some facilities for implementing monitorable services that provide progress feedback and it is certainly a better approach than the ad-hoc (which is actually not systematic at all). Despite its benefits, the Eclipse API approach has some limitations and drawbacks that architects and developers should consider before deciding to use it as a monitoring pattern for developing their applications:

- It degrades business code legibility. Creating and finishing tasks, calculating total work items, creating try/finally blocks, verifying cancelation state and updating task worked items requires some lines of code that have nothing to do with the core business implementation. These lines make the code more difficult to understand and maintain. It is particularly difficult to maintain the number of work items used to update execution progress; if the code gets changed by different programmers, it is critical that every programmer involved understands the semantics behind the work items.

- It affects method signatures. Every monitorable method must receive its own progress monitor. This rule is very "architecture-intrusive", as it forces changes to method specifications by adding a parameter that does not directly relate to the core business implementation, increasing code complexity and reducing understandability.

- It does not support distributed service monitoring. The Eclipse Approach strategy is based on an interface callback which is passed by reference to the service provider. In most distributed service-oriented architectures, callbacks and object references are simply not supported due to the cross-platform difficulties that typically occurs on distributed scenarios.

- It is not consumed seamlessly. In the Eclipse approach, the consumer creates the monitor instance. This is based on the assumption that there are only two actors in the execution process: the caller (service consumer) and the callee (service provider). The caller is obviously interested in the service execution, but it is not necessarily interested in monitoring the progress execution. A different approach could consider distinct actors for the service consumer and monitoring observer. The service consumer could execute the service without even realizing there could be a different actor observing the execution progress and receiving progress feedback.

- It requires developer to explicitly bind tasks and subtasks. The majority of the code implementation for a service runs in sequence, not concurrently. Thus, if a new task is created while the previous one is not yet finished, in a non-concurrent environment, the new task is obviously a subtask of the previous one. The API does not track these relations automatically and the programmer has to explicitly link the subtasks to the main monitor. A better approach would automatically create these bindings based on the call stack, leaving less effort to the developer and reducing code complexity.

- It does not provide message hierarchy and typing. The API is focused on progress state which relies on a number of work items. This is enough for percent-done progress indicators (such as progress bars). On the other hand, it is not fully featured for handling additional task messages bound to the task execution, such as warnings, error messages 
and business-specific information that might be useful in the higher-level context. It provides the subTask method, which enables the monitorable method to send messages, but they are not strongly typed messages and they do not have an explicit hierarchy inside the task. For example: messages, submessages, warnings inside messages, etc. Messages are flattened and untyped.

- It does not track tasks finished with errors. Almost every service implementation has fault or error conditions which may lead to operation abrupt termination, such as raising unexpected exceptions or business-specific validation. In these cases, the finally block will execute and the done method will be called on the monitor, just as if the operation had succeeded normally. The monitor protocol does not distinguish the tasks completed with errors from those completed with success, neither automatically or manually. A better approach would track erroneous tasks with specific state for representing error situations.

- It depends on work items estimates. Progress feedback reporting is based on calls to worked method and the percentage of the task execution is calculated dividing the number of work items done by the total number of work items estimated in the beginning of the task execution. There is no automation. The programmer needs to reasonably distribute work items among the calls to the worked method so that the progress execution is reported uniformly along the whole task execution.

The framework presented in Chapter 3, along with the library presented in Chapter 4, addresses most of those limitations and minimizes the impact of others. Furthermore, we will also discuss inherent difficulties of monitoring services execution.

\subsection{The Aspect-Oriented Approach}

In a pure object-oriented approach, such as the one shown in Section 2.2, we can create classes to encapsulate state transitions, provide methods for increasing the cost, notify consumer as execution progress changes, etc. An object-oriented approach allows the developer to encapsulate complexity and reuse the same logic, but it still lacks when it comes to code modularity, leading to code scattering and tangling [Kic99].

According to [Kic99], object-oriented programming does not completely avoid code scattering and tangling for the implementation of crosscutting concerns, despite the efforts for the use of a good design and recommended patterns.

Code scattering clearly occurs in the monitoring context, as the developer has to add similar code instructions in different modules and in distinct classes of the application to deal with the monitoring concern. In addition, business code is also tangled with code that deals with the monitoring concern. Thus, by using object-oriented techniques, despite the quality of design, the code that handles monitoring concern will not be concentrated on a single entity, due to the inherent crosscutting nature of the execution monitoring concern.

The aspect-oriented paradigm provides additional language concepts that better represents crosscutting concerns. We could adapt the object-oriented solution presented in Listing 2.6 by defining a single aspect that affects every monitorable method. The aspect should look like that presented in Listing 2.8: 
Listing 2.8 Monitorable methods aspect

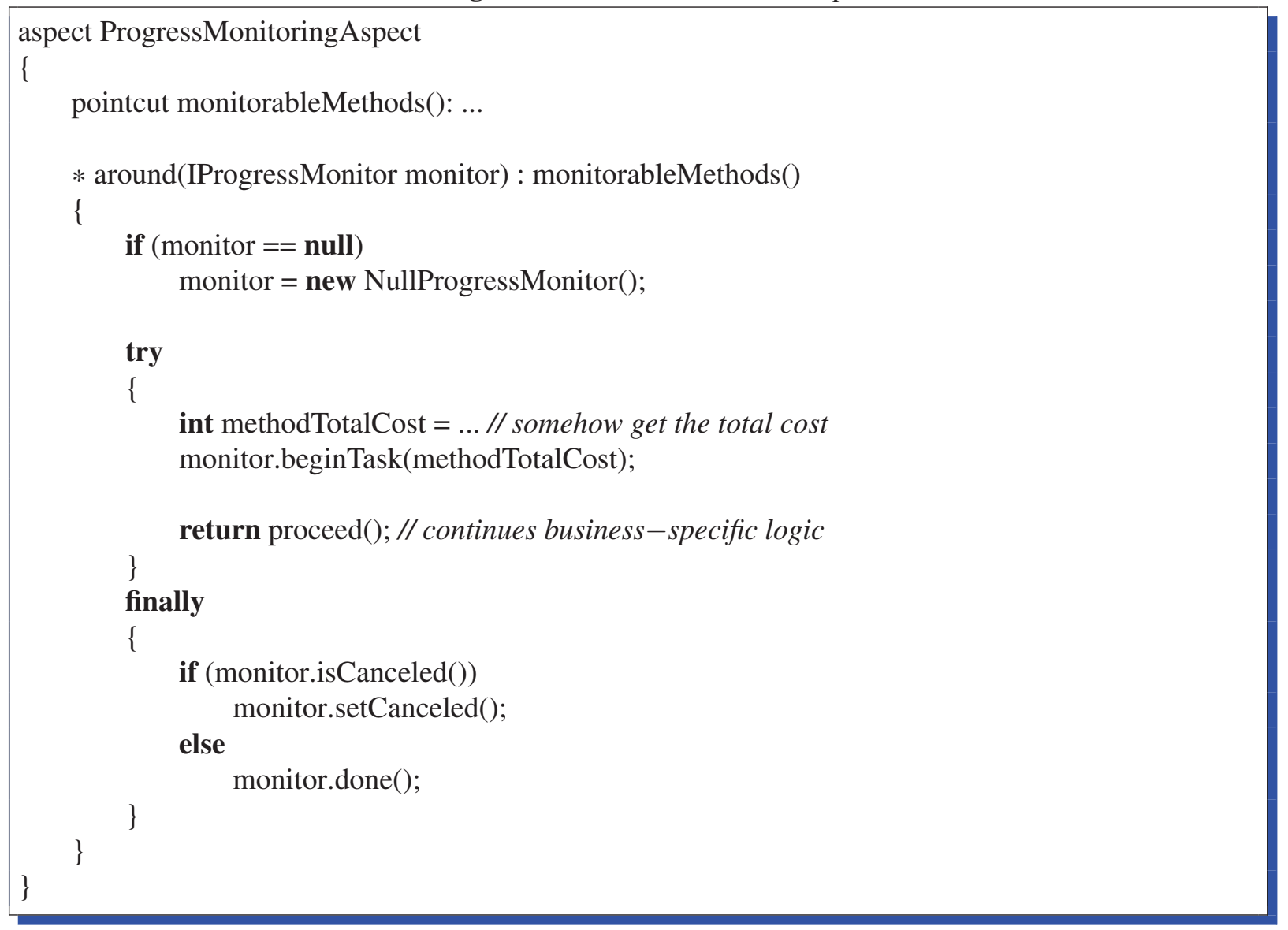

Thus, the example in Listing 2.6 would be replaced by that in Listing 2.9.

Listing 2.9 Example modified using Aspects

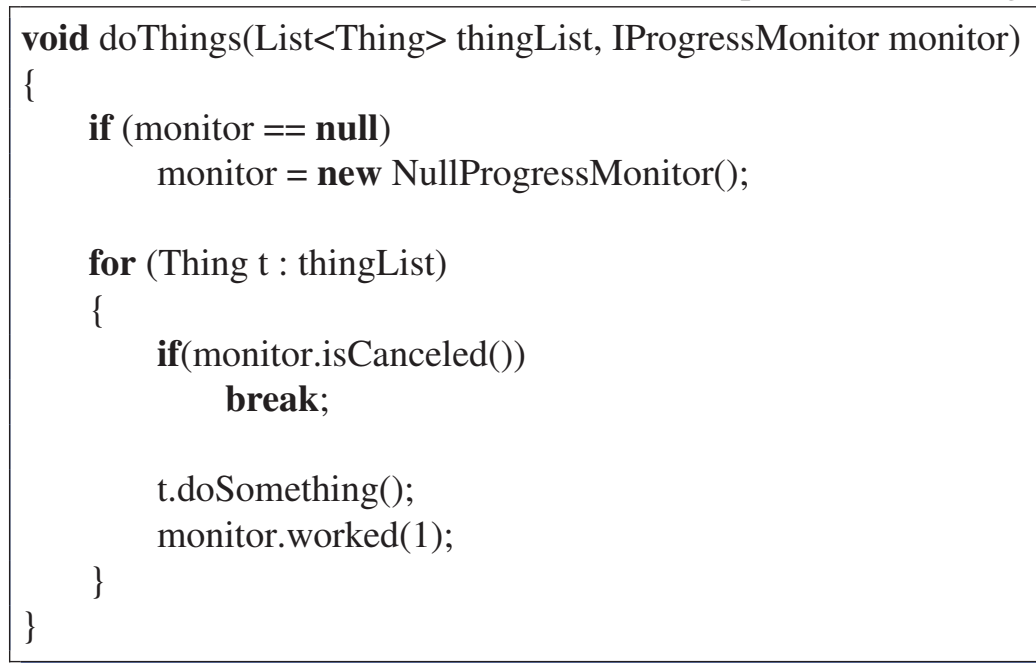

In the given examples, the monitor instance is shared by both the monitorable method and the advice implementation. The advice implementation focuses on the monitoring-related overhead that happens before and after the method executes. Using aspect-oriented programming may improve code modularity and replication, as the monitoring code is significantly separated from the main business code. We only consider two difficulties for the developer to deal with: 
- The aspect code is generic, as it would serve to all monitorable methods in the whole application. This means it cannot make any assumptions about the amount of work and the nature of the monitorable method. Setting the total amount of work of the monitor is a task intimately dependent on the method semantics and inputs. Therefore, we would need additional architectural elements for the aspect to gather abstract information from the concrete method, such as the total amount of work needed by the method. One can overcome this difficulty by defining an abstract aspect implementation concerned only with the main monitoring-related code, and then inheriting concrete aspects according to business-specific needs. Therefore, each concrete aspect implementation would properly handle the cost assignment. Alternatively, it is possible to use metadata-based conventions [GF10], such as annotations, and associate monitoring-related data to the monitorable methods, readily available for the generic aspect implementation to access - this approach is discussed in Section 2.4.

- Not all subtasks are methods. Aspects use pointcuts to determine points of additional code injection, but pointcuts define sets of methods to crosscut. The problem relies on establishing a one-to-one mapping between method calls and tasks. For example, one can create many subtasks within a single method implementation, such as in Listing 2.2. In this case, a single method call could generate a whole tree of subtasks. It is possible to overcome this difficulty for new service implementations, by simply introducing a coding convention that guarantees that every subtask is implemented as a new method invokation, forcing such a one-to-one mapping. For turning existing service implementations into monitorable services, this approach can require some refactoring, but it is still possible.

Despite the difficulties, one can certainly use an aspect-oriented approach to deal with the monitoring modularization problem in an effective manner, since it is possible to overcome most difficulties by introducing a few conventions and coding style. Therefore, we conclude that an aspect-oriented approach can improve an object-oriented approach, by simply moving part of the code from within the method to the scope of an aspect. Both the Eclipse approach and the solution proposed in this dissertation could be adapted to an aspect-oriented approach.

\subsection{The Metadata Approach}

Previous approaches can aid the implementation of monitorable services by providing classes, interfaces and aspects with built-in logic for dealing with execution state, progress and subtask management. One notable characteristic of the presented approaches is that they do not impose specific coding styles. This characteristic enables the developers to implement the business logic in their prefered way. In particular, they can freely decide the most appropriate way to organize their code, methods and classes. This is possible because all the monitoring-related logic are made explicit by adding code for invoking methods provided by the monitoring library. However, despite the benefits of freeing code style, the resulting monitoring-related code is often tangled with the business code.

The Metadata approach is based upon specifying coding conventions that allow the monitoring library (a class or aspect responsible for the monitoring concern) to dynamicaly infer the monitoring context at runtime, gathering context-specific information from metadata elements 
associated to the language static structures (such as classes and methods), by using reflection and annotation techniques. The ideia is based upon the principles of Convention over Configuration [Che06] and Attribute-oriented programming [GF10].

Most of the code tangling on the presented approaches derive from generating subtasks and updating the progress execution status. In order to eliminate this code tangling, one can establish a coding convention to guarantee that every atomic task (something that contributes to the overall progress execution) will be implemented as a separate method call. Therefore, by using this convention, it is possible to define a one-to-one mapping between the method calls and the monitoring subtasks. Furthermore, if we associate metadata to these methods, such as the operation descriptions and execution costs, we can dynamically gather information at runtime by using reflection, and use it to automatically report progress feedback. The Metadata Approach can be combined to any of the other approaches, including the solution proposed in this dissertation, in order to improve modularization, by introducing some conventions and programming style. By using metadata in conjunction with an aspect-oriented approach, the example presented in Listing 2.3 would be rewritten as in Listing 2.10.

Listing 2.10 Metadata example code

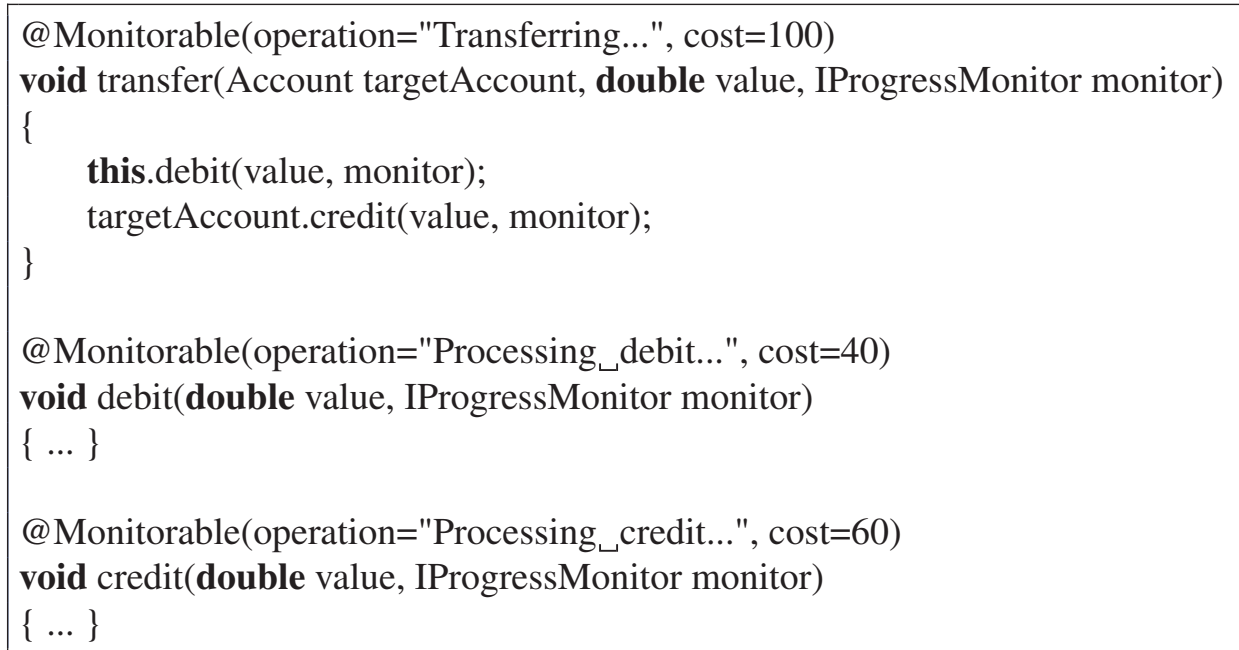

In order for the code in Listing 2.10 to work, we need to intercept every method invokation marked with the Monitorable annotation. This can performed by an aspect-oriented approach, code weaving or instrumentation technique. Once intercepted, at run-time, it is possible to reflectively gather the monitoring cost and operation description from the annotation attributes. Although the Metadata Approach can improve code modularity and readibility, we identify two major challenges to overcome:

- Costs can depend on the execution context. Annotations are designed for associating static data such as constant values. However, sometimes, the total cost of a method can depend upon the parameters and other contextual information which is only available at run-time, just as in the example of Listing 2.6, in which the total cost depends upon the size of a list. With an aspect-oriented approach, it is possible to deal with such a situation by using aspect inheritance, by implementing a specific aspect for the method. This would resolve the code tangling problem, but compromises regularity: some monitorable methods will only require an annotation, while others will require a custom aspect extension. Even if the costs can be assigned constant values regardless of the parameters, its 
cost can depend upon the context in which it is being invoked. For example, although the credit has been assigned a cost of 60 in the context of a transfer operation, it could be assigned a cost of 10 in the context of a reversal entry operation. Again, when combined with an aspect-oriented approach, it is possible to deal with this problem by extending a customized aspect and carefully specifying cflow for the pointcut, leaving the cost assignment as an aspect decision, but it adds complexity and compromises regularity.

- It is difficult to implement monitoring a posteriori. Since the Metadata Approach relies on coding convention, which forces developers to split up methods in a specific manner, whenever they need provide progress reports, it can be difficult to turn an existing nonmonitorable implementation into a monitorable one, possibly requiring too much manual refactoring.

Despite the challenges, one can use a Metadata Approach to improve a monitoring solution, making progress reports implicit, without interfering in the business code.

\subsection{The Workflow-based Design}

Virtually all software used in industry have the same "class of goal": to support business processes. Some processes are entirely automated, relying solely on communication among applications. Others also rely on people to initiate the process, approve documents the process uses, resolve any exceptional situations that arise, and more. In most cases, it is possible to specify a discrete series of steps, known as workflows, describing the activities, their sequence, conditions, people and software involved in the process. Once a workflow has been defined, a software application can be built, on top of its definition, to support the business process [Cha10].

In this context, workflow-based design have emerged in the past two decades, to support business processes modeling and implementation, as an interesting alternative to traditional architectures. Workflow aims at modeling and controlling the execution of application processes in dynamic and heterogeneous organizational and technical environments, without the need of a single line of code [Wes99]. One of the most interesting aspects of workflow-based design is the separation between business process code and the actual implementation — which by the way is often a requirement in Service-Oriented Architecture (SOA). Without workflow-based design, the typical scenario would be to write both the business logic and the actual implementation in traditional programming language, which results in no clear separation between business logic and its implementation.

Today, a large number of commercial applications and frameworks is available for supporting workflow-based development of business processes in applications [ECA08]. Activities are the building blocks of a workflow. An activity models an individual step in a workflow and these are linked together to form a flow. To illustrate the concept, Figure 2.3 demonstrates the workflow for our fictitious example in Listing 2.2.

Workflow-based designs are particularly interesting for providing monitoring capabilities. This is due to the fact that every workflow explicitly defines all activities and transitions of a business process, and the order in which they execute. If every activity of a workflow is monitorable, and has an associated cost in the context of the workflow execution, then the 


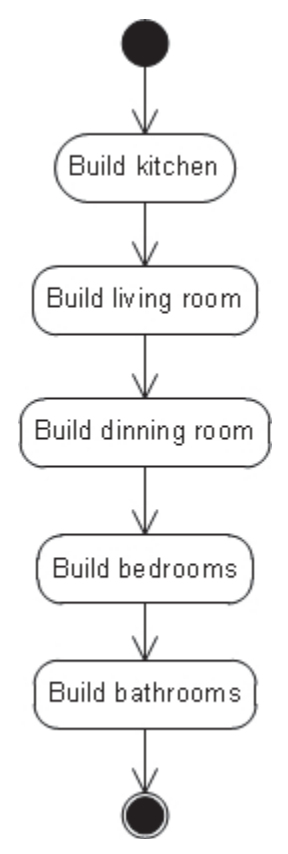

Figure 2.3 Workflow example.

workflow is also monitorable. For this reason, we can say that services designed as workflows are half-way of becoming monitorable.

A well defined workflow can be naturally translated into an object-oriented model that describes the main service algorithm flow. This characteristic simplifies the monitoring problem because it limits the possibilities and allows a regular and modular solution that can be generalized for making any workflow monitorable.

One could easily adapt each one of the twenty Workflow Patterns defined by Aalst in [WVDAB03] and make them monitorable workflow patterns. To illustrate, our fictitious example in Listing 2.3 can be easily converted to a monitorable workflow, as we demonstrate in Figure 2.4.

We can easily derive generalized rules for transforming non-monitorable workflows into monitorable ones. Figures 2.5, 2.6 and 2.7 show these generalized transformation rules.

Before actually running its first activity, a monitorable workflow must calculate total relative work units for the workflow overall execution in order to provide progress feedback to the higher-level layer. In an object-oriented representation of the workflow, this task can be very easily automated, due to the fact that we would have classes for representing each node in the workflow, along with their connections, so that we could dynamically traverse each node of the workflow and sum up their associated costs relative to the workflow.

Between each activity-activity transition, we must report updated workflow progress state to the higher-level layer and verify cancelation request state. If cancelation has been requested, we must rollback all already performed activities, report cancelation acknowledgment to the higher-level layer and finish workflow execution. Again, in an object-oriented representation of the workflow, this behavior can be easily automated, as long as the activities provide an abstract rollback functionality that can be invoked after the activity has already completed, and the workflow execution engine provides tracking of completed activities.

Before finishing the workflow with a transition to final state, we must report progress com- 


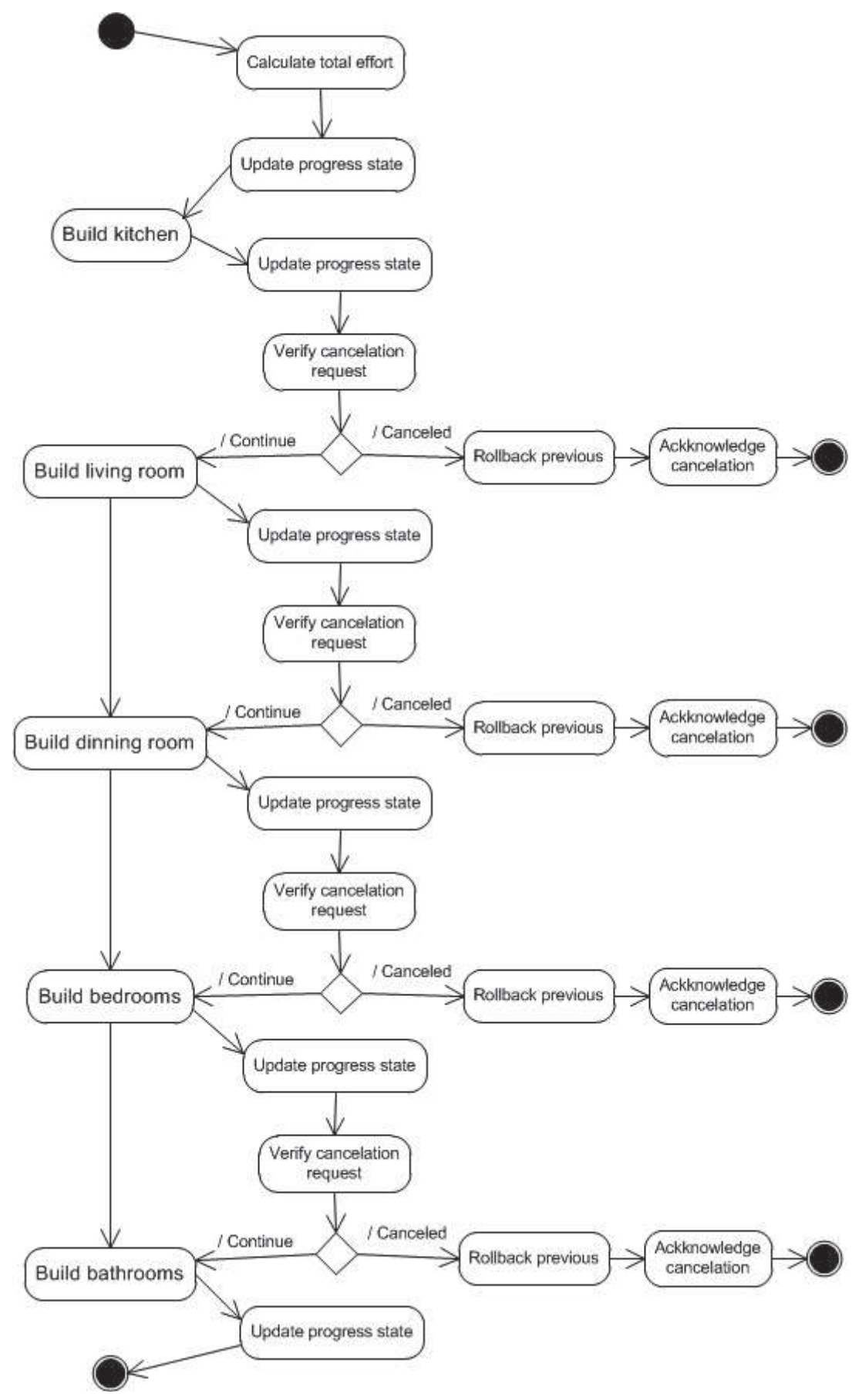

Figure 2.4 Monitorable workflow example.

pletion state to the higher-level layer. Again, this task can be easily automated in an objectoriented workflow representation.

One might notice that an activity can be an atomic and simple activity, or a complex activity that encapsulates another whole workflow inside it. In such case, if the chained workflow encapsulated by an activity is also monitorable, then its partial progress update reports would be scaled in relation to the higher-level workflow context. This scaling and monitoring of chained workflows can also be completely automated by the workflow execution base engine. 


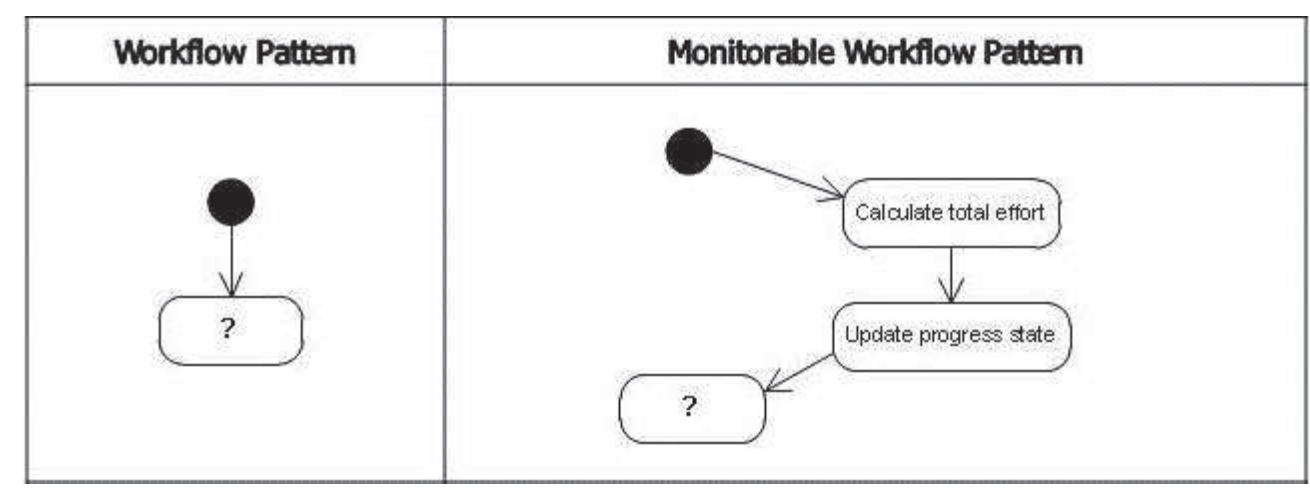

Figure 2.5 Transformation rule for workflow initial state transition.

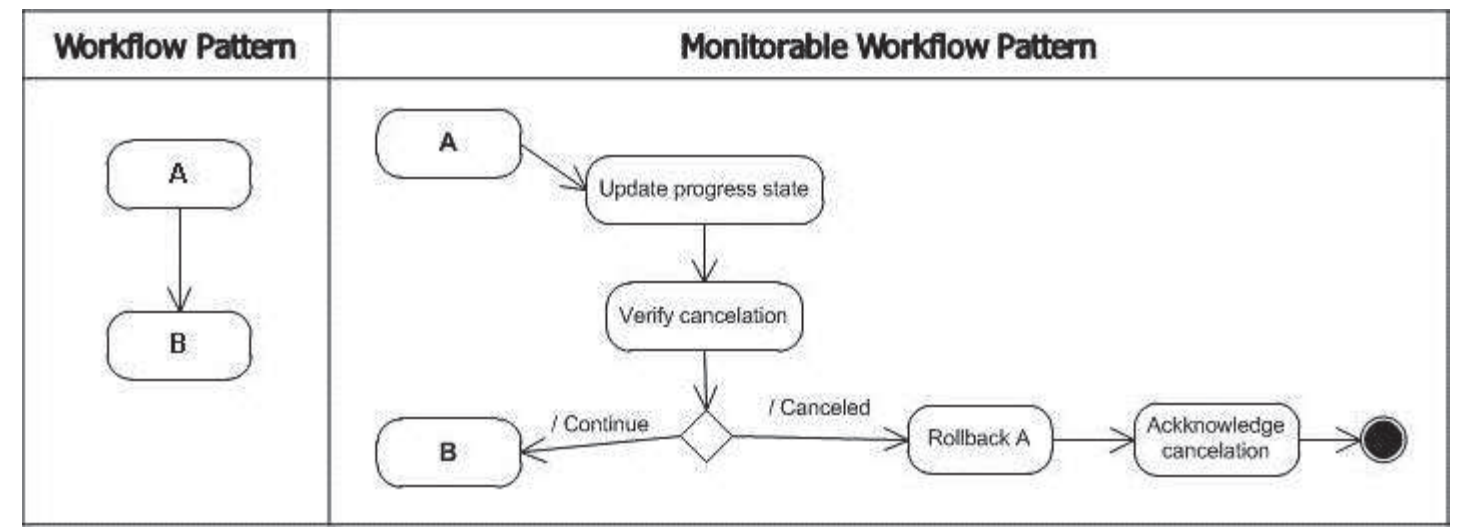

Figure 2.6 Transformation rule for activity-to-activity sequential transition.

\begin{tabular}{|l|l|}
\hline Workfiow Pattern & Monitorable Workflow Pattern \\
\hline
\end{tabular}

Figure 2.7 Transformation rule for workflow final state transition.

With a generalized solution to make any workflow monitorable, one could extend an existing workflow framework in order to add monitoring capabilities. For example, we could define abstract classes MonitorableActivity and MonitorableWorkflow and put all the monitoring logic inside these abstract class implementations. Then, a business process developer would simply extend those classes, provide proper costs for the activities and would be done.

In the context of our work, although we encourage using workflows for modeling and implementing business processes of application services, because of its potential for separating business logic from the implementation details and stimulating reuse for business algorithms [WVDAB03], still we prefer not to assume any particular type of design and implementation. 
The reason for this is simple: not all services are implemented by means of workflow design. We intend to provide a framework that would be useful for both workflow-based and by traditional implementations that mix processes within the programming language algorithm. Furthermore, we illustrate how the framework can be useful for both situations.

Also, workflow-based services are often implemented by using an existing framework for representing workflows and activities. Even if we decided to deal, in this work, solely with workflow-based service implementations, we would have to choose a specific workflow framework to extend, due to the fact that each workflow framework has its own peculiarities that would have to be considered for us to add monitoring capabilities to the framework. In any case, the general purpose monitoring framework specified in this work would be of great utility for one interested in extending a workflow framework for adding monitoring capabilities.

Our work presents a general purpose framework along with extended libraries that do not assume any workflow-based architecture, but could be equally useful for working in conjunction with workflow frameworks, such as the Windows Workflow Foundation [Cha10] — Microsoft extensible workflow framework, built on top of .NET Framework 3.5, for implementing business processes. Such workflow frameworks can be extended, reusing the proposed solution, to add monitoring capabilities to any workflows.

Despite the modularity and automation benefits of using strictly workflow-based design for implementing services, still we identify one major inherent drawback of its usage: activities might not be easily decouplable, since they can share too much context information among them, and hardly depend on the other activity. In typical non-workflow-based implementations, these activities would be tangled to each other and it is often difficult to distinguish which code belongs to each activity, with the advantage of simplifying implementation, model and context sharing between activities. Workflow-based implementation tends to model autonomous activities - just as if activities were independent agents that contribute to the workflow execution and obliges modular implementation of activities. This means that these complex dependencies and shared context between workflow activities must also be modeled, which may lead, in these cases, to a great increase of model and implementation complexity. In summary, the more independent and autonomous the activities, the easier it is using workflow-based design for business process implementation.

\subsection{Software Performance Engineering}

Interest in measuring software performance dates back from the development of the very first computer systems [Jr71]. Many works and techniques have been developed towards evaluating program performance. An entire discipline called Performance Testing has emerged for determining and reporting current performance of a software application under various parameters [Bar04]. In this context, Software Performance Engineering is the process by which software is tested and tuned with the intent of realizing its required performance.

Software Performance Engineering techniques are used by verification teams to ensure that the system under test meets its performance specifications, by performance modelers to build and validate models, and by designers and developers to find and fix hot-spots in the code [MW07].

Software monitoring techniques can be broken down into two broad categories: instrumentation and profiling. Instrumentation is the insertion of probes into a system to measure some 
sort of events [MW07]. There are several different techniques to add instrumentation to programs. It can be manually performed by the developer by explicitly adding instructions along the code; by using tools that automatically changes source code; by using compiler-specific options; by using binary translation techniques; at runtime when using virtual machines, etc [Wik10]. Profiling is a technique for generating histograms of the execution of a program. It can be generated by using instrumentation, by statistical sampling or by running the program on a virtual machine [MW07]. It consists on counting the execution of instructions, methods and operations in the program and measuring execution times.

One of the main purposes of currently developed techniques is to improve software quality by identifying performance bottlenecks and test program performance with different scenarios and inputs. These techniques are very well established at the verification level. However, they are less well established at earlier stages in the life-cycle of a product [MW07]. In [ADM01], Malony and Helm have identified some obstacles in particular to the adoption of these techniques:

- A lack of theoretical justification for the known approaches for improving performance. Tools will provide measurement data, but expert interpretation is still required to fix problems. The lack of formal explanations makes it difficult to actually automate the process of fixing the problems.

- A conflict between automation and adaptability in that systems which are highly automated but are difficult to change, and vice versa. As a result, no tool does the job the user needs, so the user goes and invents one. Furthermore, various tools have different forms of output which makes interoperability challenging at best.

- In distributed systems, events from different systems need to be correlated. Today's systems are often composed by sub-systems from different vendors. Establishing local causality across a distributed system requires great effort.

Besides software optimization and post-release performance evaluation, in the context of this work, we consider adding a new obstacle to the list:

- Lack of techniques that automatically deal with performance monitoring as part of the software functional requirement, providing rich usability and feedback to the user while executing the tasks. Here, the requirement is not a matter of executing faster or slower, but to provide the end-user with progress execution feedback so that he can monitor what is being executed and the percentage done while the requested application services run.

Although it is not the purpose of the profiling technique to provide program execution feedback to the end-user in a production environment, it still relates to this work as it is an execution monitoring technique to be used by software engineers in testing environments.

Program profiling is very useful for observing the execution of a service and to present quantified measures of what has already happened, but it often does not help much on predicting measures of next operations, with different contexts and input parameters. Thus, program profiling does not provide the overall percentage of progress completion while the service is still executing. 
Current approaches for providing progress execution feedback to the end-user, such as the one briefly discussed in the previous section - a framework-based approach developed by the Eclipse Community - requires the software engineer to estimate the cost of each suboperation that composes a major operation. Once familiar with profiling techniques, one can become tempted to use them for estimating the cost of the operations in a service - or even dream of automating the operation costs assignment - , but it will certainly need to overcome two major challenges:

- High-level operation costs are not uniform. In real environments, operation costs are inherently unpredictable, due to specific operating systems processes/threads prioritization policies, I/O-dependent operations, memory cache state, etc. This means that even running a service with the same inputs, a program profiling can measure different execution times when called twice. For example, on the very first execution, every data required by the service would be stored in the hard disk, while at the next execution the data would be readily available on the memory; In addition, a different system process could simultaneously start execution, sharing the system resources and thus interfering on the execution times.

- Costs of operations are input-dependent. The costs of the suboperations that compose a major operation can unpredictably change according to the inputs. This means that what can be pointed out as a high-cost operation in a given execution scenario could also be pointed out as a low-cost operation in a different one. Weights of suboperations depend on the input. By using a program profiling, one can discover the weights in a given inputspecific scenario but does not find the function that calculates the weights for any given scenario. This function is needed for an accurate monitoring and often requires human analysis. For example, in a billing system, calculating the final value of a fixed-price contract can require less processing than a corresponding variable-price contract, such as a collar.

One can consider overcoming these challenges by using certain types of auto-adjustable neural networks (or other statistics-based technique) to dynamically recalibrate the costs at run-time and gradually converge them to more accurate operation costs as long as new service executions are performed. However, in practice, such a solution would face two major difficulties:

- Business processes can have discontinuities. Just because doubling a given input variable in the last service execution resulted in doubling the execution time of a certain operation and reduced another operation time to a half, does not mean that the same pattern can be interpolated when other arbitrary value is assigned to the same input variable. Complex business workflows can be full of breaking conditions, thresholds, and many alternative execution paths which may depend on hundreds of input variables. Such situation would break behavioral linearity, making it very hard (possibly impracticable) for learning algorithms to converge to accurate weights.

- Training algorithms can be costly. Even if there is a certain linearity in the service execution and only a few input variables, training algorithms often require large amount of sampling in order for it to accurately converge. Therefore, it can take some time for it to provide effective results. Also, daily maintenance in different software components 
could affect the performance of workflows, requiring a new training for every change in service depending components.

Despite the challenges regarding the use of profiling techniques to automate costs assignment, it is definitely possible to use these techniques in a verification phase in order to validate a given set of possible costs against the actual execution time for a given input scenario. In such case, profiling techniques can be useful for assisting on manual tuning of costs. Operation cost estimation is inherent to the monitoring problem and we currently suspect that there is no promising solution for automating this process in a simultaneously generic, unfailing, accurate and practical manner. Further, we discuss a possible approach to make the process of defining operation costs more systematic.

\subsection{Chapter Summary}

This chapter presented some existing approaches and possibilities to support monitorable services implementation. It detailed different strategies for implementing monitoring in different approaches, showed examples, and discussed their advantages and disadvantages. These results serve as basis for the specification and development of our proposed solution, in an attempt to improve advantages and avoid disadvantages of these approaches. 


\section{CHAPTER 3 \\ Monitoring Framework}

This chapter presents a general purpose framework, specifically designed to aid the development of monitorable services. Monitorable software systems can be developed on top of this general purpose framework.

The framework provides the concepts in the domain of execution progress monitoring by defining classes and interfaces that represent generalized abstractions and should be reused and extended by applications that need to address the monitoring requirements. The framework aims at serving as basis for general applications. Therefore, its usage does not impose specific algorithms, heuristics or design assumptions. Different applications in different contexts may want to add additional features and change the default behavior regarding execution monitoring.

The framework development represents the core contribution of this dissertation. It is the basis for the development of the libraries that are presented in Chapter 4, and it defines the fundamental model for representing the monitoring entities, the contracts and communication protocol that rules consumer-provider interactions.

First, we introduce the main concepts in the domain of execution progress monitoring. Then, it is defined the main requirements and use-cases of the framework. Finally, it is presented the class diagrams and interfaces that represent the main entities and abstractions, showing how they relate to each other and explaining the role they play in the application context.

\subsection{Concepts and Requirements}

In order to introduce the concepts related to the execution monitoring problem, it is important to understand an application as a set of interactions between Consumers and Providers. Most software system implementations consist of entities consuming services provided by other entities or by themselves. We are interested in monitoring the execution (or consumption) of these services at run-time, on-line, as consumers and providers interact. Consumers and Providers are fundamental concepts for understanding the monitoring framework.

The object-oriented paradigm naturally stimulates the design of programs by conceiving entities (classes) that conform to contracts and provide services (methods) for consumers (other classes) [Som07]. With modularity, the implementation of a service usually involves many interactions between consumers and providers, leading to the chained execution of smaller services. Services are usually built by a composition process. The notion of a service consisting of a combination of other smaller services must be considered in the monitoring model, since the strict monitoring of a major service implies monitoring every other cascading service invoked in the execution chain. Put simply, if a major service is strictly monitorable (not partially), then every underlying service that compose the major service must also be strictly monitorable.

The framework proposed in this work aims to aid software developers with the implemen- 
tation of monitorable services, attempting to improve productivity, maintainability and overall code quality. The developer responsible for the implementation of a specific service might want to invoke other services implemented by someone else. This is a very common scenario even for small development teams. Therefore, the framework model must allow the developer not to know the implementation details of services implemented by others. Put simply, if a developer needs to invoke a service implemented elsewhere, it should be able to abstract the monitoring implementation details.

According to [Bec07], there are three styles of use that a framework can support: instantiation, implementation and configuration. The framework proposed in this work is mainly based on an implementation style of use, by only defining interfaces and abstract classes, leaving the client to implement the framework interfaces and extend the framework classes to include logic during its execution. Since the framework is intended for general purpose usage, we avoid concrete implementations and focus on the basic protocol for consumers and providers to communicate.

Non-trivial services often have their execution logically divided into Operations (steps) which could be individually monitored by the Consumer. Operations are a set of tasks or subtasks performed by a service during execution. Operations are semantically complete and independent from the major service semantics. Their meaning are often understandable out of the major service context. For example, an application service that performs updates to a database after gathering information from the internet can be logically divided into some $O p$ erations whose execution could be monitored by the end-user: establishing connection to the internet; performing authentication; downloading web pages; processing information; establishing database connection; creating database transaction; executing sql queries; etc. Operations have their own inputs and outputs; they start and finish execution and work as a monitorable service inside another service. By definition, every monitorable service, even the most trivial one, must perform at least one operation which represents the overall service execution.

It is important to notice that there is no direct relation between the notion of Operation and the modularization strategies adopted by developers in the service implementation. More precisely, typically there will not exist an one-to-one mapping between logical Operations (which represent the end-user high-level point of view) and the physical methods implemented as part of a static architectural and design strategy. Thus, an Operation can be defined as a set of instructions in the implementation of service, which might involve calls to many other services in different entities or simply aggregating language constructs and statements (loops, conditionals, declarations, etc.).

The framework defines classes for representing the concept of Operation and enables their management and control by the application.

Every Operation takes some time to execute. Thus, in the context of a service implementation, each Operation will have an associated execution cost. At run-time, Operation state changes must be tracked and managed concerning at least two aspects:

- Status Control. Enable tracking the current state for an Operation (stopped, executing, cancelation requested, canceling, canceled, interrupted or finished).

- Progress Control. Enable tracking the percentage of execution remaining for the Operation to be finished. 
Services usually output information to the consumer. The main purpose for a service execution is often to process some sort of information to the consumer (possibly the end-user or a sub-system). However, while a service is executing, the service provider may already have useful information about the process to send to the consumer. Errors, alerts and untypical situations are examples of information that could be shown to the Service Consumer while the execution is still in progress.

In this context, the framework must provide a built-in mechanism for sending asynchronous Notifications to the Service Consumer (messages, errors or alerts) while the service is still executing. Therefore, the framework must provide sending and receipt features, for both service provider and consumers.

Notifications are generated as a result of Operations execution. Since the Operations process in chain, the notifications can be considered in the same manner, based on the concept of parent notifications and child notifications, which can be represented as a tree. Figure 3.1 shows an example of an operation tree. The framework defines classes for representing the Notification concept and allows its management and control by the application.

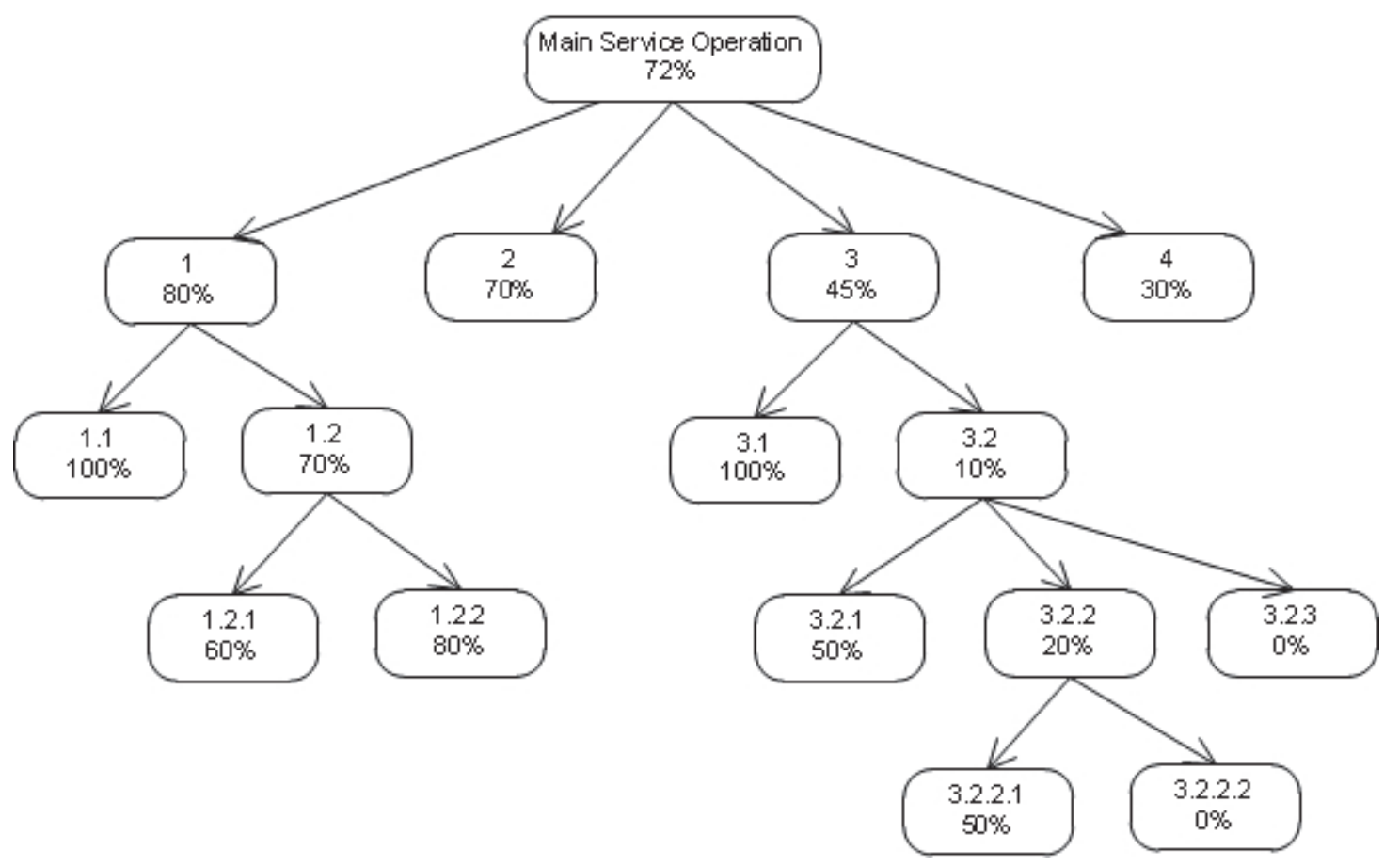

Figure 3.1 Example of an operation tree

In the Figure 3.1, the main operation splits into four independent operations, each one with its own progress state. Some of these operations also split into other operations, which are also independent and have their own progress state. One could notice that an operation progress percentage is not necessarily equal to the average of the percentage of its immediate child operations percentages. This is due to the fact that child operations might have different lengths and weights in the context of the main operation. Due to the general purpose characteristic of the framework, each operation must define the most appropriate aggregation logic for updating its own progress state. (An application extending the framework for usage in a more specific scenario would consider more appropriate to establish a fixed aggregation strategy. This subject 
is covered in the next chapter, when we present concrete libraries that extend the framework).

Although we focus on monitoring the progress execution, the general purpose nature of the framework should allow an extended solution to incorporate additional monitoring capabilities, such as throughput, traffic rate, memory usage, or other business-specific monitorable dimension. The principles for the service provider to report richer monitoring information remain the same.

In addition, besides representing Operations and Notifications, in spite of not being the focus of this work because it is not directly related to the monitoring problem, the framework also provides support for the cancelation of services that are still executing. We added this functionality because the structures provided by the monitoring concern are also well suited for implementing cancelation control. Therefore, it provides a default protocol that enables the service consumer to request cancelation of a service and enables the service provider to respond to the consumer request. The service provider must be prepared to receive and properly handle these requests considering its current state.

The framework must be constructed in such a way that it does not impose - or at least minimizes - design restrictions to the application architecture. Considering an application completely developed without execution monitoring capabilities, it should be easy to adjust and adapt its implementation by using the framework to support it. For this requirement to hold, a monitorable system architecture should not be distinguishable from a non-monitorable system architecture. By "system architecture", we mean the system's layers, boundaries, facade interfaces and core component associated contracts.

Once the framework has been briefly characterized with respect to its core concepts service consumers, providers, operations and notifications - , it is possible to enumerate its functional requirements:

RF01 Allow tracking current progress state of services execution.

RF02 Allow sending/receiving notifications during the service execution.

RF03 Provide built-in mechanism to support services cancelation.

RF04 Provide control over finished and running operations.

RF05 Provide support for monitoring chained operation execution.

RF06 Provide support for monitoring concurrent operation execution.

RF07 Provide support for monitoring distributed services execution.

RF08 Enable multiple monitoring entities for a single service provider.

In addition, it is important to consider some non-functional requirements:

RNF01 The implementation of monitoring support must not interfere on the application functional behavior.

RNF02 Notifications and progress feedback must be sent asynchronously, without interfering on the service execution flow. 
RNF03 It must be easy to integrate to existing application designs and architectures and must not meaningfully increase code complexity.

RNF04 The implementation of monitoring support must not degrade the application execution performance.

\subsubsection{Framework Use-Cases}

The framework must provide functionalities to satisfy both consumer and provider requirements:

- For the providers' perspective. It must provide a set of components for the developer to implement monitorable services.

- For the consumers' perspective. It must provide a set of components for the information output by the provider to be received and monitored by the consumer.

Figures 3.2 and 3.3 show Use-Case diagrams for both the Consumer and the Provider.

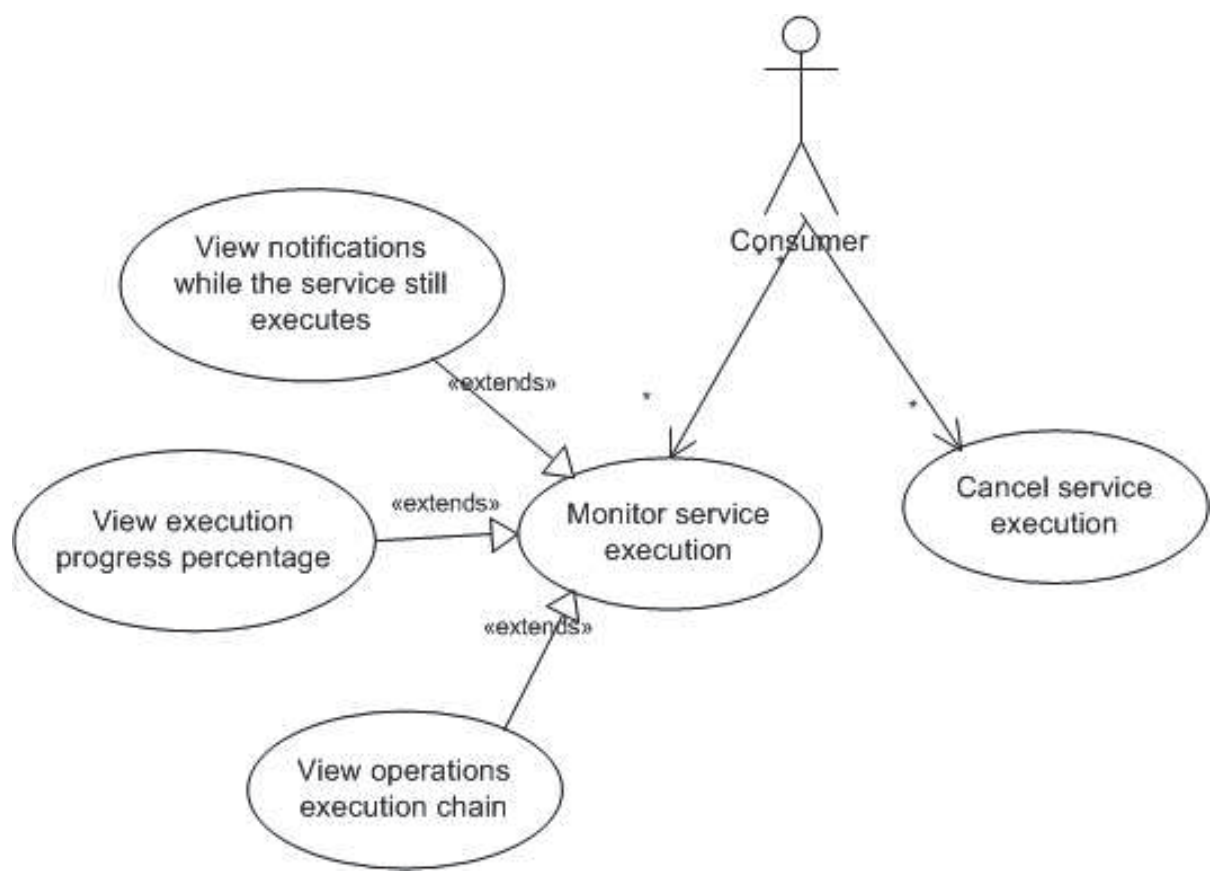

Figure 3.2 Consumer Use-Cases

Both consumers and providers share some core concepts, such as operations and their possible states, and thus the framework must provide classes and interface definitions that represent all fundamental concepts inherent to the monitoring domain. In addition, the framework must establish a set of interfaces along with an usage protocol. These interfaces must be implemented by the monitorable services providers in order to allow the consumer to access monitoring information. The next section describes the packages and each entity that composes the framework. 


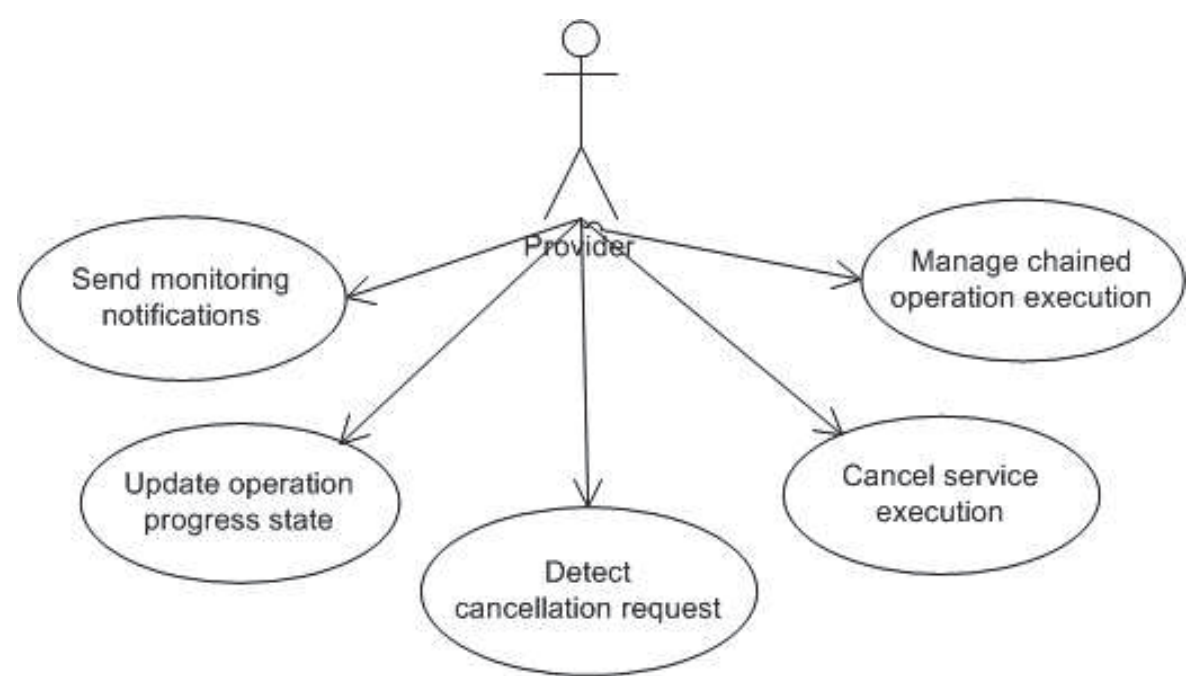

Figure 3.3 Provider Use-Cases

\subsection{Framework Architecture}

The framework is organized in three packages: Operations, Notifications and Monitoring. Figure 3.4 shows the relationship diagram.

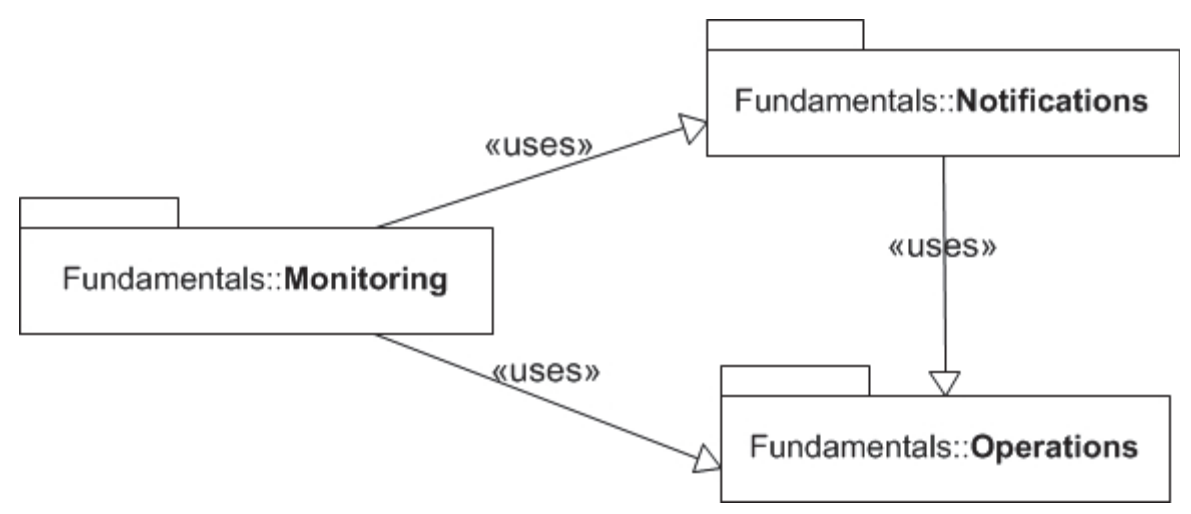

Figure 3.4 Framework package diagram.

The package organization criteria is based on the three domain concepts involved. There are three packages for representing the three main concepts in the framework domain: Operations, Notifications and Monitoring.

Before presenting the class diagrams, it is important to become familiar with the Observer Pattern ([GE95]), as it plays a fundamental role in the class definitions, for supporting message exchanging and state change notification. The next section provides a brief description of the Observer Pattern and explains its importance in the framework context.

\subsubsection{Observer pattern}

The intent of the Observer Pattern is to "define a one-to-many dependency between objects so that when one object changes state, all its dependents are notified and updated automatically" 
[GE95].

The Observer Pattern allows for the decoupling of objects that need to notify an event and objects that need to receive the notification. In other words, it decouples notify-ers and notifyees [AS00].

Whenever there is a set of objects that need to be notified whenever an event occurs, and the notification needs to occur automatically, the objects sending and receiving the notifications must not know each other (decoupling), the Observer Pattern will often be a suitable solution. In our framework, operations will notify consumers (or any other entity interested in receiving the notifications) every time the state changes or the overall percentage of service execution changes.

The entities that are registered to be notified are called observers and the entity that triggers the event is called subject.

The subject must implement methods (attach and detach) that allows the observers to register themselves to receive the notifications.

The observers must implement a method (notify) that allows the subject to call for notifying registered observers every time the event occurs.

Figure 3.5 illustrates the pattern interfaces.

\begin{tabular}{|l|l|}
\hline \multicolumn{1}{c|}{$\begin{array}{c}\text { «interface» } \\
\text { ISubject }\end{array}$} & $\begin{array}{l}\text { 《interface» } \\
\text { IObserver }\end{array}$ \\
\hline $\begin{array}{l}\text { +attach(in observer: IObserver) } \\
\text { +detach(in observer : IObserver) }\end{array}$ & \\
\hline
\end{tabular}

Figure 3.5 The Observer pattern.

This dissertation describes the framework by using only classic and universal object-oriented structures which are easily representable by UML standard notation [Rat99a]. In this sense, the Observer Pattern is appropriate because it conforms to classic object-oriented structures and UML notation. From another point of view, it may not be the most practical solution in specific programming languages, such as Microsoft $\mathrm{C \#}$, which defines native language constructs (events and delegates) [Lib08], non-standard and non UML-representable, that simplify sending and receiving notifications seamlessly, and without loosing decoupling capabilities, being an adequate substitute for the Observer Pattern. However, for the purpose of this work, it is more appropriate to use the Observer Pattern, because we want to propose a platform-independent solution to the monitoring problem.

\subsubsection{Notifications Package}

The Notifications Package provides classes and interfaces for representing the Notification entity. Figure 3.6 presents all related entities in a single class diagram.

The main entities in this package are: Notification abstract class, INotificationsProvider interface and the three notification types that inherits from the Notification class: MessageNotification, WarningNotification and OperationNotification. The next sections describe each one of them in details. 


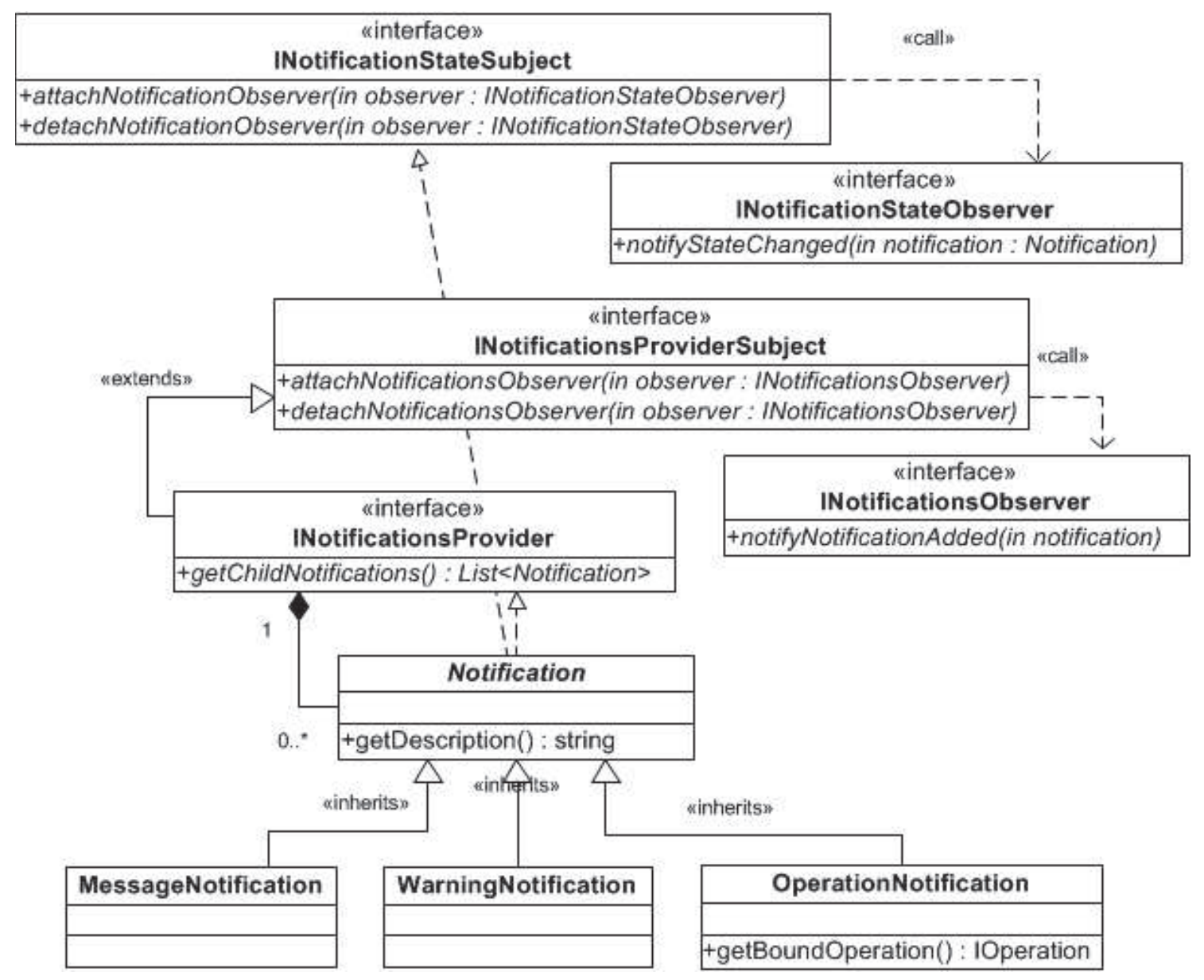

Figure 3.6 Notifications class diagram.

\subsubsection{INotificationsProvider Interface}

The INotificationsProvider interface represents an abstract source of notifications. Any entity that generates notifications should implement this interface. Operations are a clear source of notifications, since they are allowed to have child notifications. By using this interface, Consumers can access the set of notifications generated since the main operation started execution. The method getChildNotifications allows retrieving a list of notifications directly generated by the INotificationsProvider.

The INotificationsProvider interface implements the Observer Pattern by extending the INotificationsProviderSubject interface. Entities interested in receiving notifications can implement the INotificationsObserver interface in order to attach themselves as observers, so that they are notified whenever a new notification is generated.

\subsubsection{Notification Abstract Class}

The abstract class Notification represents a generic notification generated by a service within its execution context. The specific types of notification, with specific characteristics and behavior, must be extended from the abstract class. Thus, the abstract class provides common behavior that is inherent to any kind of notification. 
Service notifications can be represented in a tree, just as operations, as demonstrated in Figure 3.1. Figure 3.7 shows an example of a user-interface component for displaying notifications as a tree to end-users. The framework supports the notification tree by allowing notifications to generate child notifications. This is done by implementing the INotificationsProvider interface. This implementation is clearly a variation of the Composite Design Pattern presented in [GE95], but with a higher level of decoupling, as the Notification class does not directly host the list of child elements; it does it indirectly by implementing the INotificationsProvider interface.

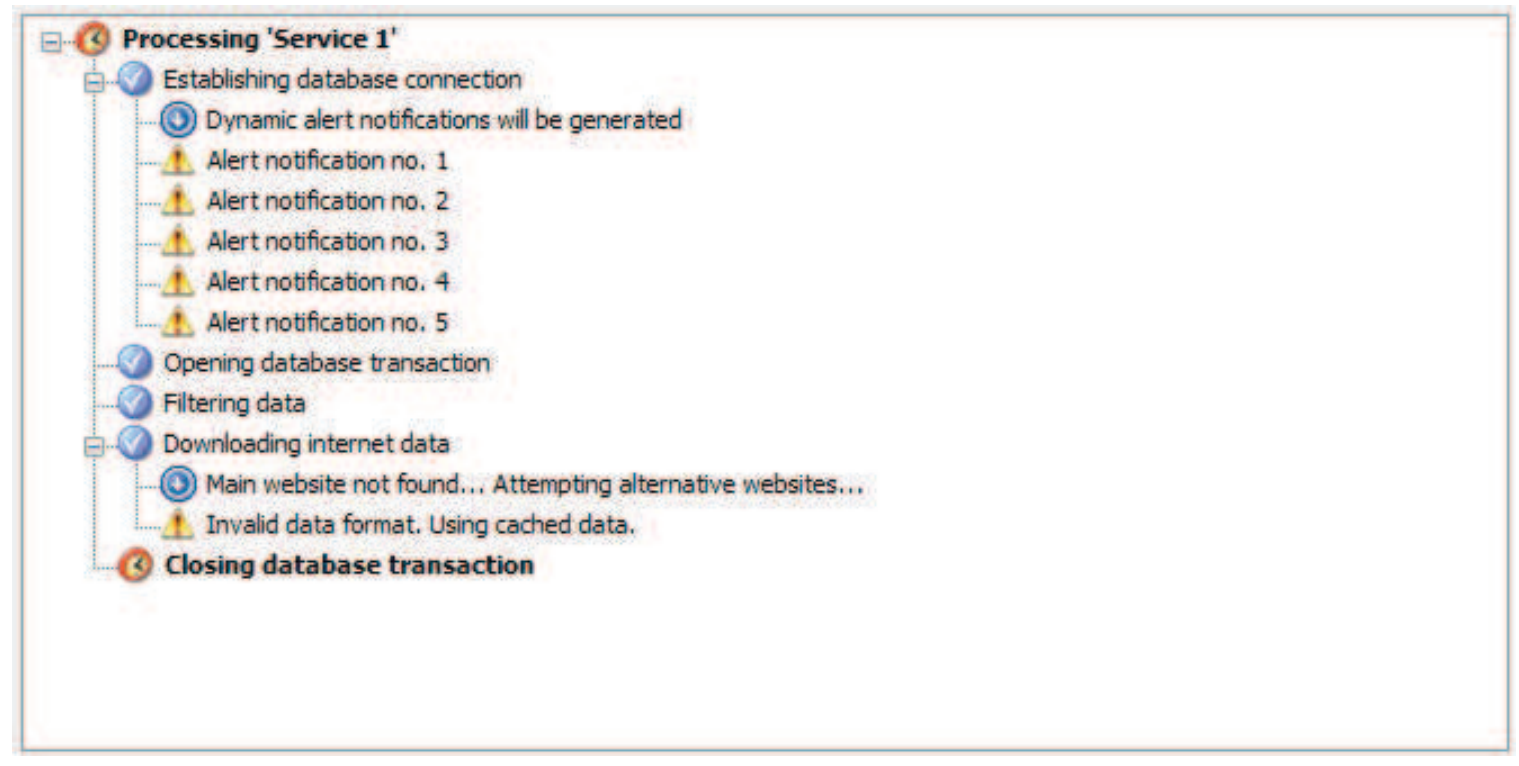

Figure 3.7 Example of notification tree in a user interface.

In addition to the INotificationsProvider interface, it also implements the INotificationStateSubject interface, which allows notifying when the notification state changes. For example, it is useful for dynamic notifications that changes its own type, description or any associated data while the service executes.

One could inquire whether the model could have been simplified by merging all interfaces into a single one, since the Notification abstract class implements them all. However, it was an intentional project decision to split them up, because each one of them represents a different and independent responsibility. For example, it is possible for an entity to be a source of notifications, while not being a notification itself. This is the case for the IOperation interface described in the next section. From the observer perspective, this separation can also be convenient, since it is possible for a given entity to be interested in receving only one particular notification event and not the other. In such case, it would suffice to implement only one of the Observer interfaces.

Besides the members added in order to implement the interfaces, the abstract class Notification contains only one additional method named getDescription which provides a textual description for the notification in natural language.

The framework supports three native built-in types of notification: (1) simple message, (2) simple warning and (3) operation-bound notifications. Each one of them has its own class definition inheriting from the Notification abstract class.

Specific applications could extend the framework by inheriting from the abstract class and 
adding different types of notifications with specific behavior. The message and warning notification types are static and very simple. They do not add any specific behavior to the Notification class other than implementing the getDescription method.

On the other hand, the operation-bound notification adds some specific behavior. Every operation has at least one associated notification. The notification is generated by default and has the purpose of describing the action currently performed by the operation. This special notification updates itself automatically as the operation changes state. Thus, the operationbound notification acts as observers of changes in their associated operation current state.

\subsubsection{Operations Package}

The Operations Package provides classes for representing the Operation concept. Figure 3.8 presents all related entities in a single class diagram.

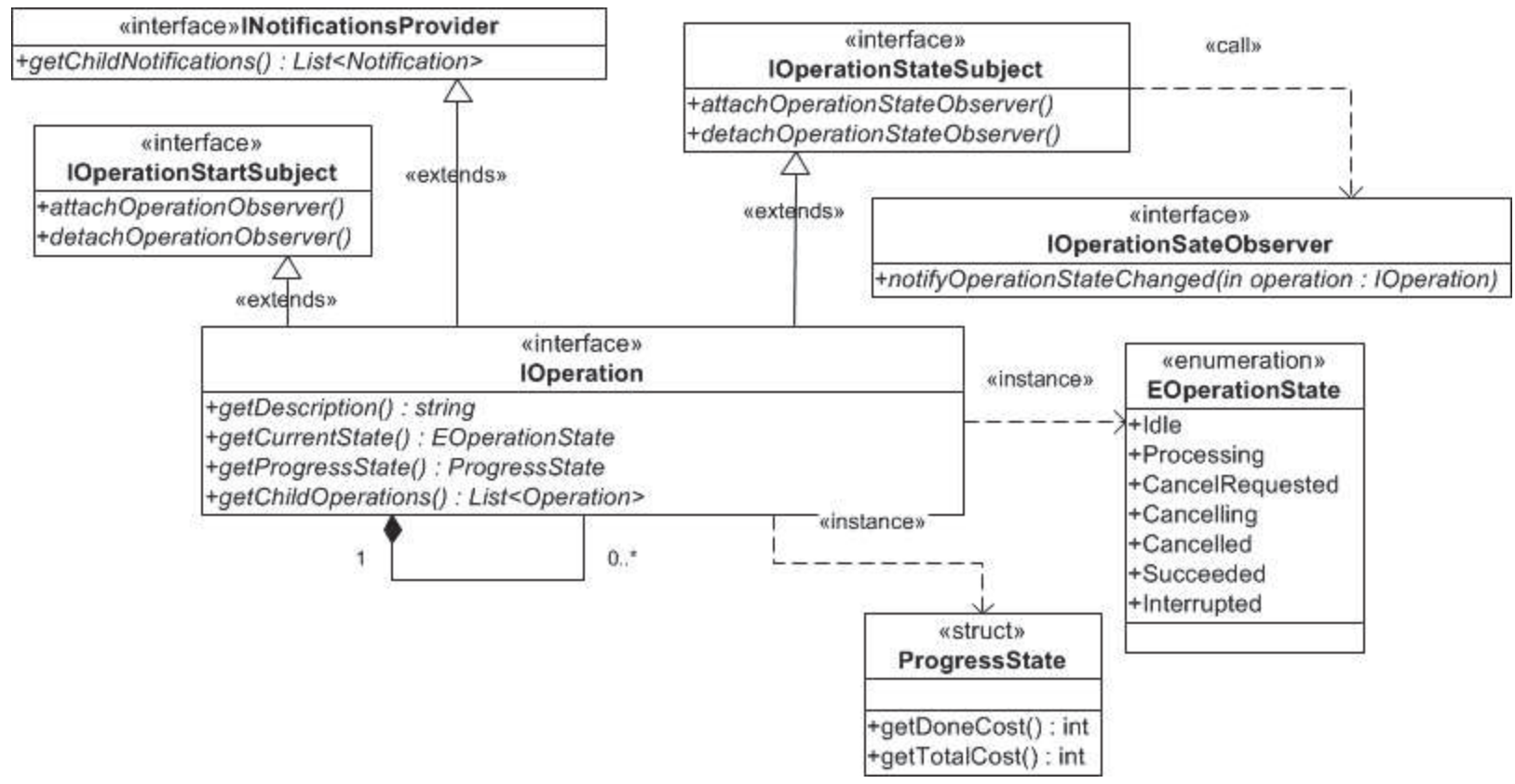

Figure 3.8 Operations class diagram.

As explained in Section 2, for this framework purpose, any service can be logically interpreted as the execution of a set of operations. In order to support the services representation in the framework model, the Operations Package provides three main entities: IOperation interface, EOperationState enumeration and ProgressStatus structure. The next sections describe each entity individually.

\subsubsection{IOperation Interface}

The IOperation interface is the foundation for the service execution monitoring framework. Every monitorable service must have exactly one operation (the main operation) representing the overall execution progress of a service. Every other operation will be a child (or descendant) of the main operation. Given an IOperation, it is possible to monitor its state changes and track execution progress percentage. 
Every operation is a source of notifications, for it can notify the higher-level tier with messages, warnings and providing operation progress feedback. Thus, the IOperation interface extends INotificationsProvider interface (see Section 3.2.2.1).

An operation can also have suboperations. The IOperation interface extends the INotificationsProvider interface, which allows the consumer to receive notifications when new suboperations start execution.

Table 3.1 shows IOperation interface methods for supporting monitoring.

Table 3.1 IOperation interface methods.

\begin{tabular}{|l|l|}
\hline Method & Description \\
\hline $\begin{array}{l}\text { getDescription } \\
\text { getProgressState }\end{array}$ & $\begin{array}{l}\text { Returns a natural-language text description for the operation } \\
\text { Returns the amount of work done and the total amount of work } \\
\text { of the operation }\end{array}$ \\
getCurrentState & $\begin{array}{l}\text { Returns the current state of the operation } \\
\text { RetChildOperations }\end{array}$ \\
\hline
\end{tabular}

\subsubsection{ProgressState Class}

The ProgressState class represents a snapshot of the execution progress of an operation in a given instant. It provides the amount of steps done (work items) and the total steps needed for the operation to complete. These two values, together, determine the percentage of completeness of the operation.

If the operation is a composition of suboperations, then the total amount of steps needed by the operation is equal to the sum of steps needed by the suboperations, just as the amount of steps done is equal to the sum of steps done by the suboperations. Both equations must hold for a consistent progress monitoring.

The framework model does not establish fixed rules or specific metrics for determining steps of a service. ProgressState semantics are relative by comparing steps done and total steps of an operation. One might use dimensionless units. In a data transfer operation, the amount of steps could be equal to the amount of bytes transferred. In a database update operation, the amount of steps could be the number of records affected in the database. In a graph navigation operation, the amount of steps could be the number of nodes in the graph. The amount of steps executed by a service can be arbitrarily determined by the developer, by analyzing algorithms, estimating appropriate costs for blocks of code and each suboperation, according to code specificities and domain-specific analysis. The semantics used for counting steps must be regular among the operation, in order to achieve consistency.

One can inquire whether the assigned costs should be expressed in time units, representing the required duration for the operations to execute. In fact, it would be desirable. However, the actual execution duration is sensible to hardware and other context-specific characteristics. In addition, working with time units would introduce additional concerns for the management and aggregation of concurrent execution. If brick wall, under construction, is supposed to have 50 meters but it is currently with 20 meters, then we can say it is currenty on $40 \%$ of overall progress, despite the resoures involved. For this reason, although the framework does not impose intrinsic contraints for time usage, we suggest that the costs are based upon the steps needed for performing the service instead of the time durations. 
ProgressState structure defines only two members, as shown in Table 3.2. The progress percentage can be clearly determined by dividing getDoneCost by getTotalCost.

Table 3.2 ProgressState class members.

\begin{tabular}{|l|l|}
\hline Method & Description \\
\hline getDoneCost & $\begin{array}{l}\text { Returns dimensionless integer value representing the } \\
\text { number of steps already completed for the operation } \\
\text { RetTotalCost }\end{array}$ \\
$\begin{array}{l}\text { Returns dimensionless integer value representing the } \\
\text { number of steps required for the operation execution }\end{array}$ \\
\hline
\end{tabular}

\subsubsection{EOperationState Enumeration}

The EOperationState enumeration represents all possible states for an IOperation, as shown in Table 3.3

Table 3.3 Possible states for IOperation.

\begin{tabular}{|l|l|}
\hline State & Description \\
\hline $\begin{array}{l}\text { Idle } \\
\text { Processing }\end{array}$ & $\begin{array}{l}\text { Indicates that the operation execution has not started yet. } \\
\text { Indicates that the operation has started and is still running. } \\
\text { Indicates that the operation has started and is still running } \\
\text { but the consumer has requested its cancelation. } \\
\text { Indicates that the operation was running but a cancelation request } \\
\text { is being handled by the operation or the service itself started a } \\
\text { cancelation procedure due to business-specific rules. } \\
\text { Indicates that the operation has been successfully canceled in the } \\
\text { middle of execution. } \\
\text { Indicates that the operation has completed its execution successfully. } \\
\text { Indicates that the operation has been interrupted without successful } \\
\text { Interrupted }\end{array}$
\end{tabular}

Figure 3.9 illustrates the state transition diagram for the IOperation. There are 11 possible state transitions for an IOperation.

- Initial State -> Idle - When the operation is instantiated, it remains on Idle State, waiting for the execution process to start.

- Idle -> Processing - When the operation execution start is imminent, it changes from Idle to Processing State.

- Processing -> CancelRequested - When the operation execution has started and the higher-level tier requests service cancelation. This transition is a result of call to method Cancel on the IServicesMonitor.

- Processing -> Canceling - When the service provider decides to cancel the operation, despite the absence of cancelation requests, due to business-specific rules. 


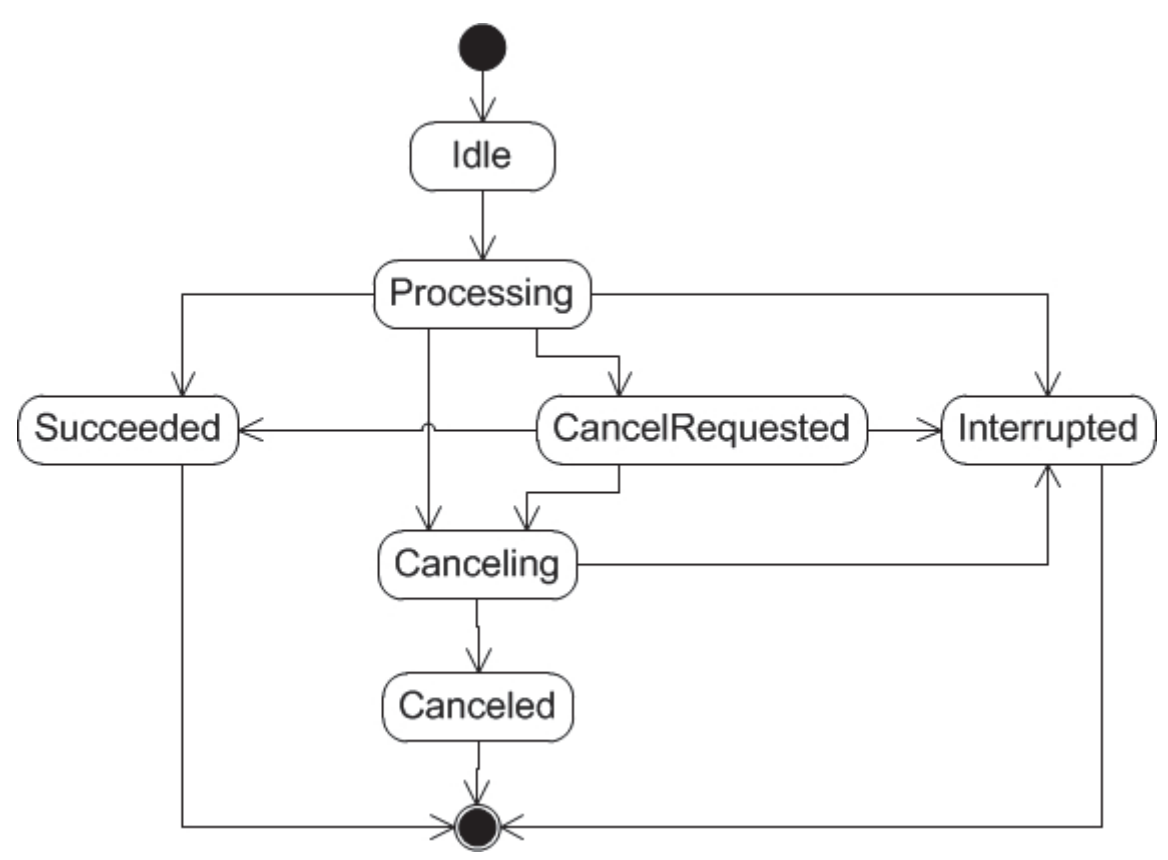

Figure 3.9 Operation state tsransition diagram.

- Processing -> Succeeded - When the operation executes completely. Once in this state, the operation remains idle.

- Processing -> Interrupted - When the operation encounters errors that require them stop abruptly. Once in this state, the operation remains idle.

- CancelRequested -> Canceling - When the cancelation request is detected and is about to get handled by the service.

- CancelRequested -> Succeeded - When the cancelation request is not handled by the service provider (commonly occurs when the cancelation request arrived too late the operation is not prepared to undo suboperations already completed). In such case, the cancelation request is simply ignored and the execution continues normally to Succeeded state. Once in this state, the operation remains idle.

- CancelRequested -> Interrupted - When the operation encounters errors that require them stop abruptly. Once in this state, the operation remains idle.

- Canceling -> Canceled - When the operation cancelation completes. Once in this state, the operation remains idle.

- Canceling -> Interrupted - When the operation encounters errors that require them stop abruptly. Once in this state, the operation remains idle.

\subsubsection{Monitoring Package}

Once the basic classes in the domain of operations and notifications have been defined, we present the monitoring interfaces that allow the consumer to access monitoring information. 
The Monitoring Package provides the main interfaces that enable consumers and providers to communicate and exchange monitoring information. Figure 3.10 presents all entities in a single diagram.

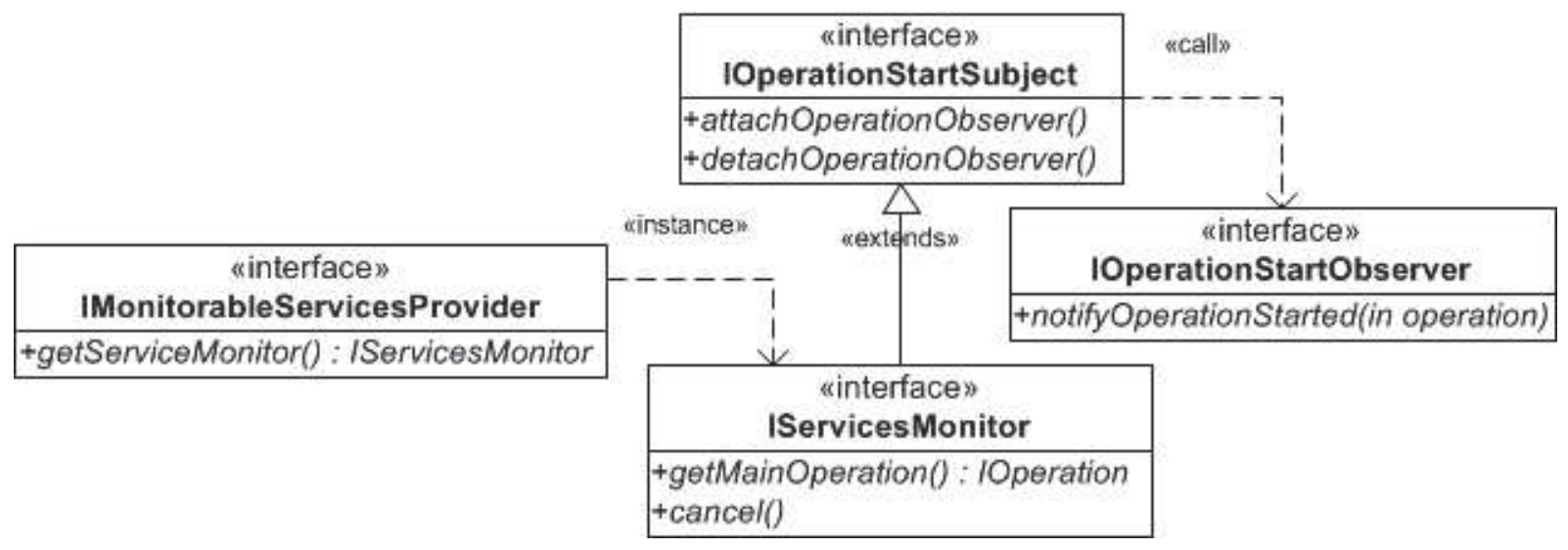

Figure 3.10 The Monitoring Package.

The main entities in the Monitoring Package are IMonitorableServicesProvider and IServicesMonitor. The IServicesMonitor interface represents the boundary between consumers and providers. Consumers receive monitoring information through an instance of IProgressMonitor. Providers also update monitoring information through an instance of the same IProgressMonitor. By using a monitor, one can register itself to be notified whenever a service main operation starts (the interface extends IOperationStartSubject). It allows accessing the IOperation instance for the main operation (by calling the getMainOperation method) and allows consumer to send cancelation requests to the running operation (by calling the cancel method).

The IMonitorableServicesProvider Interface must be implemented by every Service Provider with monitorable services. It provides an IServicesMonitor instance for the consumer to interact. For example, a class implementing a Facade contract [GE95] should also implement the IMonitorableServicesProvider interface if its services are monitorable.

Typically, there is a one-to-one relation between IMonitorableServicesProvider and IServicesMonitor. The separation is useful for modularization between business-specific code (which will be implemented for the IMonitorableServicesProvider instance) and monitoringspecific code (which will be implemented in the IServicesMonitor instance).

Once the Monitoring Package structure has been presented, we can illustrate the monitoring protocol with a sequence diagram example, as shown in Figure 3.11.

\subsection{Monitoring impact on Application Architecture}

In order to better understand how the IMonitorableServicesProvider interface must be used in practice, consider a Service Tier of a hypothetical application, which has been implemented using the Facade Design Pattern [GE95]. If we wanted to add monitoring capabilities to the Facade services by using the framework model, we should make the class implementing the facade interface also implement the IMonitorableServicesProvider interface and therefore provide an IServiceMonitor through which the consumer would access monitoring information.

In this case, we should consider two different scenarios. 


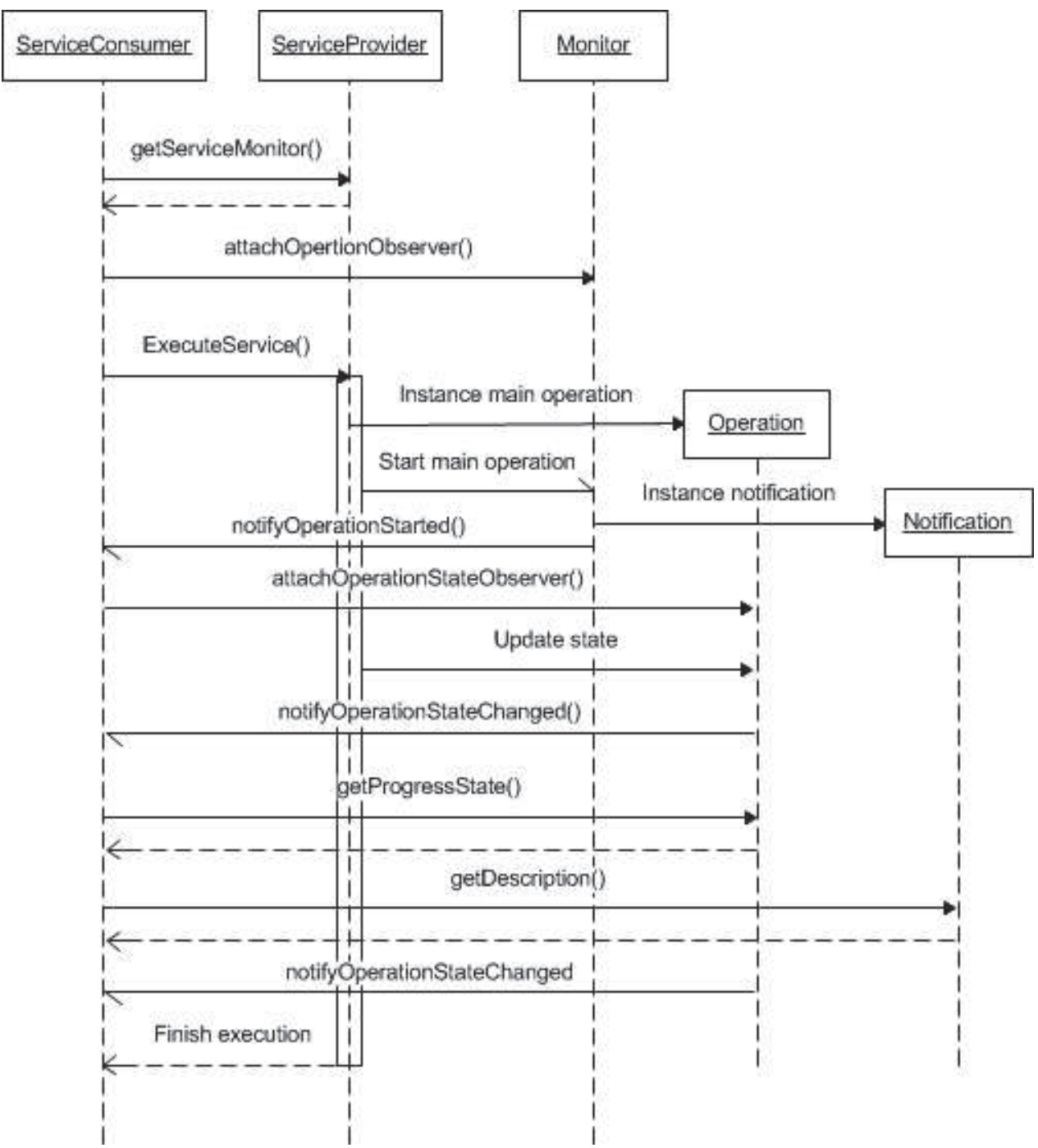

Figure 3.11 Sequence diagram example for the monitoring protocol.

- There is no support for concurrent service calls. Services provided by the facade will not be consumed simultaneously. In this case, it is possible to add monitoring capabilities with minimal architectural changes.

- There is support for concurrent service calls. Services provided by the facade can be consumed simultaneously. In this case, the application architecture might need to be slightly modified in order to comply with the framework model.

To illustrate our hypothetical application tier, consider the traditional Facade specified in Figure 3.12. The next sections describe each scenario in more details.

\subsubsection{Sequential scenario}

Considering the absence of concurrency in services execution, it is possible to have only one monitor instance for the whole facade instance. Each time a service is executed, the same monitor instance can be used by the higher-level tier to receive execution feedback. The impact on 


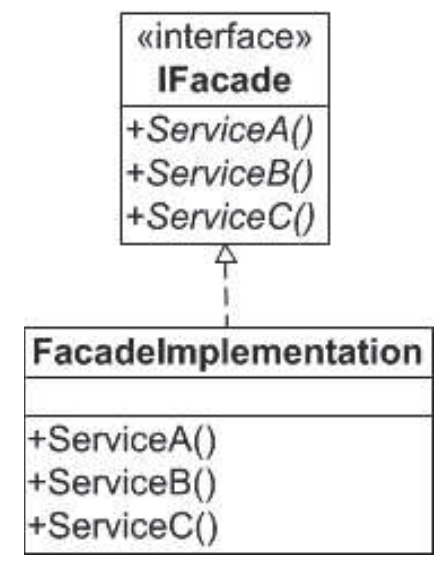

Figure 3.12 Example of typical Facade, not monitorable.

existing implementation is minimal. Figure 3.13 demonstrates the corresponding monitorable facade for this scenario.

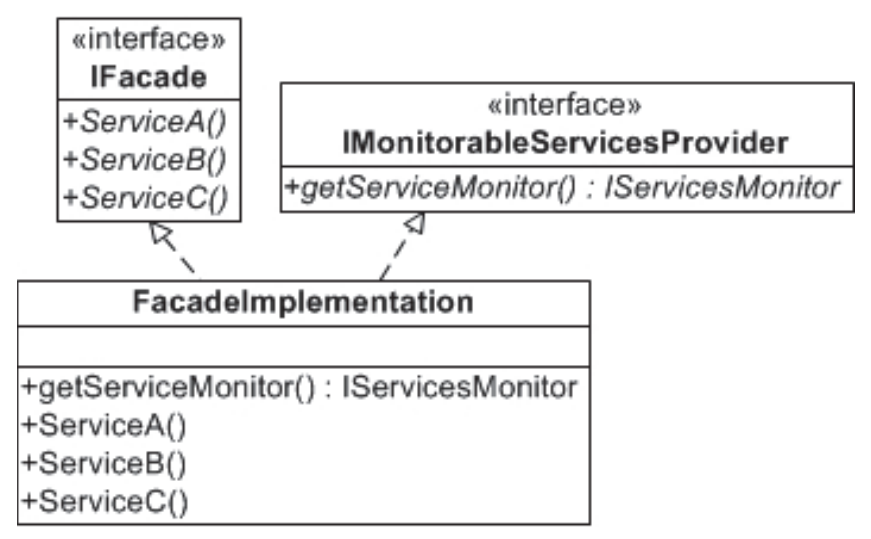

Figure 3.13 Facade adapted to extend IMonitorableServicesProvider.

\subsubsection{Concurrent scenario}

In a concurrent scenario, each service execution should have its own monitor. In this case, the application architecture should be adapted. Instead of using a Singleton Facade, we need a Facade Provider responsible for creating as many facades as the number of concurrent executions needed. Every time the consumer needs to execute a service, it will need to create a new facade by using the Facade Provider. The returned Facade will be used sequentially, so that each Facade will serve for a single execution session. This approach supports usage of one monitor per facade instance. Figure 3.14 demonstrates the adapted architecture.

\subsubsection{Adapting existing systems}

One can inquire whether it is possible to adapt an existing non-monitorable implementation into a monitorable one. The framework structures have been designed to minimize the impact on the application architecture. For example, it does not change the method signatures or the main ser- 


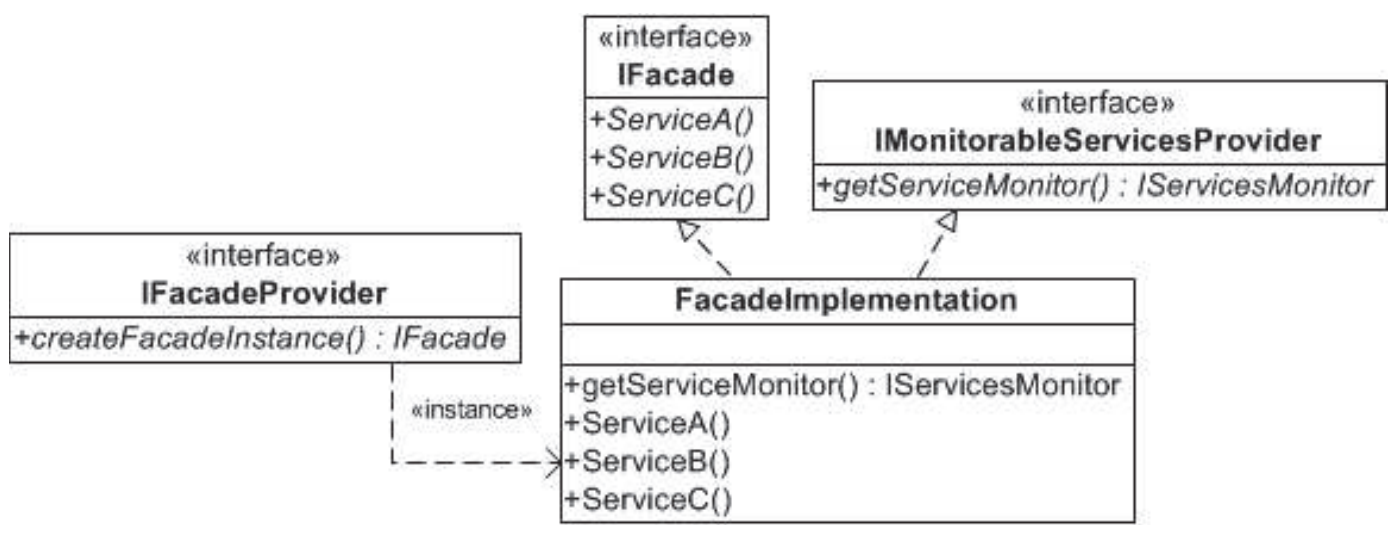

Figure 3.14 Facade adapted to extend IMonitorableServicesProvider and support concurrency.

vice facades. On sequential scenarios, it is possible to make an existing service provider class monitorable, by implementing the IMonitorableServicesProvider interface, without needing to refactor or rebuild the client consumers. Therefore, it is possible to implement monitoring $a$ posteriori. For concurrent scenarios, such as the one described in the previous section, some refactoring is required for the client consumers, since they are not able to directly access the service facade. Ideally, for concurrent scenarios, if one intends to implement monitorable services in the future, the basic architecture should be anticipated in order to avoid major refactorings.

\subsection{Chapter Summary}

In this chapter, we presented the general purpose framework base specification that provides support for building the consumers and providers specific APIs. The framework consists of three main packages that define operations, notifications and the actual monitoring interfaces. We demonstrated how operations, notifications and monitorable services are represented and how they interact with each other. It was only used object-oriented structures that conform to UML standard specification. 



\section{CHAPTER 4}

\section{Libraries}

The previous chapter presented a framework with the main classes and interface definitions for representing monitoring-related entities. In addition to class definitions, the framework establishes the protocol for consumers and providers to exchange monitoring-related data. The framework provides the skeleton for implementing monitoring, but it alone does not provide a complete solution for monitoring implementation, because it does not provide a concrete implementation for managing operation creation, its state changes and progress feedback generation. This is expected, as the framework main purpose is only to provide extendable base abstract definitions, and not a specific concrete monitoring strategy. Thus, for it to be useful in practice, one would need to extend its classes and implement the details of controlling operations, notifications and generating progress feedback.

Actually, any application could extend the framework directly and handle the operation management, chaining control, state changes, event triggering and overall progress calculation. Nonetheless, this would lead to loss of development productivity, increase of code complexity and add maintainability difficulties to the software under development. Therefore, the framework represents a fundamental step towards monitoring implementation, but it is still not enough for practical use in an application development process. In practice, a developer will certainly need the support of a concrete library, with self-contained components for handling monitoring implementation details to be encapsulated and reused throughout the application.

In this context, this chapter presents two component libraries that extend the framework by adding specific implementations for operation management: the Service Provider Library (Section 4.1) and the Service Consumer Library (Section 4.2), as illustrated in Figure 4.1.

Before we present the libraries implementation details, it is important to understand that not all the methods in the implementation classes map to the methods defined in the framework interfaces. The framework interfaces only define methods intended for the service consumer to retrieve monitoring information from the service provider. Therefore, the framework does not define secondary methods eventually needed depending on the specific strategy adopted for creating operations and notifications. In order to better understand this concept, let us suppose we are consuming monitorable services implemented by someone else. In this situation, we would not know the specific strategy used by the service provider to create its operations and notifications (for example, they could have been implemented using a different library, other than the one proposed here). For this reason, such specific methods are not exposed to the consumer through the framework interfaces, but they appear in the implementation classes presented in this chapter.

The following sections describe the libraries, presenting their classes and demonstrating examples of their usage in practice. 


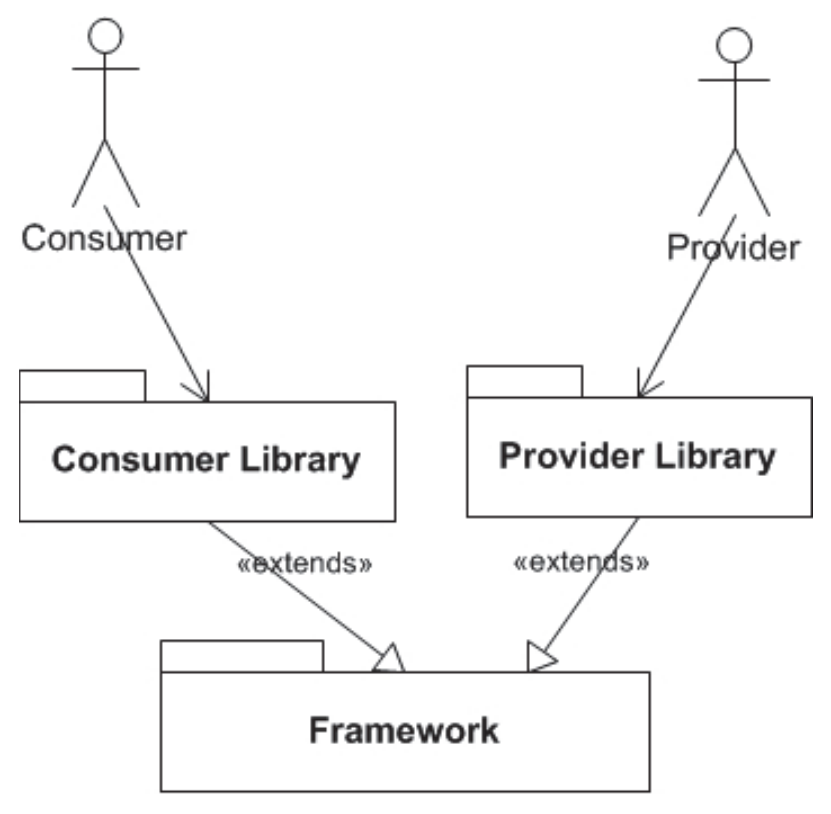

Figure 4.1 Dependencies between the component libraries and the framework.

\subsection{The Service Provider Library}

The purpose of the Service Provider Library is to provide facilities for the implementation of monitorable services, by presenting concrete mechanisms for managing operations and notifications, defined on the framework, and structures for simplifying implementation of monitoring interfaces, such as IMonitorableServicesProvider. The library provides a default lightweight logic for handling operation creation, state changes, chaining and notifications, allowing the service developer to focus on business rules and thus minimizing the impact of monitoring code on the application development complexity.

As shown in the previous chapter, for a service to be monitorable it must be hosted by a class implementing the IMonitorableServicesProvider interface and thus it must provide the consumer with an instance of the IServiceMonitor interface, in order for the monitoring information to be collected. The Services Provider Library contains classes that implement these interfaces.

\subsubsection{IDisposable Interface and using Statement}

Before we begin describing the library, let us introduce two language concepts that have proved relevant for this work: the IDisposable Interface [Lib], a native definition of the Microsoft .Net Framework, and the using language Statement, available in the C\# Language [Cor10a].

The IDisposable Interface is used to represent objects that allocate resources in memory and, after being used, should have those resources explicitly released by the developer. In general, when an object implements the Disposable Interface, the developer must notify when it has finished using it by calling method Dispose, which releases resources.

In order to ensure the call to the Dispose method, the code pattern in Listing 4.1 becomes very common: 
Listing 4.1 try-finally code pattern for releasing resources

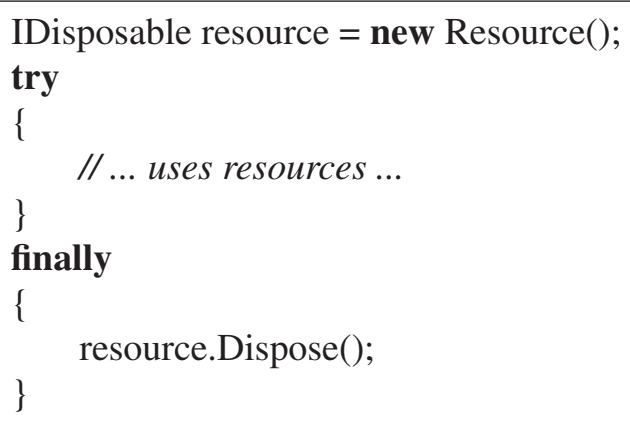

In order to make the programming source code cleaner, the C\# Programming Language provides the specific statement using for dealing with Disposable resource consumption, eliminating the need of writing too much try-finally blocks throughout the application source code. With this statement, the previous example could be rewritten as in Listing 4.2.

Listing 4.2 using code pattern for releasing resources

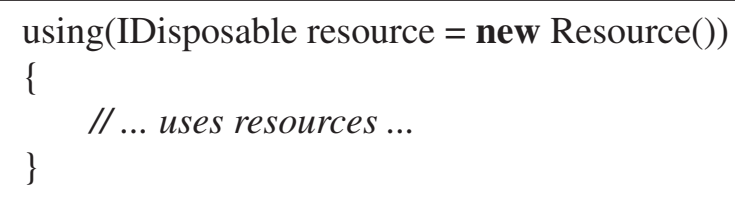

The previous example has the same effect as the one that uses the try-finally construct, but the call to tbe Dispose method is implicit. In the next section, we explain how to make use of this facility of the $\mathrm{C \#}$ Language in the context of the monitoring framework.

Although we illustrate the benefit of using a C\# specific language construct, all the library model is fully compatible with Java or any other object-oriented language providing only standard language statements. The using statement is easily convertible into a try-finally statement and it is used for the convenience of reducing the amount of lines of code.

\subsubsection{ServiceMonitor class}

The ServiceMonitor class is the central element of the library. It implements the IServiceMonitor interface and allows the developer to create operations and notifications that will be seamlessly exposed to the service consumer.

Any class exposing monitorable services — such as a Facade of a typical application layer — should have one instance of a ServiceMonitor in order to create operations. Figure 4.2 shows the ServiceMonitor class members.

Most of the ServiceMonitor class members are a result of implementing the IServiceMonitor interface. The getMainOperation and cancel methods come directly from the IServiceMonitor interface, while the attachOperationObserver and detachOperationObserver methods come from the IOperationStartSubject interface. Since the IServiceMonitor extends the IOperationStartSubject interface, the ServiceMonitor class must provide implementation for the methods of both interfaces. These interface methods are described in Section 3.2.4.

In addition to the methods defined in the framework interfaces, the class defines the method createOperation which must be called in the beginning of the service execution in order to create the very first operation, which will become the monitor main operation. Once this method is called, a new operation is created and the monitor state changes from Idle to running. It 


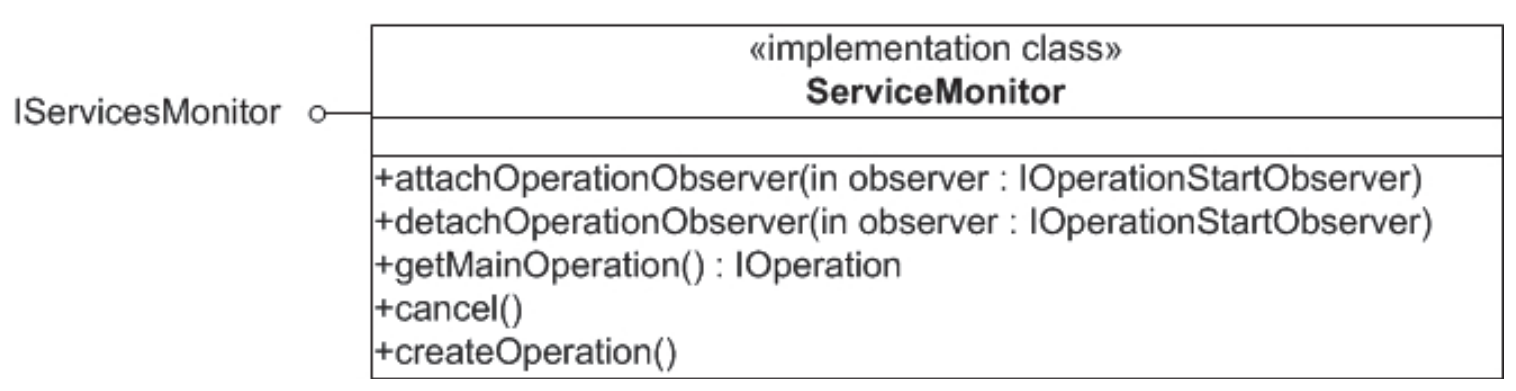

Figure 4.2 ServiceMonitor class members.

should not be called again until the operation is finished, either by succeeded completion or by cancelation or error, otherwise the monitor will raise an exception. This occurs because the monitor implementation assumes no concurrent calls in the same monitor. Thus, it does not allow for two different consumers to share the same monitor for different execution contexts. If an application requires concurrent executions, it must use a design pattern to separate the monitor instances and isolate contexts, such as shown in Section 3.3.2.

Although there must exist only one single main operation per service call, the main operation can be logically split into suboperations, each one contributing to the execution of part of the whole service execution. There is no limit for the amount of child operations created while the service executes. After creating the main operation by calling method createOperation, one can control operations by interacting with the CustomOperation class, which is described in the next section.

\subsubsection{CustomOperation class}

The CustomOperation class is the concrete representation of the operation concept and thus implements the IOperation interface described in previous chapter.

Figure 4.3 shows the CustomOperation class members.

Most of the class members are an immediate consequence of implementing IOperation, IOperationStateSubject, IOperationStartSubject and INotificationsProvider framework interfaces, which were already described in the previous chapter, in Sections 3.2.3.1 and 3.2.2.1.

In addition to the methods defined in the framework interfaces, which focus on the interaction between consumer and provider, the class defines some other methods that allow controlling the operation progress state, registering notifications and creating suboperations. These additional methods are introduced because of the specific monitoring strategy adopted by the Service Provider Library, which is to allow the explicit manipulation of operations and notifications, in the context of the service provider. Table 4.1 briefly describes these methods.

Besides explicitly controlling progress state and registering notifications, the CustomOperation class provides the method createChildOperation, which enables creating suboperations that binds to the parent operation in such a manner that changes to the progress state of a child operation recursively update all the chain of parent operation progress states.

In this context, the library provides three different methods for dealing with suboperations. They are briefly described as follows.

- Freely create and update child operations. This is the most basic method for creating child operations. It is also the most generic and serves any situation, suppporting both 


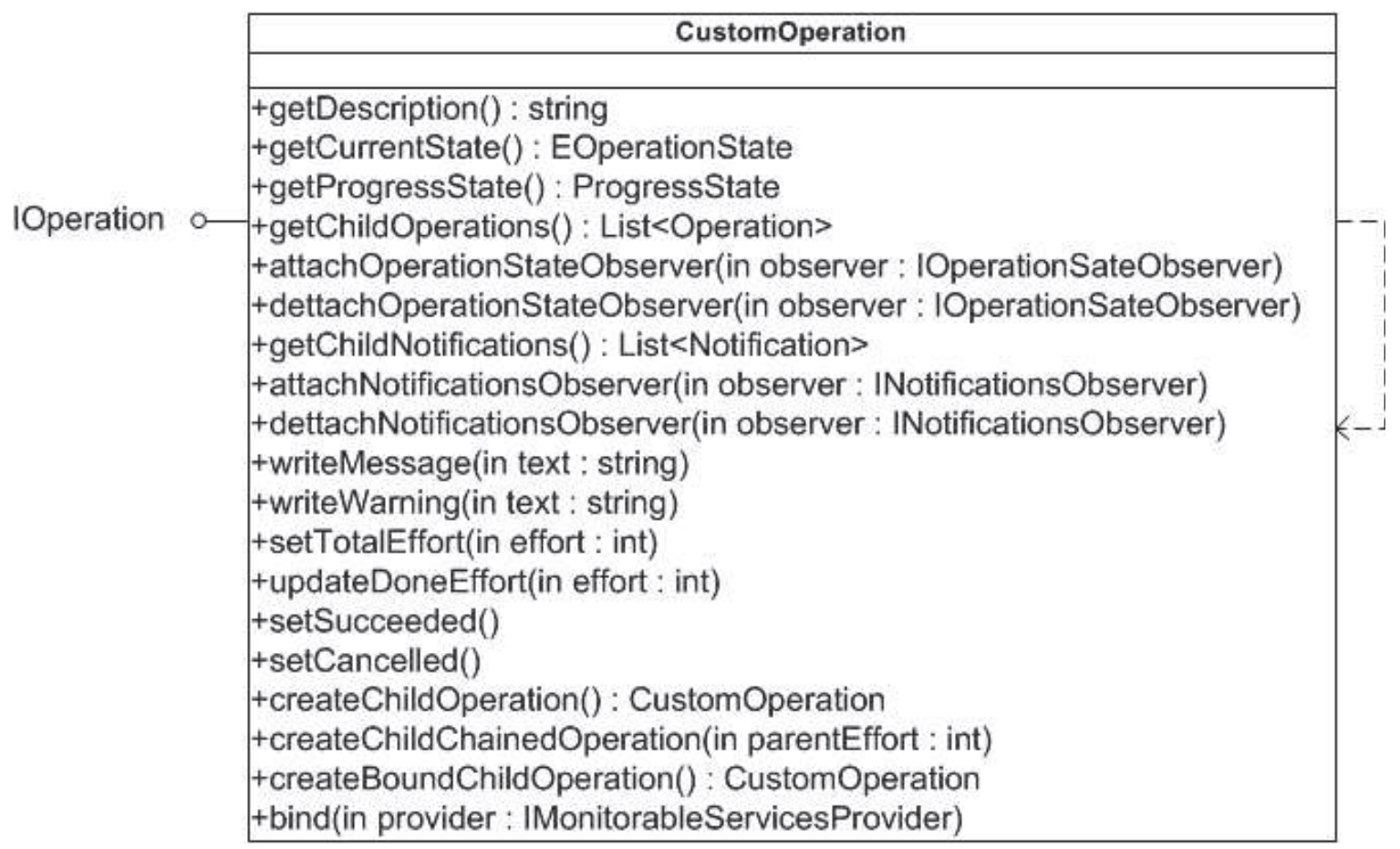

Figure 4.3 CustomOperation class members.

sequential and concurrent execution contexts. The developer needs to explictly manage the progress updates for each child operation created.

- Bind to another service sharing the same monitor instance. This method for creating child operations is a facility for a specific situation, when the suboperation runs as a chained execution of another service sharing the same monitor (possibly another method in the same instance). Since they share the same monitor instance, it is possible to automatically bind the second service executed as a suboperation of the first service executed. This method can only be used in a sequential execution context.

- Bind to another service with its own monitor instance. This method for creating child operations is a facility for a specific situation, when the suboperation runs as a chained execution of another service with its own monitor (possibly another method in another instance). Although they do not share the same monitor instance, it is possible to explictly bind both monitors so that the progress updates of the second one scales to the first one as a suboperation. This method can be used in both sequential and concurrent execution contexts.

Although each situation has its own peculiarities for the developer to deal with, the library provides a regular code pattern that makes it easy for the developer to handle each case. The next subsection describes a code pattern that applies for every public method representing a monitorable service of any service provider implementing IMonitorableServicesProvider. In the sequence, we show how this code pattern applies for each suboperation handling method. 
Table 4.1 CustomOperation class additional members.

\begin{tabular}{|c|c|}
\hline Method & Description \\
\hline writeMessage & $\begin{array}{l}\text { Allows creating business-specific general messages about the } \\
\text { execution state of the service. }\end{array}$ \\
\hline writeWarning & $\begin{array}{l}\text { Allows creating business-specific warning messages about the } \\
\text { execution of the service, such as errors. }\end{array}$ \\
\hline setTotalEffort & $\begin{array}{l}\text { Specifies the total amount of steps needed for the operation } \\
\text { to be fully completed. This method is usually called in the } \\
\text { beginning of the operation execution. }\end{array}$ \\
\hline updateDoneEffort & $\begin{array}{l}\text { Specifies the amount of steps already completed. This method } \\
\text { must be called as the service executes in order to update the } \\
\text { number of steps completed. }\end{array}$ \\
\hline setSucceeded & $\begin{array}{l}\text { Allows changing the operation state from Processing to } \\
\text { Succeeded. }\end{array}$ \\
\hline setCanceled & $\begin{array}{l}\text { Allows for acknowledging the pending cancelation request } \\
\text { and setting the operation state to Canceled. }\end{array}$ \\
\hline createChildOperation & $\begin{array}{l}\text { Allows creating new child operations that attaches to } \\
\text { the operation and contributes to its overall execution. }\end{array}$ \\
\hline createChildChainedOperation & $\begin{array}{l}\text { Prepares the monitor for a new child operation created in an } \\
\text { independent method context and attaches it to the operation. }\end{array}$ \\
\hline createBoundChildOperation & $\begin{array}{l}\text { Allows creating a new child operation which binds to another } \\
\text { monitor of a distinct service provider. }\end{array}$ \\
\hline bind & $\begin{array}{l}\text { Allows explict binding of the current operation to a monitor } \\
\text { of a distinct service provider. }\end{array}$ \\
\hline
\end{tabular}

\subsubsection{A Code Pattern for monitorable services implementation}

It has been explained that classes exposing monitorable public methods should implement the interface IMonitorableServicesProvider and thus provide an instance of IServiceMonitor, which is implemented in the Service Provider Library by the ServiceMonitor class.

The ServiceMonitor class controls the class monitoring state and becomes a central point for knowing whenever a new operation starts and finishes. For this reason, the only way of creating the Main Operation for a service is by calling createOperation on the ServiceMonitor. Then, by observing events of state changes in created operations, the ServiceMonitor class maintains the operations overall progress states of the class as a whole.

In this context, we present the main Code Pattern for implementing every monitorable service in the application, as illustrated in Listing 4.3. This Code Pattern will show itself very important to support specific monitoring contexts described in next sections.

Listing 4.3 Main Code Pattern for monitorable services implementation

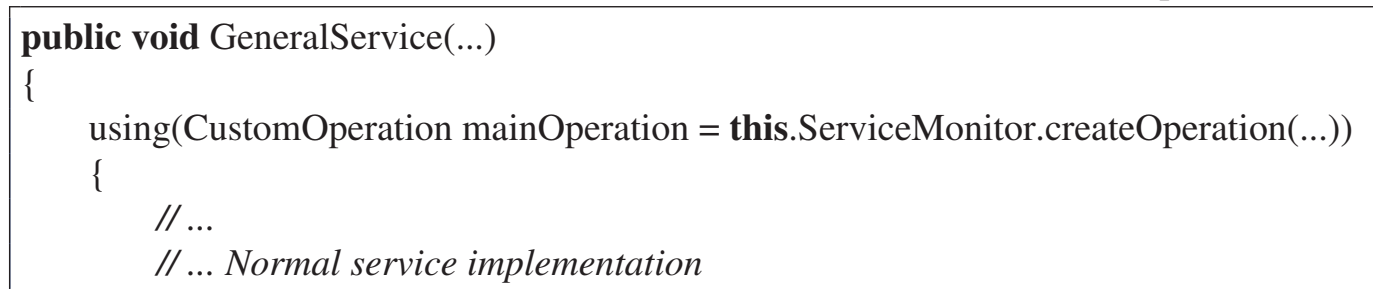


$\quad / / \ldots$
$\quad\} \quad$ mainOperation.setSucceeded();

The first line of the example in Listing 4.3 declares a fictitious public method, with zero or more input parameters. The number of parameters of the method is irrelevant for our purposes and does not affect the Code Pattern. The method signature itself is not relevant at all. This is a very important characteristic for our code pattern: it does not affect the software architecture. It only affects implementation details of the method. Thus, one could turn a non monitorable method into a monitorable one without making changes to method signatures or to the system architecture. There is no need to refactor and rebuild dependent system components in higherlevel layers. The Code Pattern begins and ends all inside the monitorable method. One can inquire whether the code pattern still contains tangled code, since the business implementation interlaces the monitoring-related code. In fact, the code pattern does not eliminate the code tangling, but it introduces a standardized and regular way of implementing the monitoring code.

The using construct is the Code Pattern itself. It creates the Main Operation for the monitorable service to report progress state and provide feedback regarding the service execution. For our current purpose of explaining the general Code Pattern for creating the service Main Operation, with no assumptions or previous knowledge regarding service-specific characteristics, it is not relevant to explain the parameters required for the createOperation call on the ServiceMonitor, where it should be provided a description for the operation and a number of steps (which represents the total effort required for the operation to be completed).

As explained in Section 4.1.1, the using Statement assures that the operation, once started, will be terminated (whether successfully or not). The last line of code before the end of the using statement is a call to setSucceeded method. If this line of code is executed, it means that all the service implementation executed normally without throwing unhandled exceptions. In this case, the operation will change its state to Success even before the using Statement disposes the main operation. On the other hand, if the operation is disposed before its state gets set to Succeeded, as a result of unhandled exception throwing, the operation state is automatically set to Failed state.

For now, this is the basic information needed to understand the logic behind creating operations in monitorable services. More context-specific details are provided in the next sections.

\subsubsection{Freely create and update child operations}

In the previous chapter, Section 3.1, we introduced the concept of operations and how a higherlevel service could be logically split into two or more lower-level operations. The higher the operation level, the higher its potential for a large chain of suboperations execution. This section discusses the most generic method for managing suboperations: by freely creating and updating their progress state.

Let us consider, for didactical purposes, that we are going to implement a monitorable service that inserts a new user in a database. In order for this service to accomplish its task, it needs to perform some basic operations: (1) connect to the system database; (2) validate if there already exists a user with the same login; (3) effectively insert the new user into the Users database table; and (4) close the database connection. 
Let us consider these four basic steps needed to perform the main operation (the user insertion) as an appropriate logical division for the suboperations. To simplify our example, let us consider a structured service implementation, and all the four operations implemented within the same method, having each operation clearly delimited by a range of code.

Before we begin, we need to provide an estimate execution "cost" for each of these steps. These "costs" determine the weight of each suboperation in determining the Main Operation overall progress state. In later sections, we discuss more details on how these "weights" could be more appropriately determined. For now, let us simply guess their values. In a Main Operation with total cost of 100 , let us consider a cost of 20 equally divided between suboperations (1) and (4), thus each one with a weight of 10 . Let us consider 40 for each of the remaining suboperations (2) and (3).

One can inquire wheter it is always a simple task to plan and determine the total execution cost. Indeed, it can be very hard to determine the total execution cost in the beginning of a service execution. For more dynamic algorithm implementations, such as graph navigation or backtracking algorithms, the cost of calculating how many iterations will be needed can be as complex as the execution itself. In such cases, one can find a limit superior for the total execution cost (and adjust it during the execution). For now, let us assume simple costs for the operations.

Once the suboperations and their costs are planned, we are prepared to implement our monitorable service, as in Listing 4.4.

Listing 4.4 User insertion monitorable service

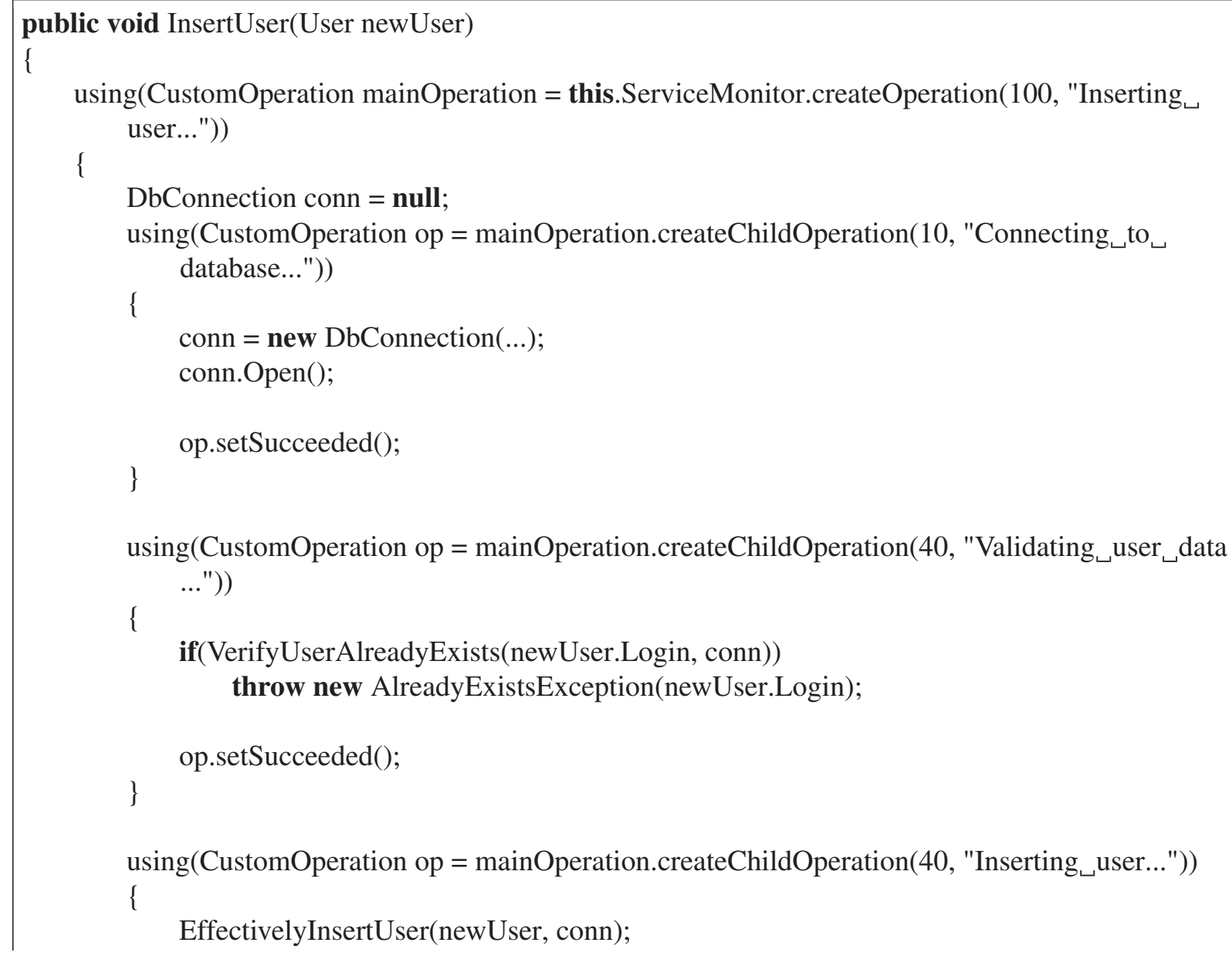




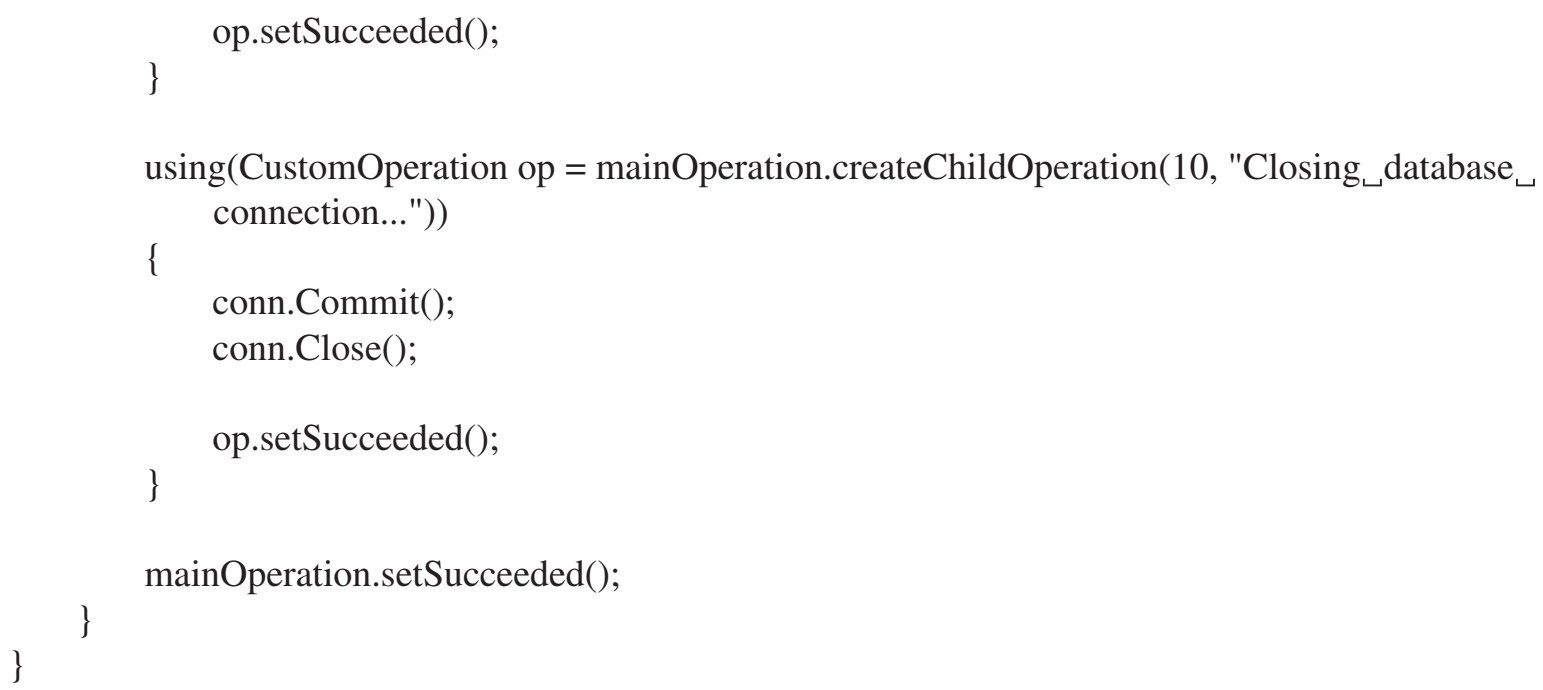

The same code pattern applied to the Main Operation creation also applies to its suboperations. The call to setSucceeded on each suboperation fires a cascading update effect on every parent operation, automatically updating their progress state and asynchronously sending feedback to attached observers. Suboperations may also contain other suboperations. There is no limit for the depth of operations tree (or suboperations subtree).

In a concurrent operation execution context, the principle of creating operations and calling setSucceeded method upon execution completion remains the same, but it adds an additional concern: since the code execution is not sequential, the developer would need to add specific code to wait for the asynchronous execution to actually complete, before calling the setSucceeded method. In such case, the generic code pattern explained in Listing 4.3 could be rewritten as in Listing 4.5 .

Listing 4.5 Code pattern adapted to concurrent execution

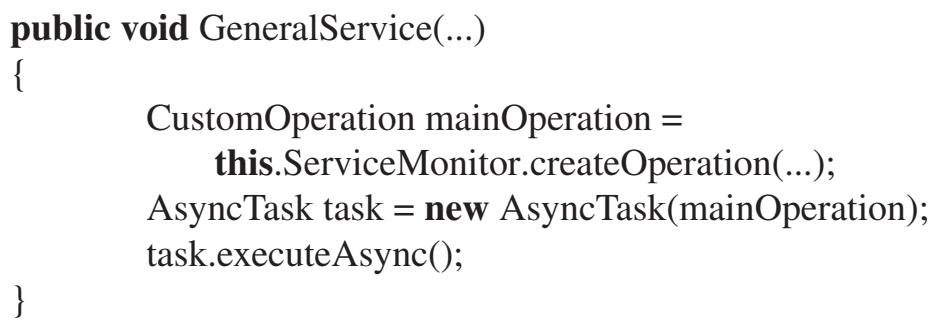

In the adapted pattern shown in Listing 4.5, the actual business-specific code has been isolated within a fictitious class named AsyncTask, which is parametrized by the mainOperation. It is up for this class to call the setSucceeded on the operation instance, upon the execution completion. The using pattern also could not be used, since the execution runs in a different thread and the exceptions thrown in the actual execution thread would not be caught. Again, it is up to the AsyncTask class to call the Dispose method on the operation in case of unhandled exceptions are thrown.

Therefore, in order to make all the four operations of the original example in Listing 4.4 concurrent (only for illustration), we would need to adapt each one of the inner using blocks to a code similar to the one shown in Listing 4.5 , by creating a task class to isolate each asynchronous operation. The outermost using block for the main operation does not need to be 
adapted. Since the concurrent operations are bound to the main operation, the call to setSucceeded method on the main operation has an implicit logic to wait for all child operations to complete execution.

\subsubsection{Bind to another service sharing the same monitor instance}

According to [Boe97] and [PM07], and in fact it is clearly observable on the software industry, software reuse is a largely adopted technique for its potential on improving quality, maintainability and reducing projects costs.

In fact, the ability to create procedures and functions that add modularity and stimulate reuse plays a very important role in the evolution of software development.

That said, we simply cannot ignore the fact that the implementation of an operation might perform calls to several other methods that may have their own tree of operations and suboperations, cost weights, and may themselves be considered autonomous services (called directly by a higher-level layer) or at the same time be used as a suboperation of more than one method.

This section describes how the Service Provider Library deals with this very common situation.

Consider the previous example but this time with a different implementation strategy: suppose that steps (1), (2) and (4) were implemented as fully autonomous methods that can be reused as part of any other method.

The implementation of our InsertUser method could be rewritten as in Listing 4.6, without loss of suboperations creation.

Listing 4.6 Monitorable service with suboperations as auxiliary methods on the same class

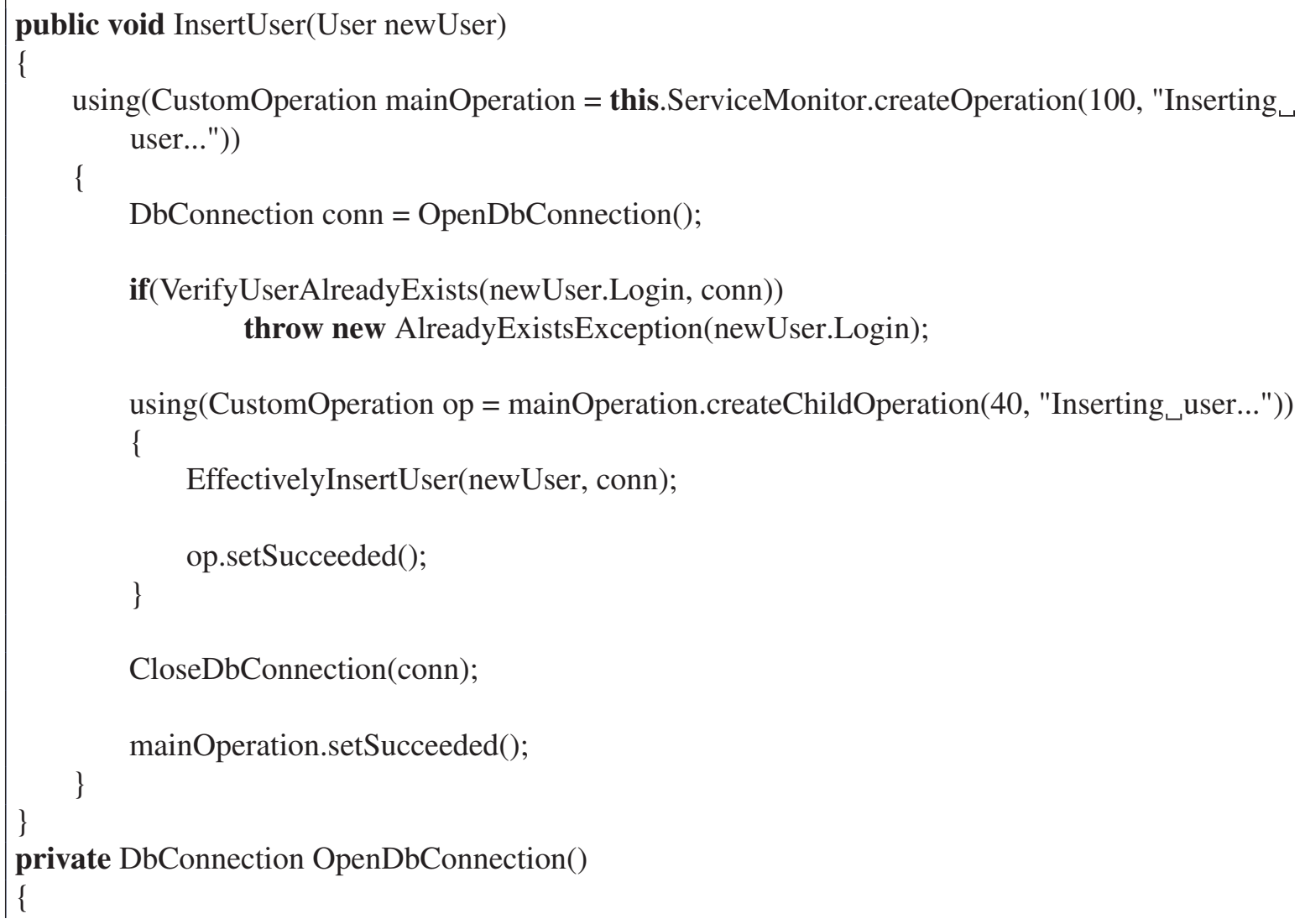




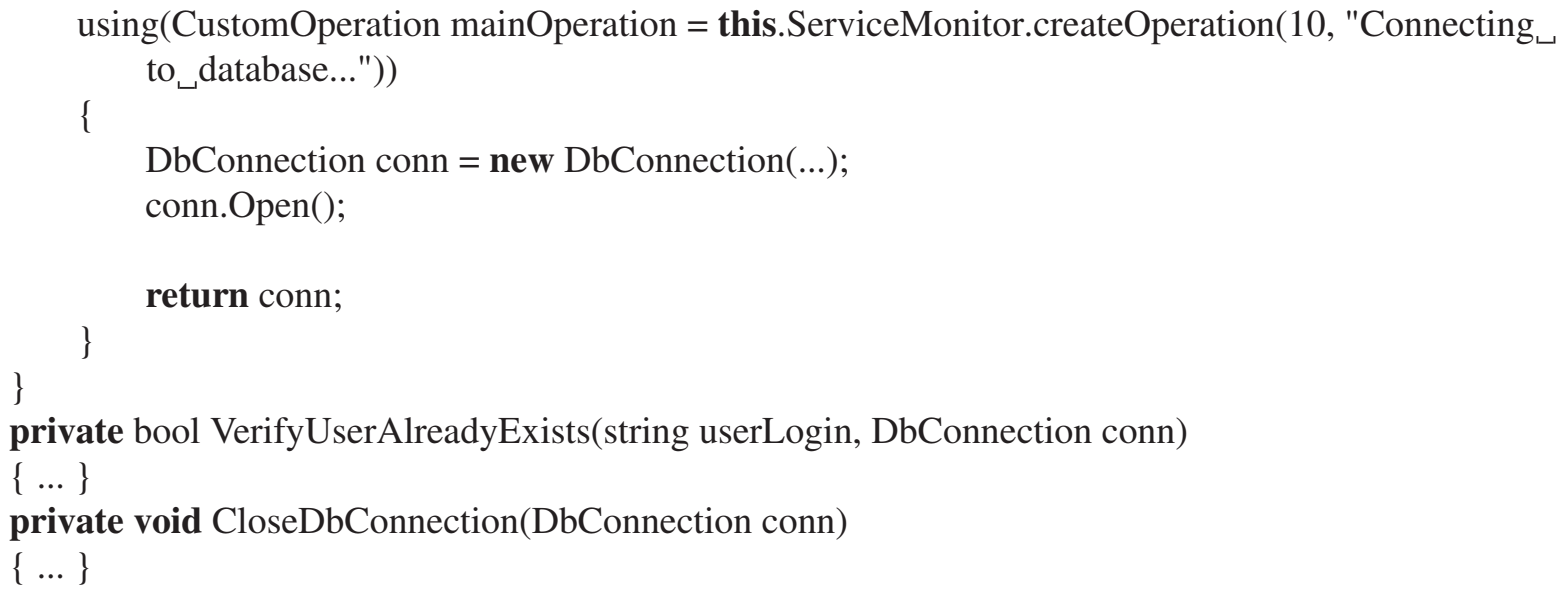

One could possibly inquire whether it would not be necessary for the OpenDbConnection and other methods to receive a reference to the Main Operation created within the InsertUser method. If we had chosen so, once again we would break the principle of not making changes to method signatures and minimizing impact on system architecture. To avoid passing operation references as parameters, we considered that if another call to the method createOperation is performed and the first call has not finished execution yet, it is automatically assumed that the new call is a suboperation of the first call. We can only make this assumption because we assume no concurrent calls are made to the same service provider, as assured by the adoption of the Design Pattern described in Section 4.1.2, which presents the ServiceMonitor class. If we did not make that restriction, we would not be able to distinguish between different callers to the same methods: some calls should be considered as suboperations and others as independent operations. We chose to add this restriction because it can be easily solved: if one wants to enable concurrent calls to the same service, an approach is simply to create new instances of the same service provider, and use these different instances concurrently. In any case, if one really needs to make concurrent calls on the same Service Provider instance, the solution is simply to ignore the principle of not changing method signatures and pass the operation references as parameter. In this case, our example should be rewritten as in Listing 4.7.

Listing 4.7 Monitorable service with suboperations as auxiliary methods on the same class and support for concurrent calls

public void InsertUser(User newUser)

\{

using $($ CustomOperation mainOperation $=$ this.ServiceMonitor.createOperation(100, "Inserting user..."))

\{

DbConnection conn $=$ OpenDbConnection $($ mainOperation $)$;

if(VerifyUserAlreadyExists(mainOperation, newUser.Login, conn))

throw new AlreadyExistsException(newUser.Login);

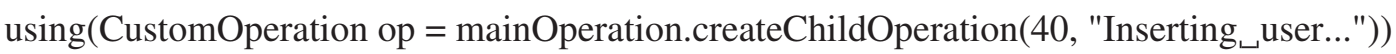

\{

Effectivelyinsertuser(newUser, conn);

op.setSucceeded(); 


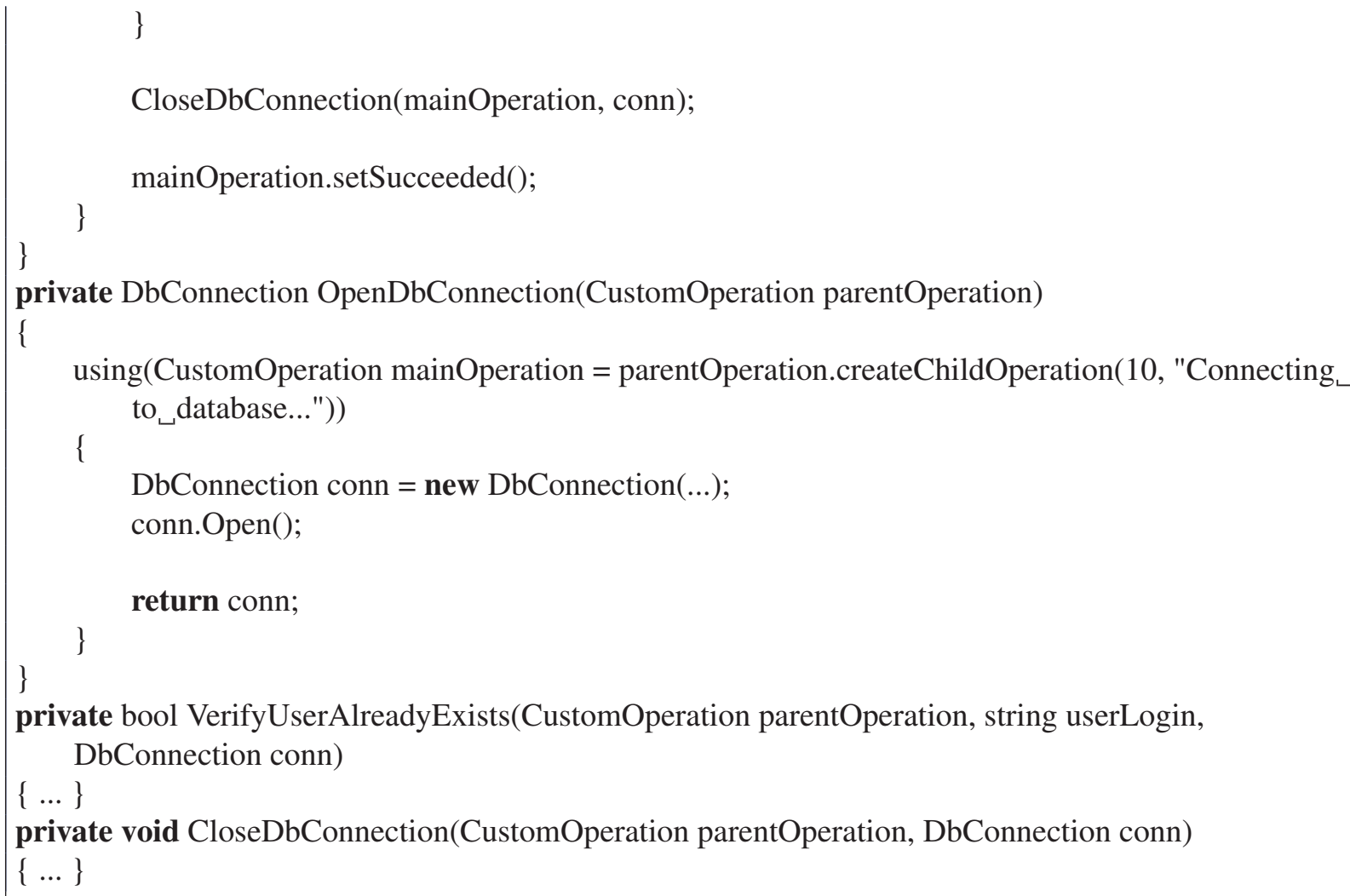

Another possible inquiry is whether it would be appropriate to use a fixed weight for an operation without making any kind of assumptions about the context in which it will be used. For example, from the moment we created a separate method for OpenDbCommand, we should give it a general implementation that does not make any assumptions about the context where it might get called. By the time we have separated the suboperation in a different method and continued to use a fixed weight of 10 for the operation, we assumed a uniform weight definition for all the operations in the Service Provider class. Alternatively, one would want to parametrize the cost of the main operation in order to make it adaptable to different scenarios and different metrics for the weight of operations. If so, the method OpenDbConnection should be rewritten as in Listing 4.8 .

Listing 4.8 Monitorable service with parametrized execution cost

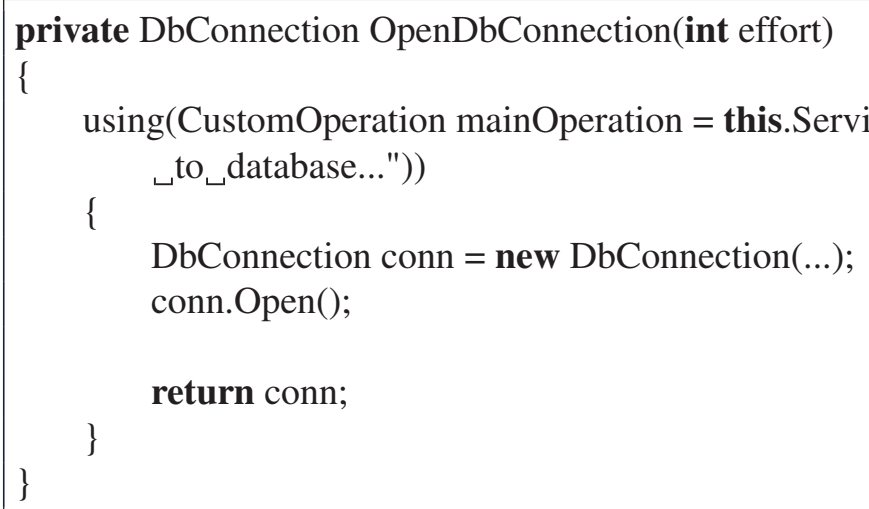

Although this change has solved the problem of creating a context-independent method, again it would not be desirable because it would break the principle of not affecting natural 
method signatures and minimizing impact on application architecture. To deal with this, we created a special kind of operation that scales the original operation effort weight into another weight in the caller context. By doing this, the method OpenDbConnection can get back to its parameterless state, and use whatever weight it considers appropriate in its own context. And the caller should, in order to avoid making assumptions about the uniformity of the operation cost weight of the method being called, explicitly define the desired cost of the operation about to be called. Thus, we should rewrite the InsertUser method as in Listing 4.9.

Listing 4.9 Defining weight of suboperations

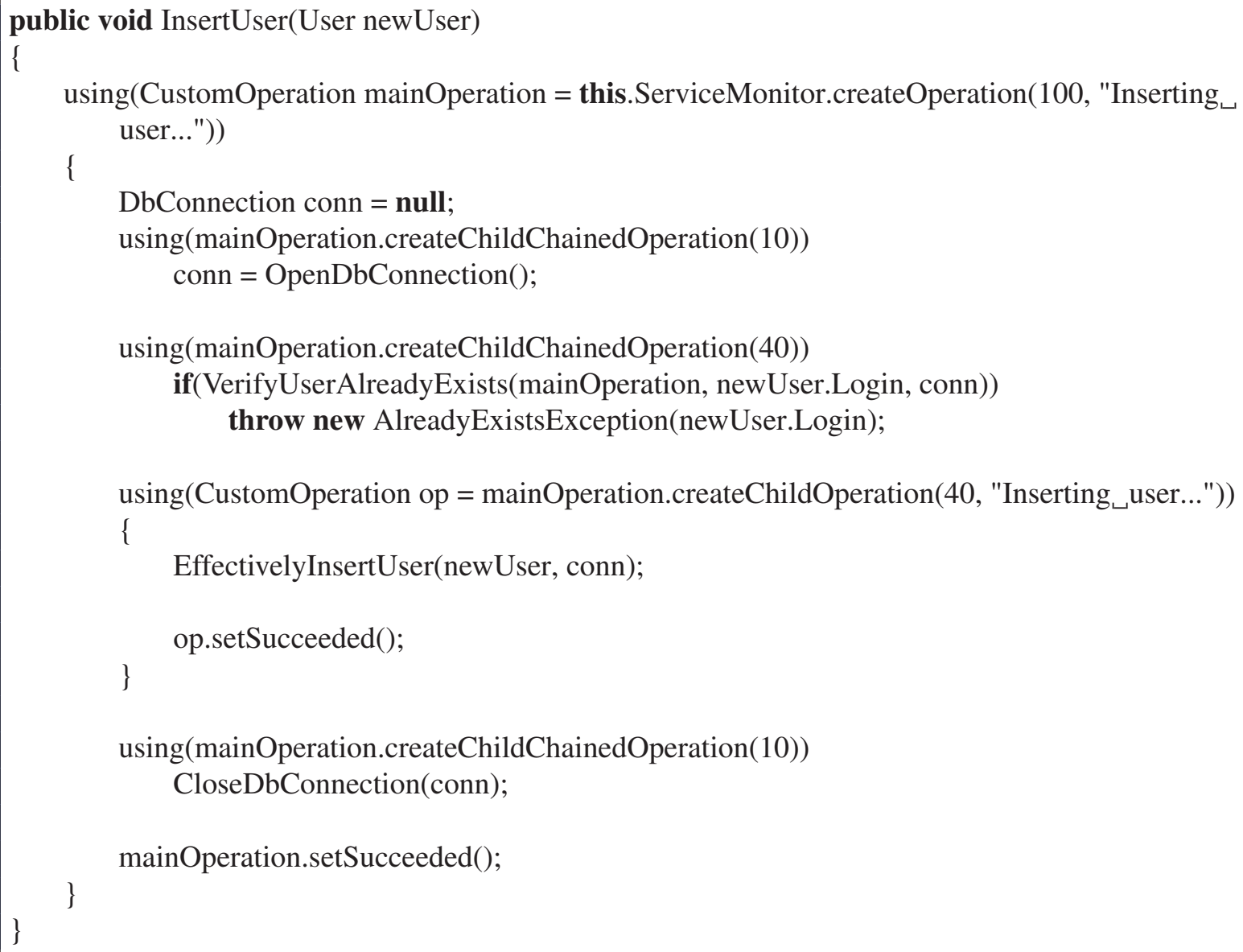

As one might have noticed, we introduced a call to createChildChainedOperation before each operation. It makes the Service Monitor wait for the next main operation to be created. Once created, it is automatically attached as a suboperation and associated with a scale-factor that converts its progress state into a weight proportional to the one desired in the caller context and thus avoiding distortions in the progress state calculations. It is not necessary to define a description or calling setSucceeded when calling createChildChainedOperation, for it will be assumed from the Main Operation created in the method being called. Again, the use of method createChildChainedOperation assumes no concurrent calls to the same Service Provider is allowed. Otherwise, we would not be able to distinguish between calls from other contexts and should not be able to attach the main operation to the correct caller context. If concurrent calls are needed for the same Service Provider instance, it should never use createChildChainedOperation and the desired effort weight for the operation would need to be parametrized for each method call. 


\subsubsection{Bind to another service with its own monitor instance}

Besides performing calls to different methods provided by the same service provider instance, it is also a very common situation to perform calls to methods provided by a variety of distinct services provider instances. In fact, it is a crucial requirement for any object-oriented modeling of service implementations, because service implementations are often a composition of services implemented on other service provider instances. This section demonstrates how to deal with this situation by using the service provider library.

The bind method, provided by the CustomOperation class, allows operations performed by one service provider instance to bind themselves to operations created in different provider instances. Again, once two operations of different contexts are bound one another, the original operation weight is proportionally scaled to a relative weight on the caller context. Also, when a parent operation binds to a child operation, it listens (through the Observer Pattern) to the child operation progress updates and scales the suboperation progress percentage to update its own progress state.

To make it clear, let us adapt our previous example: instead of implementing OpenDbConnection and CloseDbConnection on the same service instance, let us consider a separate class for managing a database connection pool that might be used by several other services in different class instances.

In this case, Insert User becomes a method of the UserServicesProvider class, while OpenDbConnection and CloseDbConnection becomes a method of the DbConnectionServicesProvider class.

The InsertUser method of UserServicesProvider class should be rewritten as ìn Listing 4.10.

Listing 4.10 Executing suboperations in distinct provider instances

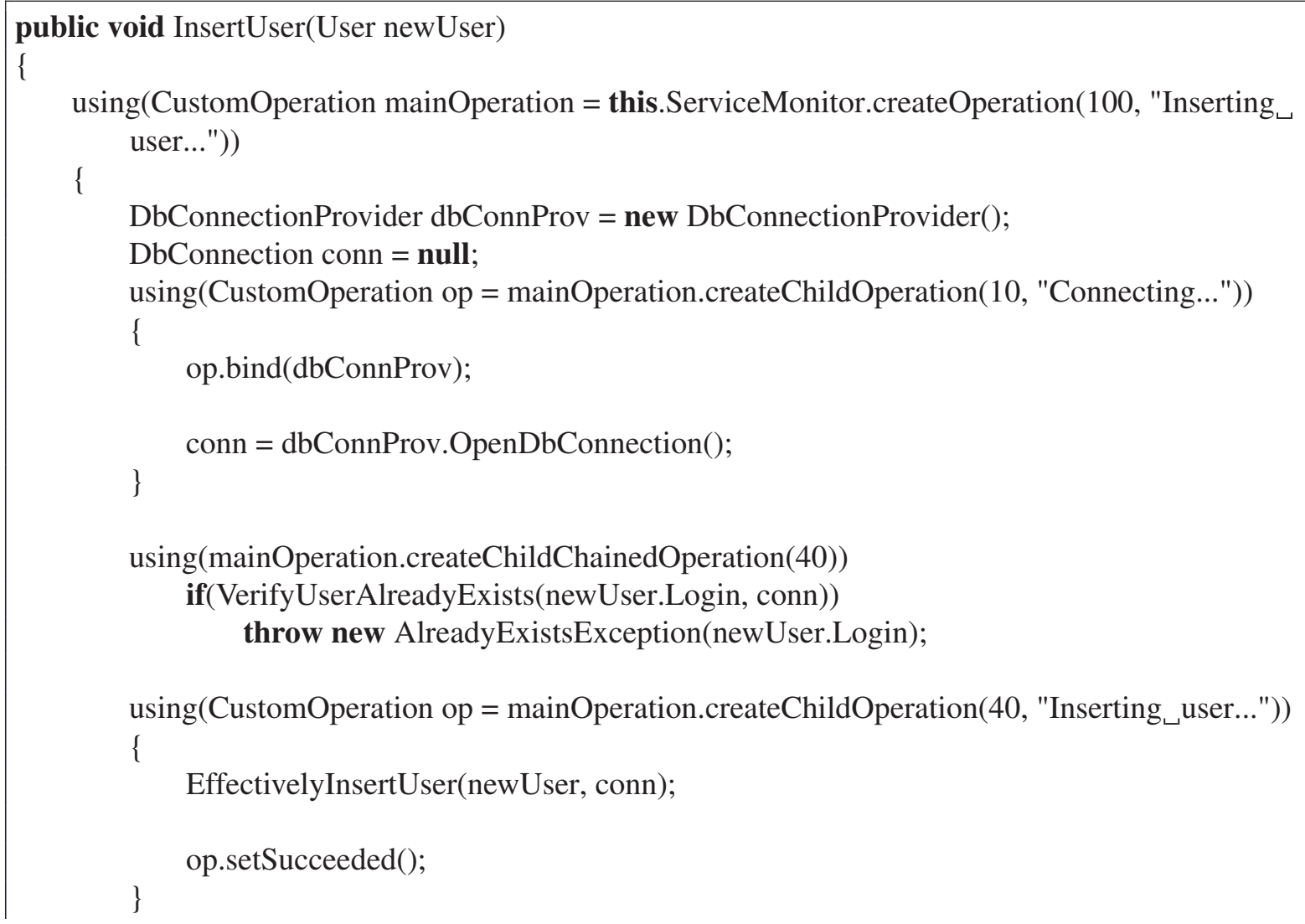




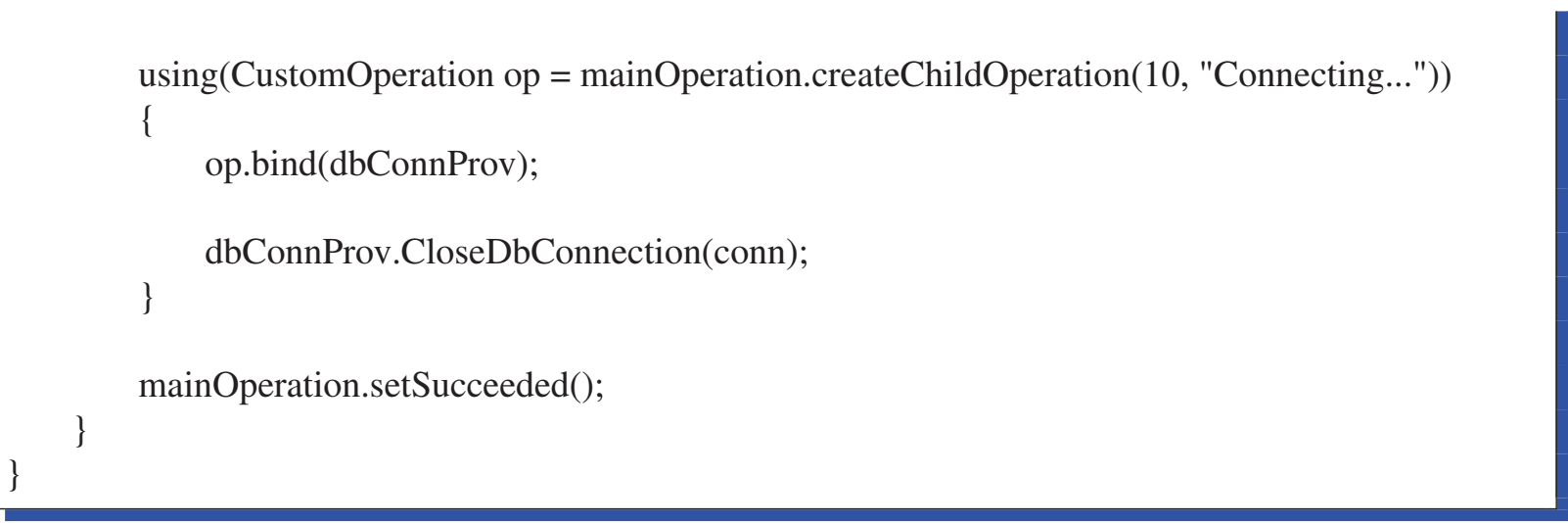

The OpenDbConnection method should remain the same, but implemented as a member of the DbConnectionProvider class.

In order to simplify code and suppress the need for calling the bind method, the library also provides a specific method for creating child operations that bind to another IMonitorableServiceProvider. Thus, we could rewrite the code that opens database connection as shown in Listing 4.11.

Listing 4.11 Creating child operations that binds to another IMonitorableServicesProvider

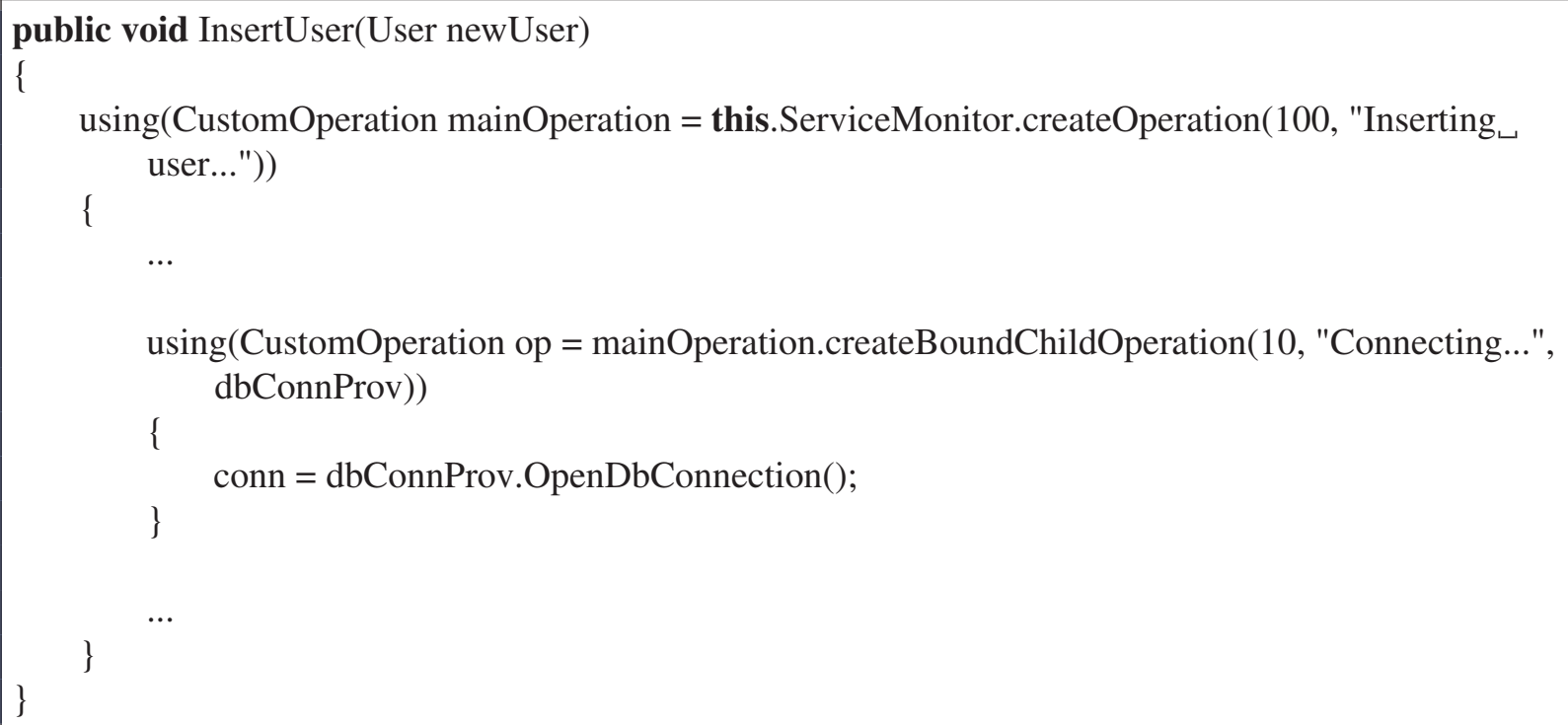

Note that the operation binding is performed before the child operation actually starts execution. By the time a call to method OpenDbConnection is performed on DbConnectionProvider instance, it creates its own main operation inside its own monitor. Thus, in fact, we have two operations being created: the main operation for the DbConnectionProvider context, and a child operation in the UserServicesProvider context. These two operations are independent but still they are bound so that progress updates notified by the main operation in DbConnectionProvider causes progress update effects on UserServicesProvider child operation.

\subsubsection{Recursive Operations}

As it has been shown in previous examples, every operation created within a monitorable service execution context has an associated cost. The logic behind constructing isolated and independent operation contexts allows a service consumer to completely abstract the nature and 
complexity of the consumed services. Thus, despite the complexity or length of the operation execution chain, the developer might be able to abstract execution contexts that rely within a called suboperation, knowing that progress state updates will get scaled along the chain and automatically and seamlessly update its own progress state.

On sequential execution contexts, this facility is particularly useful for recursive execution. When the same service gets executed many times in an execution chain, the operation context is also isolated per service call, and the monitor automatically binds each main operation to the main operation of the immediate higher-level service call.

Recursive operation binding is performed by the Monitor class. By the time a service is called for the second time and requests for the creation of a new operation context, the class identifies the existence of a unfinished operation and automatically binds the new operation as a suboperation of the main operation created in the first call to the service. The monitor manages a stack of non-finished operations and always binds a newly created operation as a suboperation of the operation currently on top of stack. Listing 4.12 shows a monitorable factorial recursive implementation.

Listing 4.12 Example of a monitorable recursive implementation

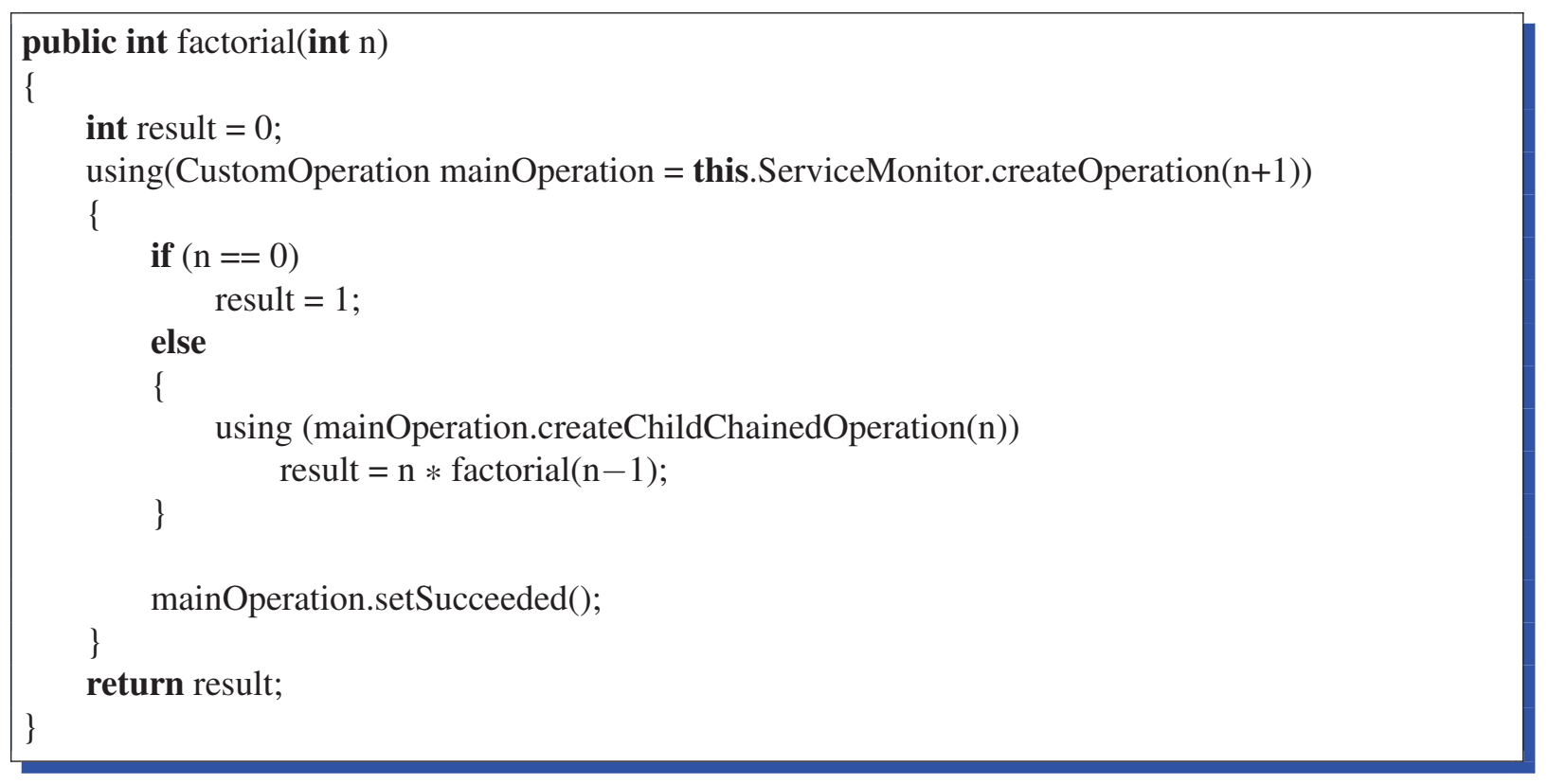

On concurrent execution contexts, such mechanism based on the call stack is not effective, since each different thread has its own independent call stack. In such case, a possible approach is to explictly control the chain of operations and parametrize the recursive method with the parent operation. Therefore, as each service call receives the parent operation as parameter, it can create its main operation as a child of the received parent operation, instead of requesting a new main operation from the monitor.

Another approach for dealing with concurent recursive calls is to isolate each call in a different service provider instance. In such case, each recursive call has its own monitor. In this context, before each recursive call, it is possible to bind the two monitors so that the progress updates of the called service monitor automatically scales to the caller service monitor. 


\subsubsection{Notifications}

Besides automating and encapsulating the process of generating and controlling progress state of operations, the library also provides concrete classes that support the generation of notifications.

While progress state updates introduced in the previous sections provide quantitative information feedback for a service execution, notifications provide qualitative and usually more subjective information feedback about the service execution, by adding messages that describe, in natural language, whatever tasks have been completed, or are still executing, along with alerts and errors that possibly occur during execution. Thus, notifications are a particular kind of real-time logging performed by a service provider.

In Chapter 3, it has been demonstrated two types of notifications: (1) notifications that bind to the execution of an operation and (2) notifications that are arbitrarily generated by the service, with general information about its execution.

For each type of notification, the library defines a concrete class inheriting from the Notification abstract class and implementing specific behavior for the notifications (CustomOperationNotification and GeneralNotification).

Figure 4.4 presents these classes, while the next sections describe them in details.

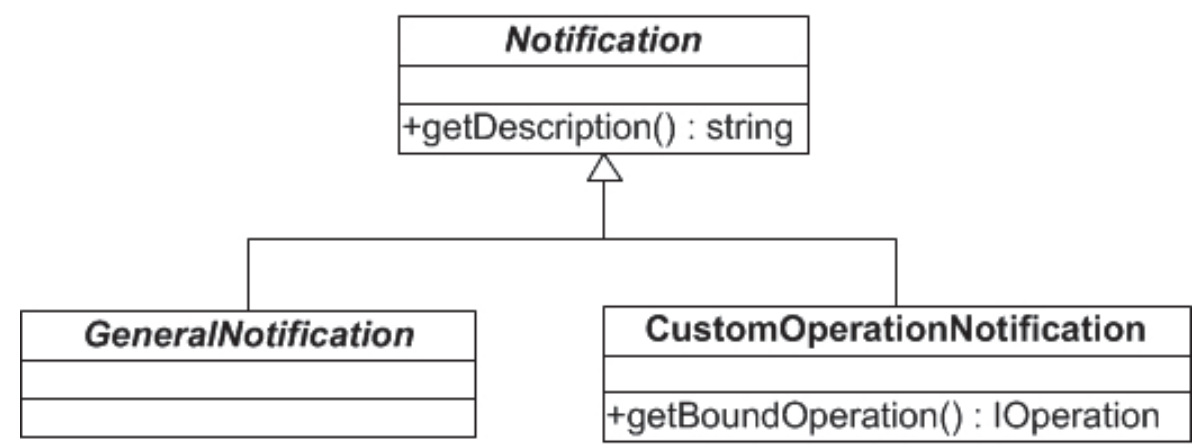

Figure 4.4 CustomOperationNotification and GeneralNotification class diagram.

\subsubsection{CustomOperationNotification}

Every operation has an associated main notification which describes the operation current state in natural language. The main notification is the root of a notification subtree. By the time an operation is created, its associated notification is created and bound to the operation. Any other suboperation created in the context of a running operation will have its notification automatically attached as a child of the parent operation notification, extending its subtree. As can be observed, the notification tree is created automatically as a natural effect of an operation chain.

This type of notification binds to the operation and works as observer for the associated operation. Every update performed by the operation is observed by the notification, which updates itself to reflect the operation current state.

Figure 4.4 shows the class members. Besides the child notifications created automatically by suboperations, it is possible to arbitrarily add new notifications to the notification subtree. The method addNotification allows the service provider to create additional notifications to the tree. 
The following section presents the GeneralNotification class, which represents manually created notifications.

\subsubsection{GeneralNotification}

Besides notifications that bind to operations, the library provides support for general notifications that can be arbitrarily created and attached to an operation-bound notification. These general notifications can be warnings, errors or general information messages that provide businessspecific execution feedback.

The class GeneralNotification represents these general notifications. This class exposes only one single method setStatus that allows determining notification status (errors, alerts, messages, etc.)

The developer can create new instances of GeneralNotification and simply add them to an operation notification, but the library provides an alternative way for creating new notifications automatically bound to the current running operation.

It is possible to create these notifications by making calls to methods writeWarning, writeError or writeMessage, on the ServiceMonitor class, which automatically add new notifications to the main notification of the operation on the top of the operation stack (which represents the currently running operation).

Note that this alternative way of adding notifications is only possible because we assume no concurrent calls creating operations on the monitor. Otherwise, there would exist no operation stack, for there could be more than a single operation concurrently in progress.

Listing 4.13 shows how it works.

Listing 4.13 General notifications creation example

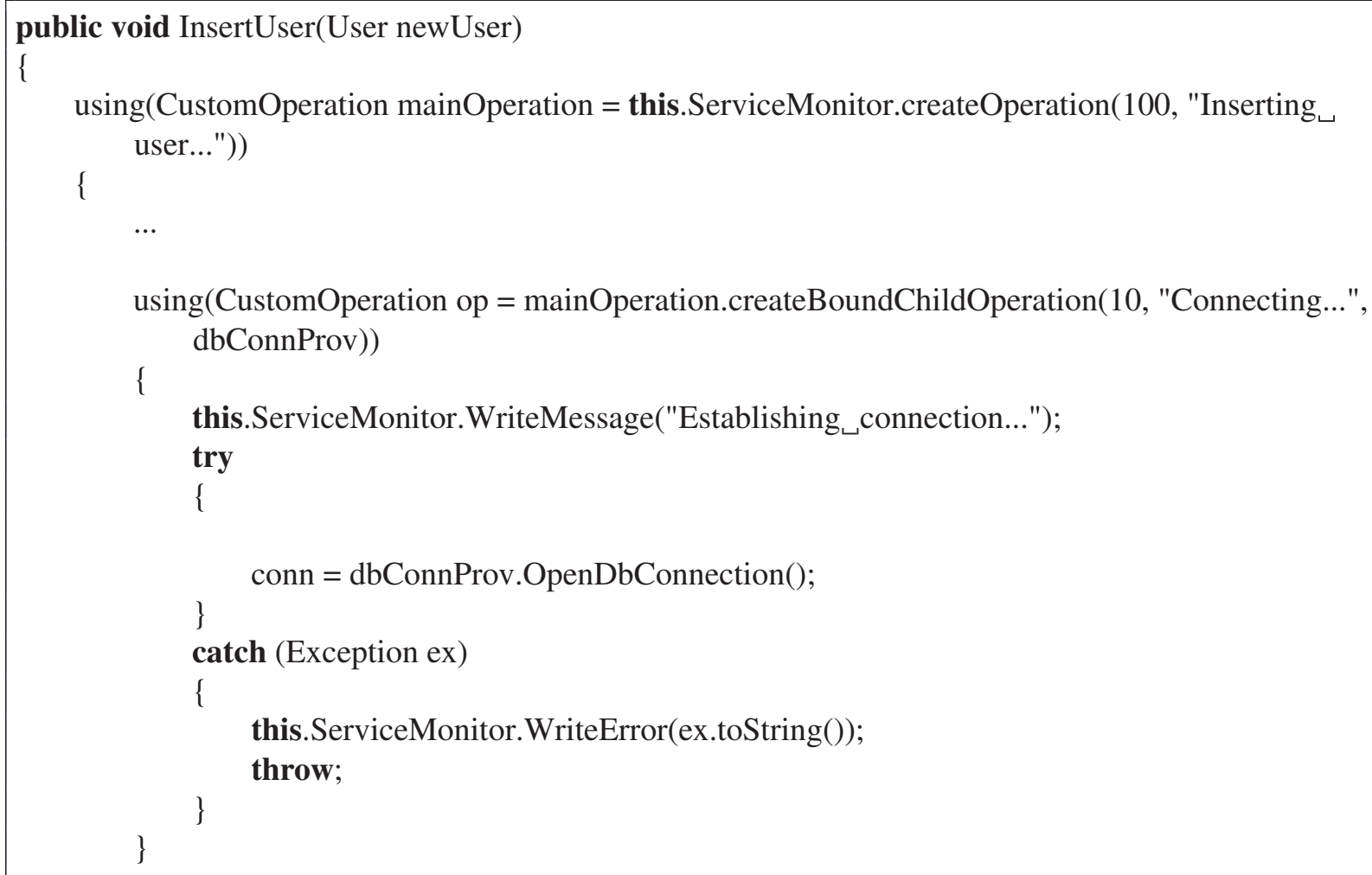




\subsubsection{Service Cancelation}

In order for a service to support cancelation, the service developer needs to add specific code for verifying cancelation requests and perform cancelation action (such as rolling back previous transactions).

The IOperation interface provides a specific state (CancelRequested) that allows the service developer to detect cancelation request.

Cancelation requests are propagated from the main operation to the suboperations still in progress. Thus, higher-level operations propagates cancelation to immediate lower-level operations. Cancelation cascade effect begins on call to Cancel method of IServiceMonitor interface.

A typical cancelation process involves the following steps:

- (1) Service consumer requests cancelation by calling Cancel of ServiceMonitor instance.

- (2) With execution currently in progress, service provider detects cancelation request by observing current operation state change. This is easily checked by invoking the method checkCancelRequested of CustomOperation class.

- (3) Once detected, and assuming the service provider is still in cancelable state, it changes operation state from CancelRequested to Canceling. This state change propagates bottomup to parent operations.

- (4) Service provider performs cancelation according to its business-specific rules.

- (5) Service provider finishes cancelation by calling method setCanceled on the operation and thus changing its state from Canceling to Canceled. State changes automatically propagates to parent operations.

Listing 4.14 shows a typical cancelable service implementation.

Listing 4.14 Typical cancelable service implementation

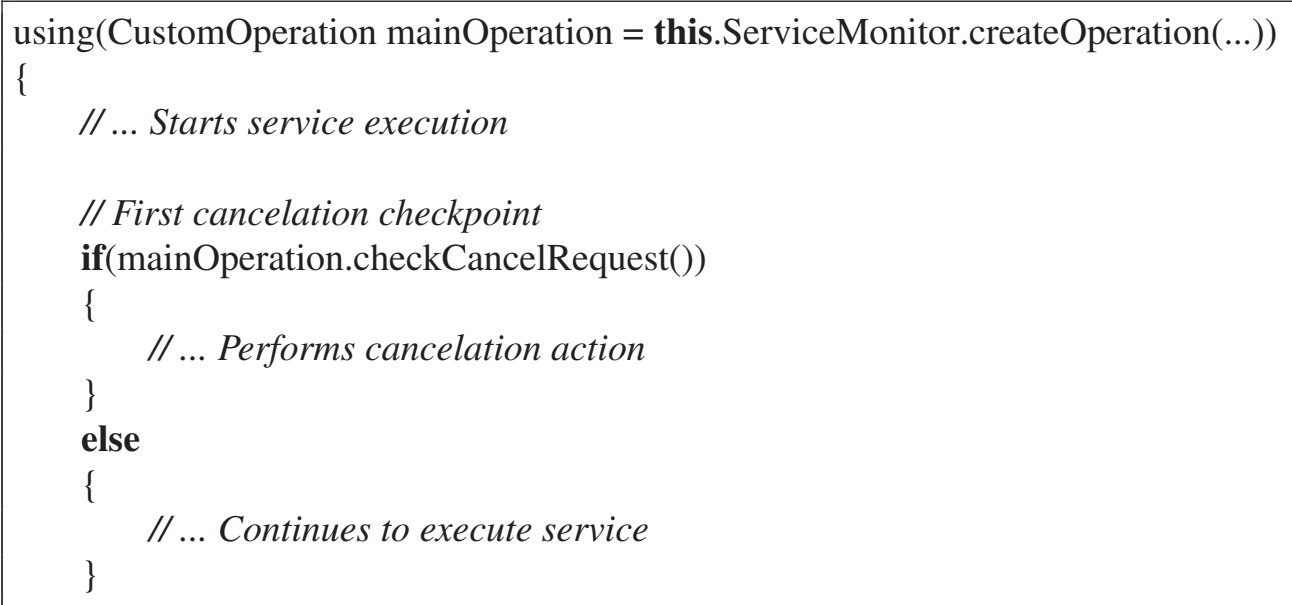




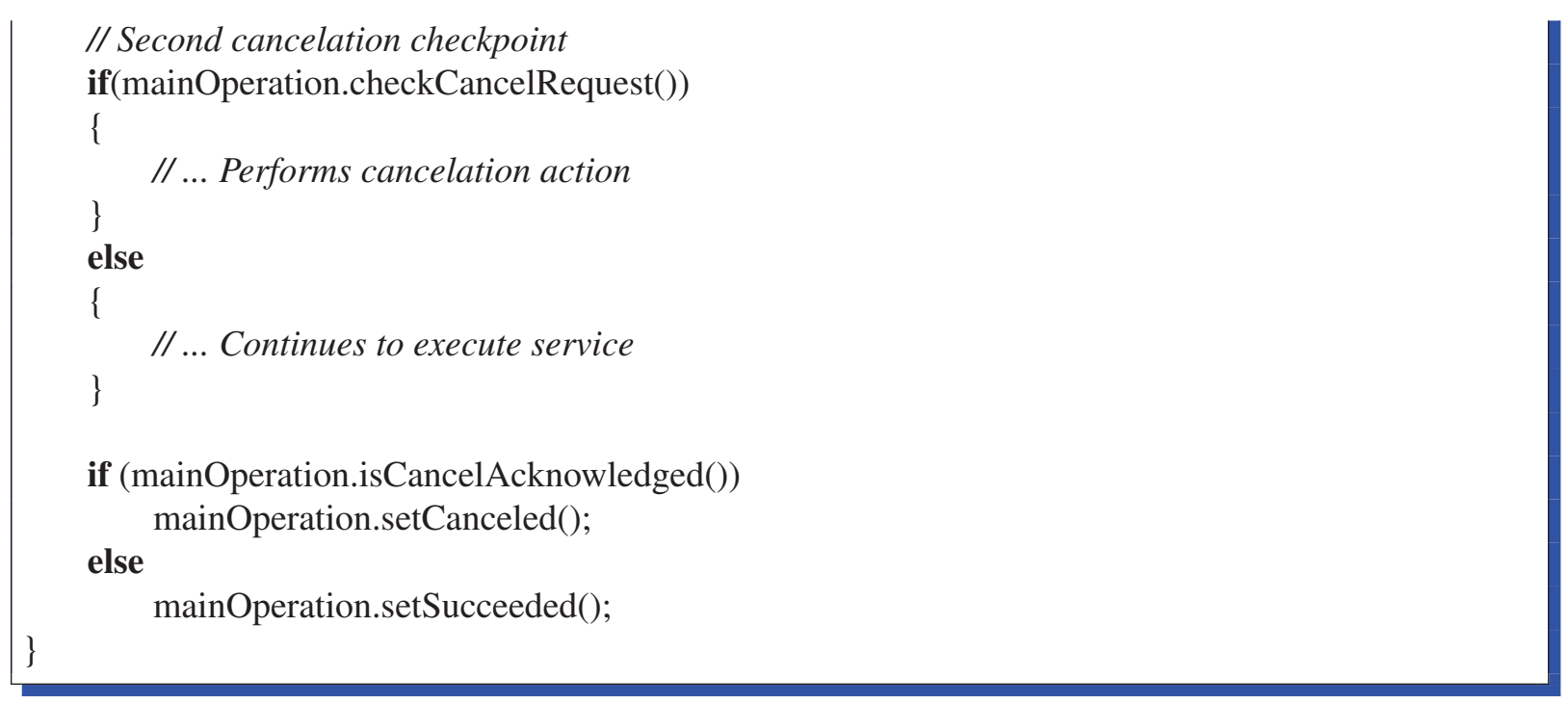

The method isCancelAcknowledged is used to easily verify if a cancelation request has been detected and handled in previous cancelation checkpoints. It suppresses the additional need for the developer to create a local variable in service code to control this. The goal is to add as least code as possible, in order to make it clean and minimize impact on service business-specific code.

\subsection{The Service Consumer Library}

The Service Consumer Library main purpose is to provide graphical components for displaying services monitoring information to the end-user.

These components observe the monitoring information provided by IServicesMonitor interface and displays them to the end-user.

The Service Consumer Library is fully decoupled from the Service Provider Library. One could choose to use any of the libraries without using the other. The Service Consumer Library implementation does not assume that the notifications and progress feedback are provided by using the Service Provider Library components. The implementation is based solely on the framework fundamental definitions.

The following diagram presents the main classes of the library: ProgressViewer and NotificationsViewer.

The next sections describe the classes in more detail.

\subsubsection{Progress Viewer Component}

The ProgressViewer component can be easily added to a user interface of any system and bound to any object implementing IMonitorableServicesProvider. It implements IOperationStateObserver in order to receive updated progress state of currently running operation.

Once added to the application, it displays the currently running operation as a progress bar, showing the percentage of work already completed by the service provider, and displays the current operation description. It also allows the user to request service cancelation.

Figure 4.6 shows how this component looks like in a sample application.

The component exposes the following public methods: 


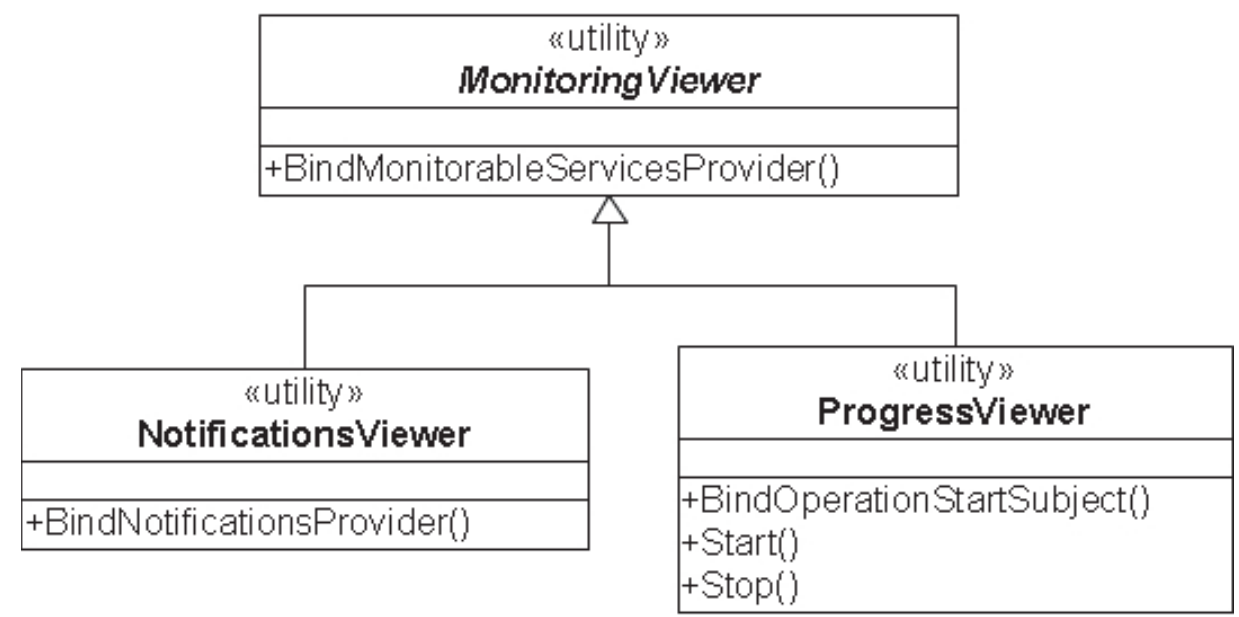

Figure 4.5 Service Consumer Library class diagram.

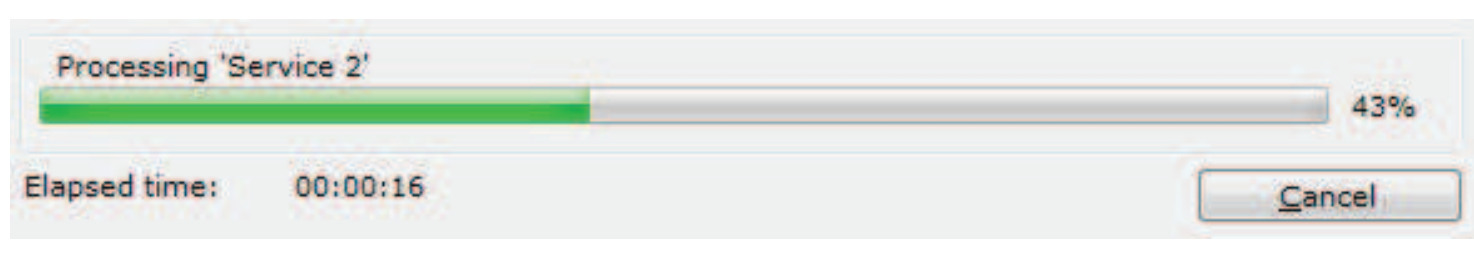

Figure 4.6 ProgressViewer component in a sample application.

- BindMonitorableServicesProvider. This method binds the component to any object providing monitorable services. Then, by the time a service starts execution, the component updates itself to display the main operation state.

- Clear. Restores the component to its initial state, setting the progress bar to zero-percent and no operation is displayed.

\subsubsection{Notifications Viewer Component}

NotificationsViewer component provides a graphical interface for displaying services notifications. It displays a tree of notifications generated by the service provider while running a service. Each tree node represents a notification, and its color and icon changes according to the notification type and state.

Similarly to ProgressViewer, it also binds to any object implementing IMonitorableServiceProvider, but it implements INotificationsProviderObserver, and it updates the graphical notification tree just as new notifications are created. Binding is performed by calling method BindMonitorableServiceProvider exposed by the component.

Figure 4.7 shows how this component looks like in a sample application.

\subsection{Distributed Systems monitoring}

The use of distributed systems in organizations, both in industry and academy, has increased exponentially in recent years, enabled by factors such as ready access to the Internet and the 


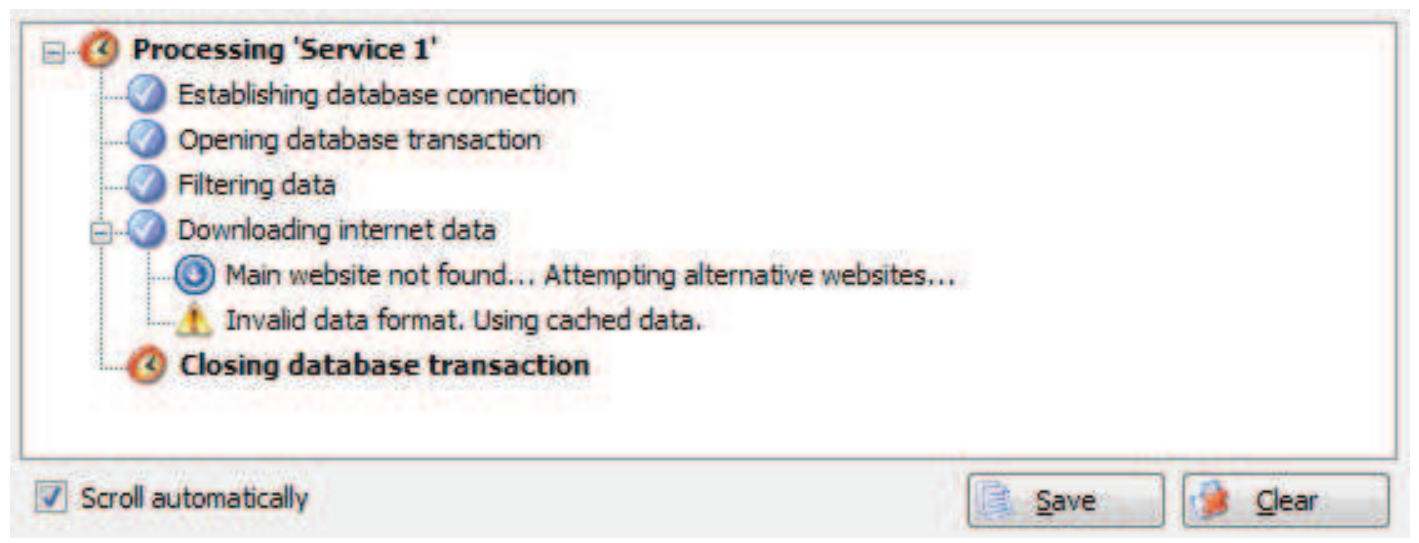

Figure 4.7 NotificationsViewer component in a sample application.

World-Wide Web, the maturity and ubiquity of the HTTP protocol, and the improvement in secure communication technology [ST05]. In the early days, distributed applications communicated using proprietary protocols, and system administrators used ad hoc methods to manage distributed systems. Numerous standards have been developed over the years to ease the costs of deployment and maintenance, with varying degrees of success.

In previous sections, we have covered all monitoring concepts and design that are needed for adding monitoring capabilities to a general object-oriented application, but we intentionally did not provide any special kind of implementation consideration regarding monitoring in a distributed system environment. In this section, we review some fundamental concepts of service-oriented architectures (SOA), since it is meaningly present in both industrial and academic environments, discuss some specific considerations for adding execution monitoring in such distributed scenarios, and finally provide a brief discussion regarding the development of monitorable distributed services.

\subsubsection{Service-Oriented Architecture (SOA)}

Service-Oriented Computing (SOC) and Service-Oriented Architecture (SOA) are a vast topic. In fact, there are entire books covering this topic of the Distributed Systems discipline, and it is not our intent to provide detailed information on the technology. Instead, we provide a very brief overview on the subject before we start discussing the monitoring problem within a SOA context.

Businesses need to connect processes, people, and information both within the organization and across organizational boundaries to subsidiaries or trading partners. A lack of integration among IT assets (systems, applications and data) makes it difficult for IT to respond quickly and effectively to changing business needs. This inflexibility increases costs, decreases customer responsiveness, hinders compliance, and decreases worker productivity. In short, a lack of integration is the biggest challenge that organizations face in their efforts to remain competitive and grow [Cor06].

Service-Oriented Computing (SOC) utilizes services as the constructs to support the development of rapid, low-cost and easy composition of distributed applications. Services are autonomous, platform-independent computational entities that can be used in a platform independent way. Services can be described, published, discovered, and dynamically assembled for 
developing massively distributed, interoperable, evolvable systems. Services perform functions that can range from answering simple requests to executing sophisticated business processes requiring peer-to-peer relationships between possibly multiple layers of service consumers and providers. Any piece of code and any application component deployed on a system can be reused and transformed into a network-available service. Services reflect a "service-oriented" approach to programming, based on the idea of composing applications by discovering and invoking network-available services rather than building new applications or by invoking available applications to accomplish some task. Services are most often built in a way that is independent of the context in which they are used. This means that the service provider and the consumers are loosely coupled [MPL08].

Key to the SOC concept is Service-Oriented Architecture (SOA). SOA is a logical way of designing a software system to provide services to either end-user applications or to other services distributed in a network, via published and discoverable interfaces. A well-constructed, standards-based Service Oriented Architecture can empower a business environment with a flexible infrastructure and processing environment. SOA achieves this by provisioning independent, reusable automated business process and systems functions as services and providing a robust and secure foundation for leveraging these services [MPL08].

Thus, SOA is a design approach that allows organizing existing IT assets such that the heterogeneous array of distributed, complex systems and applications can be transformed into a network of integrated, simplified and highly flexible resources [Cor06].

SOA provides the design framework to integrate siloed applications so that their functionality can be accessed as services on a network. Most commonly implemented through standardsbased, technology-neutral Web Services, SOA breaks down monolithic applications into a suite of services, implementing functionality in a modular fashion [Lin07].

Ideal services are designed to be stand-alone, autonomous, and loosely coupled. Thus, they can be readily combined and recombined into composite applications according to the changing needs of the organization [Cor06].

According to David Sprott and Lawrence Wilkes, it seems probable that eventually most software capabilities will be delivered and consumed as services [DS04]. They may still be implemented as tightly coupled systems, but the point of usage (to the portal, to the device, to another endpoint, and so on) will use a service-based interface. Despite how reasonable and reliable is such a prediction, Service-Oriented Architectures are already the major adopted strategy for implementing distribution and integration [MPL07].

In this context, we should pay a special attention to SOA specific characteristics, guidelines and architectural recommendations, in order to ensure full compliance and compatibility of our framework and library in a general, supposedly standardized, SOA scenario.

With respect to monitoring, distributed services introduce two major concerns:

- Concurrent execution. Since distribution implies concurrence, we need to deal with the execution of concurrent operations when considering the execution of a distributed service. In practice, however, most development platforms and tools provide proxy class generators that hide the communication details and expose the distributed services as ordinary local methods that can be called synchronously. In any case, if the distributed service execution is actually asynchronous, it is possible to implement the monitoring operation by using the code pattern explained in Listing 4.5. Therefore, as the concurrence 
concern has been addressed already, and there are no additional difficulties because of distribution, this concern is not the focus of this section.

- Distributed callback events. The mechanism behind providing progress feedback and state updates is based upon callback interfaces (implemented using the Observer Pattern). However, such callback interfaces are based upon local object references which are not present in the context of a distributed service execution. This is the primary concern for monitoring distributed services and therefore it is the focus of the discussion in this section.

\subsubsection{Events, Decoupling and SOA}

Many SOA implementations are based upon the request/response interaction pattern, where a service requestor identifies a service that it wishes to use and then sends it a request message. A second entity, the service provider, accepts the request message, processes it, and then sends a response message. This is a pattern that is familiar to any programmer who has made a procedure or function call in a procedural programming language or who has invoked a method in an object-oriented language or distributed object system. Indeed this pattern is so familiar that programming interfaces and tools (for example, those used with Web services) often hide the underlying message exchange; these tools present a programming model that looks like a simple procedure call [PN05].

Event-based programming has been around for many years and has been applied in many areas, such as user-interface systems designed upon the MVC architectural pattern [Ree79]. Event-based programming features an entity that represents an occurrence (something that has happened). In object-oriented systems this is usually termed an event object; in messageoriented systems it is variously referred to as a message, event, or event message. In contrast to the request/response pattern where the request and response messages are frequently hidden from the programmer, in event-based programming the event (be it a message or object) assumes center stage. Applications explicitly produce and consume events, and the producing application has a relationship with the event that it produces, rather than a direct relationship with the applications that consume the event, stimulating consumer/provider decoupling. A consumer of events indicates (through a registration process) the events in which it is interested, and it interacts with the event itself, rather than with the application that produced the event. The term notification pattern is often used to refer to the interaction pattern that involves registration of consumers and subsequent dissemination of events [PN05].

Just as with Service Oriented Architecture, our framework also stimulates decoupling between consumers and providers. All monitoring information is produced within a service provider without making assumptions on the nature of the service consumer. The entity receiving monitoring information, on the other hand, also does not make assumptions on the service provider nature. All the monitoring information is provided on an event-based interaction. In order to enforce decoupling while supporting the event subscription and notification process, we took advantage of the Observer Pattern, as explained in Section 3.2.1, and implemented by INotificationsProviderSubject (3.2.2.1) and IOperationStartSubject (3.2.4).

The Observer Pattern is very easily implemented on any object-oriented system, because we can easily hold references/pointers to the objects involved. Thus, we can think of notification as a simple procedure call on an object reference that we held upon the subscription process. For 
example, one entity interested in receiving a notification will simply implement the Observer interface and pass its own reference to the Subject interface, by calling the attach method. The Subject will simply hold this static reference, and will simply call the notify method by the time an event occurs.

On message-oriented systems — such as SOA implementations based on Web Services and on major distributed systems - , we often do not have object references representing service consumers and providers. Notice that although such distributed system characteristics add an additional concern for the implementation of monitorable services, they do not require any change on our framework model, which is still compatible with a general consumer/provider object-oriented relationship, whether they are implemented as distributed services of a SOA architecture or not.

In practice, in order to execute a distributed service, a connection to an Enterprise Service Bus (ESB) must be established through an available endpoint, and request/response messages might be exchanged for the desired service to be effectively executed. This communication process is often performed by a proxy component that handles the communication details. In this scenario, in order for a service to become monitorable, we would need to add monitoring message exchanges between provider and consumer, while the service is executing. The SOA implementation would need mechanisms for the service provider to send additional notifications about the service execution while it is sill executing, and for the service consumer to receive the messages and notify the original consumer by calling notify on the Observer of the objectoriented model.

To better illustrate, suppose we are implementing a monitorable service for a financial transfer between two bank accounts. The method transfer needs to execute a distributed service as a suboperation, say credit distributed service. Figure 4.8 shows the sequence diagram for this scenario.

The framework establishes the communication protocol between the Consumer and the Service Provider, which is determined by the IMonitorableServicesProvider interface, and does not provide any concrete implementation, as explained in Chapter 3, and is based on the $\mathrm{Ob}$ server Pattern. The Service Provider Library, on the other hand, despite providing a concrete implementation, does not impose technology-specific limitations. Thus, we assume, whatever the technology involved, the Service Provider will be able to notify the consumer based on the Observer principle.

The problem arises when the Service Provider needs to start a long suboperation on a distributed layer and needs to provide detailed progress feedback upon the suboperation execution. This process is represented by the interaction between the Service Provider and the Proxy. To achieve this goal, the Proxy would need to notify detailed information on the distributed operation execution. For the Proxy to notify progress monitoring information, the ESB needs to support this sort of notification.

In recent years, various analysts and software vendors have started to use the term Eventdriven architecture (EDA) to describe software architectures that utilize event-based programming [Mic06]. Some SOA implementations are designed in conformity with the EDA concept. When the SOA implementation conforms to EDA, it is easy to implement the monitoring notifications described between the Proxy and the ESB. When SOA implementations are based on pure request/response implementations, on the other hand, it will be harder to implement such notifications. 


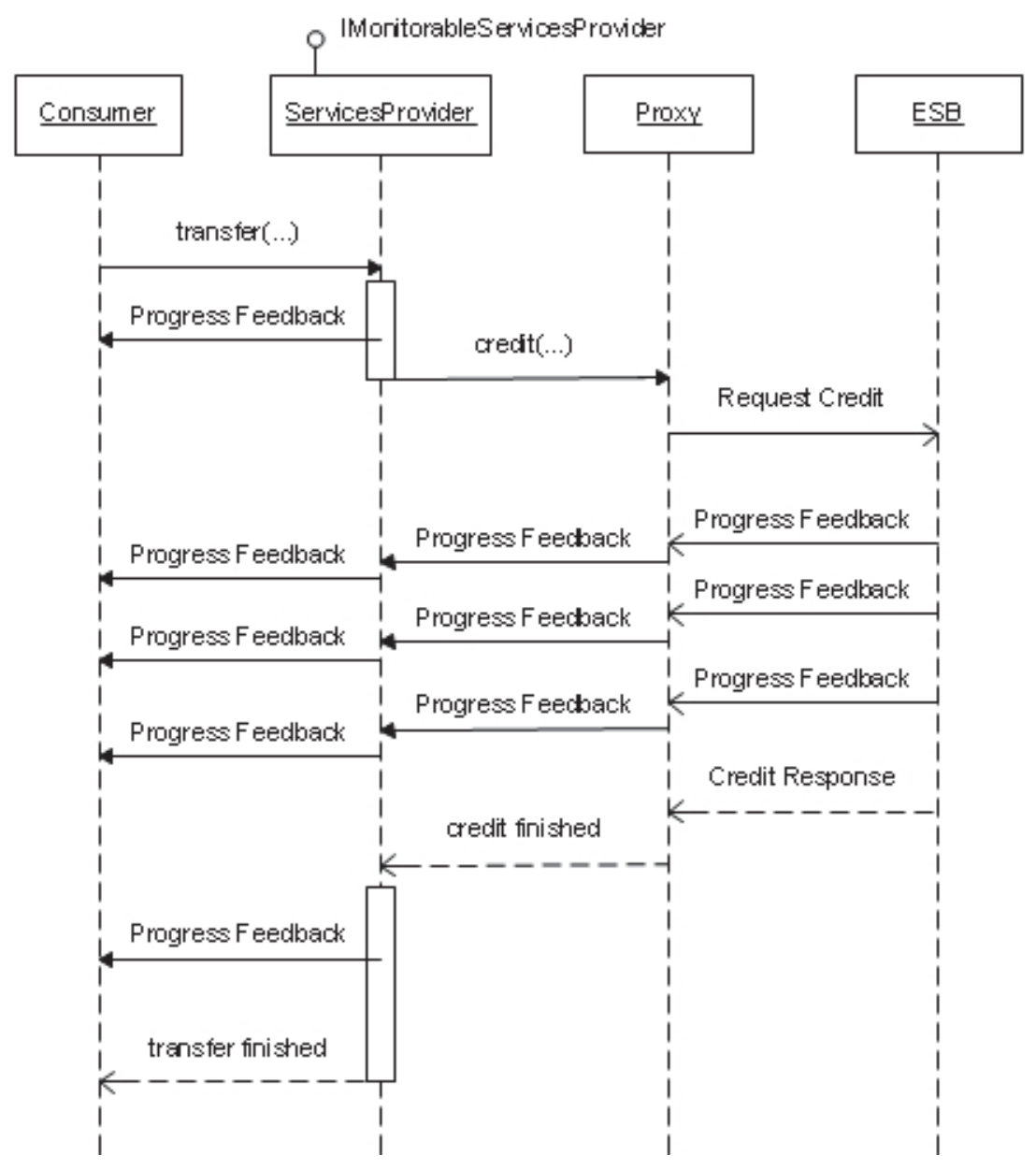

Figure 4.8 Monitorable distributed service sequence diagram.

Today, there already exist standardized technologies and mature specifications for supporting Event-driven SOA implementations. One example, for Web Services based implementations, is The OASIS Web Services Notification (WSN) family of specifications, which defines a standard interoperable protocol through which Web Services can disseminate events. The intent of WSN is to define a set of royalty-free, related, interoperable, and modular specifications that allow the notification pattern to be modeled in an explicit and standardized fashion. The benefits of such standardization include interoperation between application entities written by different authors, as well as interoperation between different publish/subscribe messaging middleware providers [PN05].

As it would be trivial for us to deal with event-driven SOA implementations, for they already implement mechanisms for easily handling events subscription/notification, let us focus on the most difficult scenario, when the distributed architecture does not provide native support for handling events.

Even on a SOA implementation purely based upon the request/response pattern approach, it would still be possible for the proxy to notify monitoring events, although it would not be the most appropriate method and definitely not the most optimal for network traffic. For us to emulate events in this scenario, we will need the service implementation to maintain a queue of undelivered monitoring events, and the Proxy would need to regularly consume the queue 
while the service executes, in parallel, by invoking an specific service on the ESB for consuming undelivered monitoring events. When the Proxy consumes the queue, it can emulate the corresponding events in such a way that the Service Provider will not even notice the difference.

In this case, in order to encapsulate the complexity, we would suggest another extension of the framework by creating a library that does the job of buffering events on both consumer and provider side, and dealing with the details of the proxy-ESB interaction. It is out of the scope of this dissertation to detail this implementation, but still we suggest an idea for gathering monitoring information during the proxy-ESB interaction: for every monitorable service invocation performed by the proxy on the ESB, the proxy would need to immediately start an asynchronous loop for collecting monitoring information, during the service execution.

\subsection{General Purpose Versus Domain-specific implementations}

In previous sections, we have provided a general solution for implementing monitorable services on object-oriented systems, and discussed how to extend this support on a distributed scenario. We have presented a framework model and libraries that serve general purpose system development. This approach has the obvious and inherent benefits of being general: whatever the system being built, if it is based on standard object-oriented paradigm, it is possible to implement monitoring capabilities with little effort by reusing this framework model. Conversely, this approach leads to a perhaps not so obvious disadvantage: general purpose solutions are often not optimal (the better possible solution) for specific scenarios.

The monitoring framework and libraries have been designed with general purpose intent, with the goal of minimizing technical assumptions upon the platform, business nature, technological architecture or infrastructure of the application under consideration. For example, if we had focused on workflow-based services, we would have designed a simpler framework and library, easier to use and utilizing workflow-specific characteristics.

We could have decided to gather some distinct specific scenarios and then designed corresponding distinct monitoring frameworks and libraries, optimized for each one of them. There might exist not so much specific scenarios for us to deal with, and the required effort, although certainly greater, would still make the project viable. Nonetheless, there is still a very strong reason for us to decide for a general purpose framework and library: in spite of being general purpose, the design is absolutely extensible and reusable, allowing one to create domain-specific framework as an extension from the general purpose framework, reusing all fundamental concepts and basic algorithms, with no prejudice or limitation for making it optimal in the given specific scenario.

Thus, we view our general purpose framework and library not as a final end-user product - although it is fully ready to use for general-purpose scenarios, as demonstrated in the case study performed (see Chapter 6) — but as a base design, a trunk for domain-specific framework branches.

Our design enables direct usage of the framework and library to add monitoring capabilities, considering an empty set of assumptions, but it also serve as a basis for a domain-specific framework customization to improve monitoring usage. By the time we add assumptions and limit the framework usage context, one may be able to directly improve or eliminate some inconveniences of monitorable code.

The top two most inconvenient characteristics of the monitorable code shown in previous 
sections are Code Tangling and Code Scattering, described as follows:

- Code Scattering. When similar code appears throughout many components and modules of an application. It often happens with crosscutting concerns, which often results from non-functional requirements - in fact, this the case of monitoring concern. We have seen that every monitorable service has to initialize a monitor, create the main operation, create the suboperations, manually signal operations successful completion, and check for cancelation requests. As a result, the code patterns involved in these actions are replicated and distributed throughout the application modules.

- Code Tangling. When two or more concerns are implemented within the same component or module of an application. It also happens with the execution monitoring concern, for its tight coupling nature with business-specific implementation. This leads to "spaghetti code" [Kic99] that is difficult to understand and maintain.

Such inconveniences can be completely eliminated if we waive on the general purpose nature of the framework by adding assumptions upon the architecture, design and form of the service implementations. One example is that if we are able to assume that every monitorable service will be implemented in a workflow-based design. The next section describes how the framework could be extended to optimally deal with such a specific scenario.

\subsection{Workflow-based Extension}

In Section 2.5, we provided an overview of Workflow concepts, its powerful capability for separating business logic from its actual implementation, and how we can make use of these characteristics in the context of service execution monitoring.

Just as almost everything in the vast area of Software Development and Engineering, one interested in developing a service as a workflow will often implement it on top of a base Workflow Framework; regardless whether it is a commercial, academic or open framework, it seems improbable that one would implement the workflow fundamental constructs from scratch. A decision on the contrary, besides the waste of unnecessary effort, would be a rough denial of software reuse best practices and recommendations, and would lead to deviation of focus from the business actual requirements to workflow-specific implementation.

A Workflow Framework often defines abstract class definitions for representing activities, their transitions, processes and a built-in engine for managing their execution. Additionally, they provide a set of useful workflow constructs for representing execution flow details, such as conditionals, parallelism, scheduling, signals, loops, etc. The most complete frameworks also provide native constructs for creating transactions, defining cancelation handling, fault handling and recovering, etc.

This section discusses how the framework and library can be extended in order to improve its usage on the context of business implementations based on workflows. For us to provide a final, ready-to-use design definition, we could have chosen an existing Workflow Framework for us to use as basis for our definition, such as the Microsoft Windows Workflow Foundation (WWF) [Cha10], which is built on top of the Microsoft .Net Framework [Net11], a very mature, complete, license-free and widely used framework for building workflow-based implementations, developed by Microsoft Corporation. 
However, instead of choosing a specific workflow framework and showing how it would be extended to support monitoring, our purpose in this section is to provide a general description on how any Workflow Framework could be extended. For this reason, we considered more appropriate to provide an abstract description of typical workflow classes, as representing part of a fictitious workflow framework, and provide examples on how to make them monitorable.

\subsubsection{Workflow Activity Specification}

Activities are the building blocks of workflows. Every workflow is a composition of one or more different activities that should be executed in a proper order and according to a specific criteria. Thus, for a business workflow to be monitorable, its composing activities must be individually monitorable.

Before we actually describe the design of a monitorable workflow activity, we need to define the base class for representing a Workflow Activity on a general non-monitorable Workflow Framework. Figure 4.9 presents such Activity class.

\begin{tabular}{|l|}
\hline \multicolumn{1}{|c|}{ Activity } \\
\hline -parent : Activity \\
-childActivities : List<Activity> \\
\hline +execute() \\
\hline
\end{tabular}

Figure 4.9 A general representation for a general non-monitorable Workflow Activity.

A typical Activity in a general workflow framework provides attributes that enable traversing the workflow both top-down and bottom-up. In the example, these are represented by the attributes parent and childActivities. In addition, the Activity class also provides an abstract method which is called by the Workflow Engine in order to fire the activity execution. In the example, this is represented by the execute method. It is important to understand that the execute method is not originally related to monitoring. It is solely related to the normal workflow execution and is originally intended for the activity business-specific functional implementation. Therefore, it is defined in a general non-monitorable Activity class.

Our strategy to simplify the monitoring model in workflows relies upon the very simple idea of creating a one-to-one mapping between workflow activities and our definition of monitorable operation. Thus, every monitorable activity should associate to exactly one operation of our monitoring framework. The concept of suboperation arises naturally by the time we compose an activity as a new workflow of subactivities. Thus, the outermost main activity, which does not have a parent activity, associates to a main operation, while its subactivity operations are child operations of the main operation. The fact that an activity may or may not have a parent activity will be used to determine whether a monitorable activity should scale its progress events in order to update the parent activity progress.

For an activity to be monitorable, we consider the following aspects:

- Before a monitorable activity starts execution, it must traverse all of its composing activities in order to calculate the total activity cost. This operation is easily implemented with a recursive in-depth navigation along the child activities. After calculating the total activity cost, it could provide a first execution progress feedback with zero-percent but not idle progress state. 
- After a monitorable activity ends execution, it must provide a final progress state feedback, stating whether the activity completed successfully or not.

- When a child activity provides progress state feedback, it must scale costs and update the overall activity progress state.

- When a child activity ends execution, it must reevaluate cancelation state in order to decide normal flow execution or start a cancelation (rollback) flow.

These simple aspects lead to a regular strategy for implementing monitorable workflows and would guide a workflow-based framework extension.

Therefore, in order for us to add monitoring capabilities to such a general activity, we would need to define a MonitorableActivity class supporting each one of the aspects listed above. Figure 4.10 presents the MonitorableActivity class diagram.

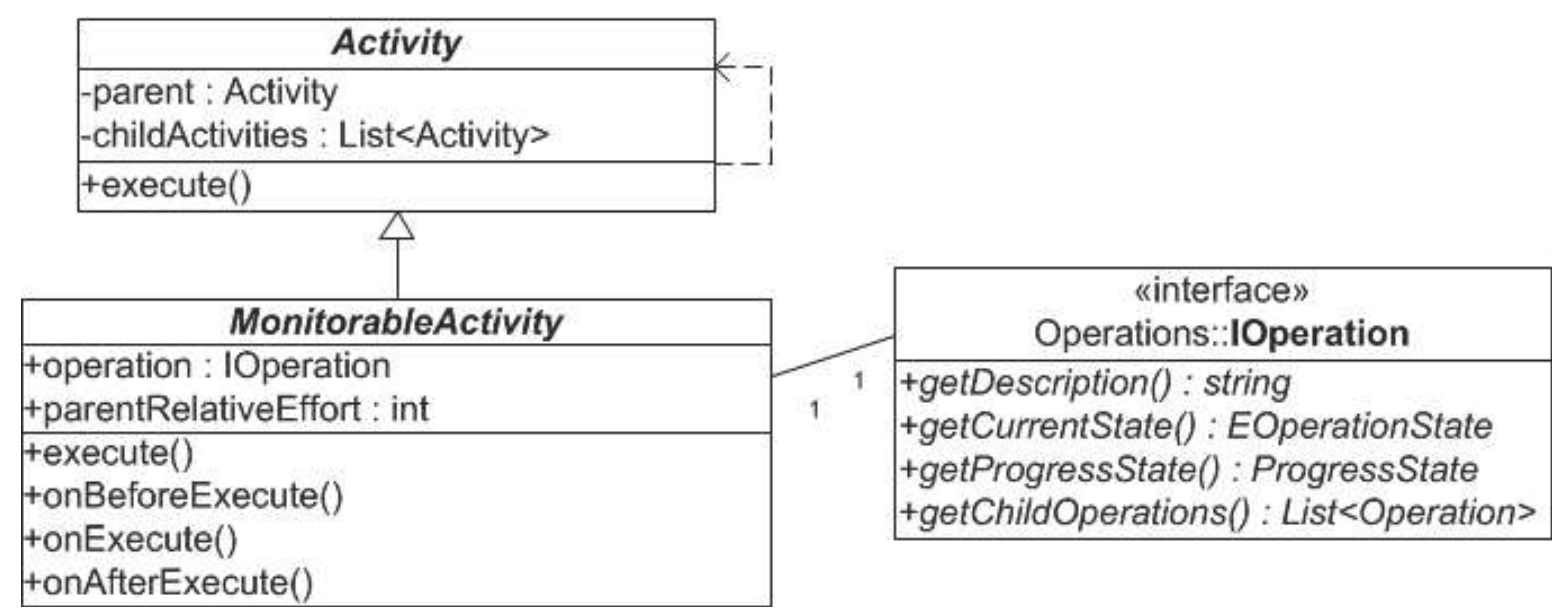

Figure 4.10 MonitorableActivity Class Diagram.

The idea of the Workflow-based solution presented in this section is very similar to the one of the Aspect-oriented approach discussed in the Section 2.5, since it relies on splitting the monitorable method execution into strategic flow control points, so that it has implicit and modularizable code for before and after each operation executes. These points allow the introduction of reusable monitoring logic, such as creating operations, binding it to parent operations and providing progress feedback. Besides the structural differences when comparing to the Aspect-oriented approach, one important difference in this solution is that it forces every monitorable service to be implemented by defining activity classes inheriting from MonitorableActivity class. By doing so, it imposes a specific way of implementing the monitorable services, but it also creates an isolated place for encapsulating the monitoring logic, storing costs and accessing business-specific context.

The execute abstract method defined in the Activity base class would be implemented in the MonitorableActivity class and explicitly divide the execution into three abstract parts: onBeforeExecute, onExecute and onAfterExecute. The explicit division of the execute method allows implementing base monitoring code that should execute before the activity actually executes and immediately after it executes. While onBeforeExecute and onAfterExecute are defined as 
concrete methods, with a default implementation, the onExecute method is defined as abstract and should perform business-specific code on a concrete monitorable activity implementation.

The attribute parentRelativeEffort is used to represent the amount of work that the activity represents relative to the context of the parent activity execution. Its value is not inherent to the activity itself and should be set by the parent activity before it starts execution. For example, in a very large and complex activity workflow, an activity $\mathrm{X}$ may represent $5 \%$ of the total amount, while in a very simple activity workflow, the same activity $\mathrm{X}$ may represent $80 \%$ of total work.

The attribute operation is used to bind the activity to its corresponding IOperation on the monitoring framework. The IOperation creation would be performed automatically before the activity starts execution. If the workflow framework provides any native support for activity description, then the same description would be automatically used for the operation.

It is out of the scope of this dissertation to present every single detail of such an implementation. Our intention is to demonstrate how the same framework could be extended and reused for more specific scenarios, such as in workflow-based implementation. Therefore, we focus on presenting the design approach and demonstrate how the base monitoring framework can be extended to create a more optimal solution for a specific scenario.

Such monitorable workflow-based design would allow a complete modularization of the monitoring concern within the MonitorableActivity class, reducing code tangling and scattering to zero and therefore eliminating monitoring code quality problem. For example, one could easily redesign the InsertUser service shown in Listing 4.4 as a workflow just as shown in Figure 4.11.

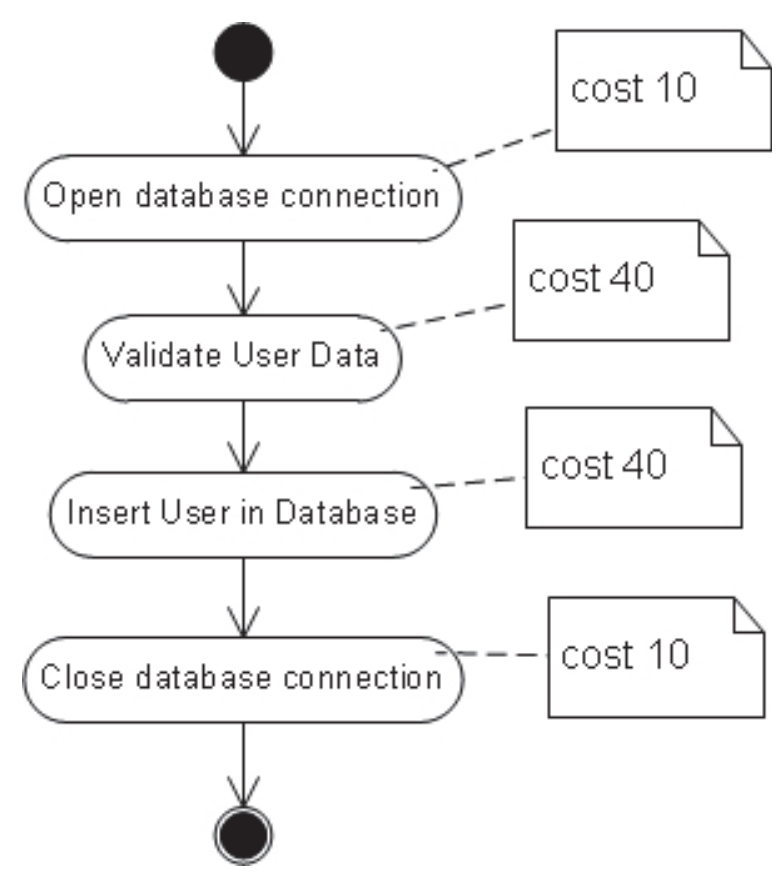

Figure 4.11 Workflow for "Insert User" service.

In order to implement this workflow, all we need to do is to implement one class for each workflow activity. Then, the implementation should look like that in Listing 4.15.

Listing 4.15 User insertion monitorable workflow 


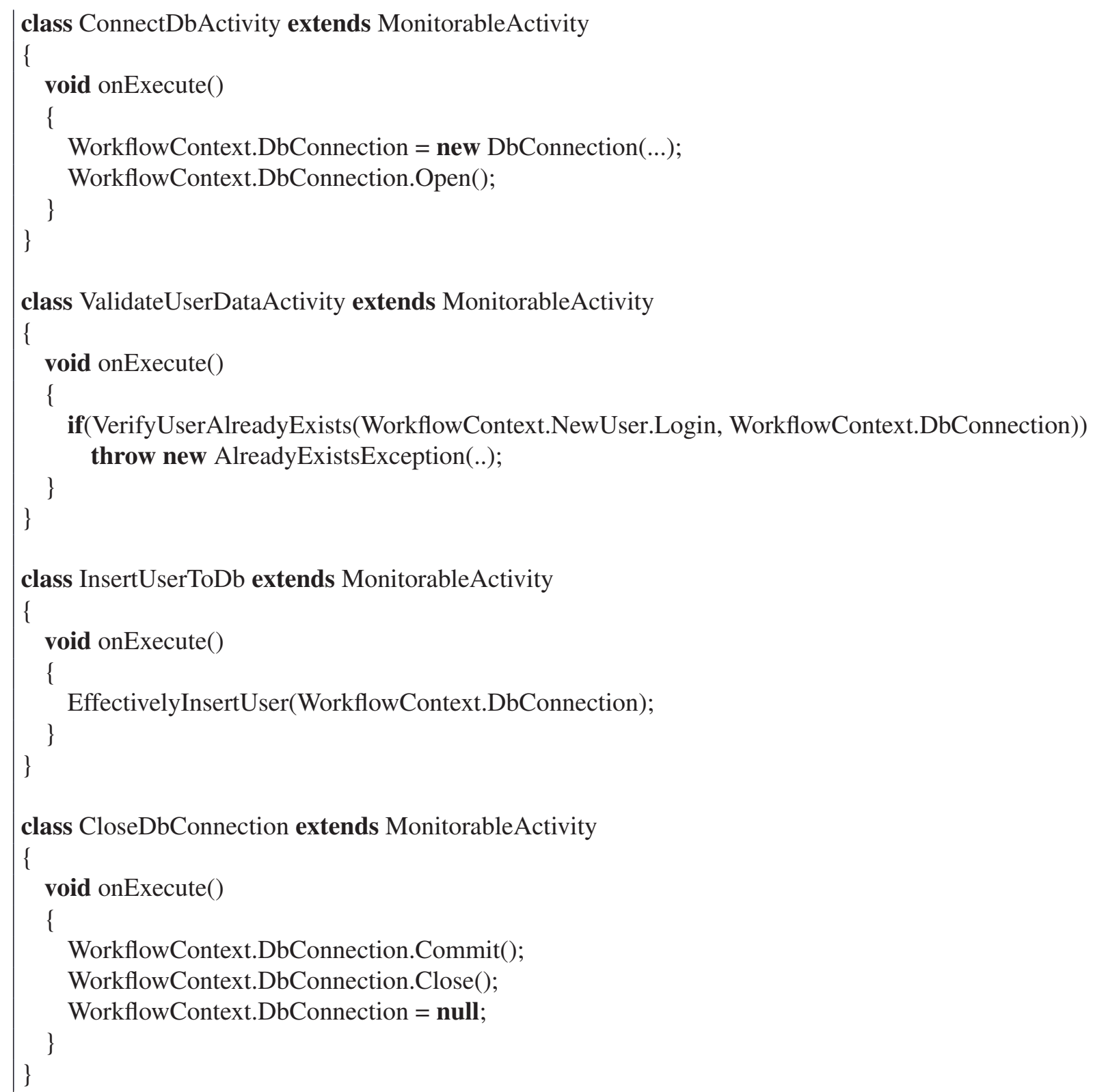

The order in which the activities execute should be coordinated behind the scenes by the workflow engine. We would still need to define an activity in the Workflow Framework for representing the main InserUserActivity, which would be a composition of the sequence of activities defined above, with their corresponding execution costs.

Notice that the code became much more cleaner with respect to monitoring (there is no apparent monitoring code at all) by completely eliminating monitoring code from business code, when comparing to the non-workflow implementation provided in Listing 4.4.

One could hastily conclude that a workflow-based approach is always the best alternative for implementing monitorable services. This statement can be true for simple services that are easily defined as workflows, but it is definitely not true for every situation, due to the following reasons.

- It forces service implementation to split up. Some services have complex implementations, involving algorithms that are not easily split and modularized into different classes, 
since they may share a large context of local variables and temporary objects that make the business code implementation much more complex if we force them to split up into different method implementations.

- It is not flexible for dividing progress state of atomic workflow activities. When an activity is a composition of other activities and the costs are distributed uniformly along them, progress feedback is uniform because the state of a main activity responds to the change of state in the child activities. On the other hand, if we had a single atomic activity consuming a major part of the overall workflow cost, then we could have abrupt progress state changes, such as from $0 \%$ to $80 \%$. To solve this problem, we would need to allow for an atomic workflow activity implementation providing partial progress state feedback, and it would lead again to local tangling of monitoring and business code within the activity class implementation.

\subsection{Related Work comparison}

Once we have presented the framework and libraries for monitorable services implementation, let us outline a comparison of this solution and the approaches discussed in Chapter 2. Table 4.2 summarizes our first impressions.

Table 4.2 Approach comparison.

\begin{tabular}{|c|c|c|c|c|c|c|}
\cline { 2 - 6 } \multicolumn{1}{c|}{} & Ad-hoc & Eclipse & AOP & $\begin{array}{c}\text { AOP with } \\
\text { Metadata }\end{array}$ & Workflow & $\begin{array}{c}\text { Proposed } \\
\text { solution }\end{array}$ \\
\hline Generality & Good & Good & Good & Good & Bad & Good \\
\hline Constrainability & Bad & Medium & Good & Bad & Bad & Good \\
\hline Decoupling & Bad & Good & Good & Good & Good & Good \\
\hline Tangling & Bad & Bad & Medium & Good & Good & Medium \\
\hline Scattering & Bad & Bad & Medium & Medium & Good & Bad \\
\hline Code legibility & Bad & Medium & Good & Good & Good & Medium \\
\hline Code regularity & Bad & Good & Medium & Medium & Good & Good \\
\hline Industry adoption & Good & Good & Bad & Bad & Good & Good \\
\hline Platform-independent & Good & Good & Medium & Medium & Good & Good \\
\hline Distribution & Bad & Medium & Good & Good & Medium & Good \\
\hline A posterioridevelop. & Bad & Good & Medium & Bad & Bad & Good \\
\hline
\end{tabular}

We consider several properties for comparing the different approaches:

- Generality. We consider whether it is a general purpose approach, applicable to general development scenarios, or whether it is based upon domain-specific scenarios. For example, we classify the Workflow-based design as specific, since it is only applicable to workflow-based business implementations. 
- Constrainability. We consider whether the approach adds constraints to the coding style. Form example, we classify Aspect-oriented approach with Metadata (method annotations) as bad regarding this property, since it imposes a specific way spliting up method implementation.

- Decoupling. We consider whether the approach allows for the decoupling of service consumers and providers, providing support for layered architectures. For example, we classify the Ad-hoc approach as bad regarding decoupling, since it tends to generate tightly coupled code.

- Tangling. We consider whether the approach allows reducing code tangling. For example, we classify both the Ad-hoc and Eclipse approach as bad since they tend to generate a high rate of code tangling.

- Scattering. We consider whether the approach allows reducing code scattering. For example, we classify the Eclipse approach as bad since it does not modularize subtasks management and percentage aggregation logic, which leads to scattered code along distinct code modules.

- Code legibility. We consider whether the resulting business code legibility is affected by the monitoring implementation. For example, we classify the Ad-hoc approach as bad since it adds unnecessary complexity to the business code.

- Code regularity. We consider wheter the approach enables a regular coding style for monitoring code implementation. For example, we classify the Aspect-oriented approaches as medium, since they do not offer a regular coding style (sometimes it uses cflow-based pointcuts, sometimes it uses aspect inheritance, sometimes the annotations alone are sufficient).

- Subtasking. We consider whether the approach enables automatic generation and management of subtasks, inferred from the call stack, without the need for additional code to explictly handle subtasking.

- Industry adoption. We consider whether the approach relies on programming paradigms and techiniques widely adoped in industry practice. For example, we classify the Aspectoriented approaches as bad since they are very far from pure object-oriented paradigm regarding industry adoption.

- Distribution. We consider whether the approach provides facilities for distributed service implementation in a seamless fashion, without adding complexity to the business code.

- A posteriori development. We consider the approach enables for the implementation of monitoring a posteriori. Therefore, we are concerned with the difficulties involved for turning an existing non-monitorable implementation into a monitorable one. 


\subsection{Chapter Summary}

In this chapter, we presented how the Services Provider Library and Services Consumer Library extend the Service Monitoring Framework presented in the Chapter 3. The libraries provide practical facilities and concrete class implementations for representing and managing operations and notifications, for both provider and consumer perspectives. We have discussed particular implementation details for monitoring SOA implementations and we also discussed how the framework and library could be extended to improve its usage in specific scenarios such as workflow-based applications. In this chapter, we have finished our explanation about the technical details of design and implementation of the execution progress monitoring concern. The next chapter zooms out by exploring the effects of the monitoring concern on the overall software development process, besides design and implementation details. 



\section{CHAPTER 5}

\section{Impact on Software Processes}

Previous chapters have focused on the design and implementation activity of monitorable services development process. Unfortunately, the crosscutting nature of the monitoring problem is not limited to classes and methods. In fact, its crosscutting nature goes beyond the design and implementation activity. The monitoring concern crosscuts almost every software development process phase, from software inception, passing through requirements elicitation, to validation and deployment. To some extent, it can even affect project management and planning, as it requires some considerations during the planning activities of estimating durations, costs and allocated resources.

This chapter complements previous ones by discussing the impact of developing monitorable services on the context of a software development process. It is not the intent to design an adapted software process for supporting monitoring, based on some widely adopted software process, such as RUP, XP or Scrum [Rat99a, Rat99b, XP09, Sch95, Raw03]. This would lead to a specific software process definition, whereas it is a known fact that there is no "ideal" software process, and it is very common that organizations need to adapt, improve and create specific processes that are adequate for their organization-specific characteristics. Therefore, instead, we discuss general software process disciplines, phases and activities, in order to provide the tools and guidelines that enable one to adapt virtually any software development process that is based on standard disciplines.

Before we start to discuss the impact of monitoring on the software process disciplines, let us review the software process literature and the fundamental concepts involved.

\subsection{Software Processes}

A software process is a set of activities that lead to the production of a software product [Som07]. Depending on the type of software that an organization intends to develop, its objectives and characteristics, people involved, available time, budget and business-specific considerations, the activities involved may differ a lot from one software process to another. For example, these activities may involve the development of software from scratch, in a standard programming language, or instead involve extending and modifying existing systems, or still involve configuring and integrating off-the-shelf software or system components.

For now, it is not possible to extensively automate software development processes, and it seems probable that it will remain so, at least in the next few years [Som07]. The reason is that software processes are complex and rely on people making decisions and judgements, like all intellectual and creative processes. Despite the challenges involved, Process-centered Software Engineering Environments (PSEEs) [PKG95] enable users to explicitly define and model the processes, supporting the activities of defining, monitoring and evaluating them. Such environ- 
ments usually automate part of the process and provide guidance to the development team. In addition, Computer-aided software engineering (CASE) tools can support some process activities, by automating operational activities and providing facilities for generating documentations and organizing artifacts, but they do not substitute or automate human creativity and judgement upon the software process. Combined, PSEEs and CASE tools represent remarkable advances towards software process automation, although it is still not possible to achieve complete automation throughout the entire project life-cycle.

There is an immense diversity of software processes. The reason is that there is no ideal process and thus many organizations have developed their own approach to software development, one that exploits the team in the organization and considers specific characteristics of the systems being developed. For example, a very structured development process is crucial for critical systems, while agile processes are likely to be more effective for business systems with rapidly changing requirements.

Although there are many software processes, and many differences involved (specific activities, the order on which they occur, the time spent on each one of them, their recurrence, different approaches to handle the same activities, etc.), still we identify some fundamental activity classes that are common to all software processes:

- Software specification. Software functionality and constraints on its operation are defined. The objectives and how they are supposed to be achieved in terms of software functionalities, requirements and use-cases become clear.

- Software design and implementation. The software itself is produced to meet the specifications and achieve the objectives defined.

- Software validation. The software is validated in order to ensure that it fulfills customer needs.

- Software evolution. The software evolves to meet changing customer needs.

All of these fundamental activity classes are influenced by the monitoring requirement. In the next sections, we discuss these activities in more detail and then we discuss how they should be adapted to consider the monitoring requirement.

Despite the fact that many organizations create their own approach to software development by creating their own software process, which in theory should be ideal for the organization considering it has been created for its specific needs, there is often scope for improving the software process. The reason is that they may include outdated techniques or may not take advantage of the best practice in industrial software engineering. Indeed, many organizations still do not take advantage of software engineering methods in their software development [Som07]. Reducing the diversity of software processes across an organization by enabling use of process standardization leads to improved communication, reduction in training time and makes automated process support more economical. Thus, standardization is an important step in introducing new software engineering methods and techniques. Therefore, by the time we need to adapt software processes and activities to consider the monitoring requirement and properly handle its particular characteristics, it is important for us to define standardized and regular activities for dealing with changes in the software processes. 


\subsection{Process activities}

The four basic activities of specification, design and implementation, validation and evolution are organized differently in different development processes. The details on how these activities are carried out depends on the organizational structure involved, people and type of software. Therefore, there is no wrong or right way of organizing process activities.

In this section, we focus on the activities themselves, rather than on the sequence in which they are organized or on the role they play in the process model to which they belong.

\subsubsection{Software specification}

Software specification, or requirements engineering, is a crucial phase of the development process. It includes the process of understanding and defining the required services for the system, identifying constraints and specific business characteristics that should be considered for the project to succeed. It is crucial because errors at this early stage lead to later problems in the system design and implementation.

Figure 5.1 shows an example of a requirements engineering process. This process outputs a requirements document, which is a specification for the system. There are two levels of detail for describing requirements: higher-level descriptions that are useful to end-users and customers; and detailed and more structured descriptions that are useful to developers.

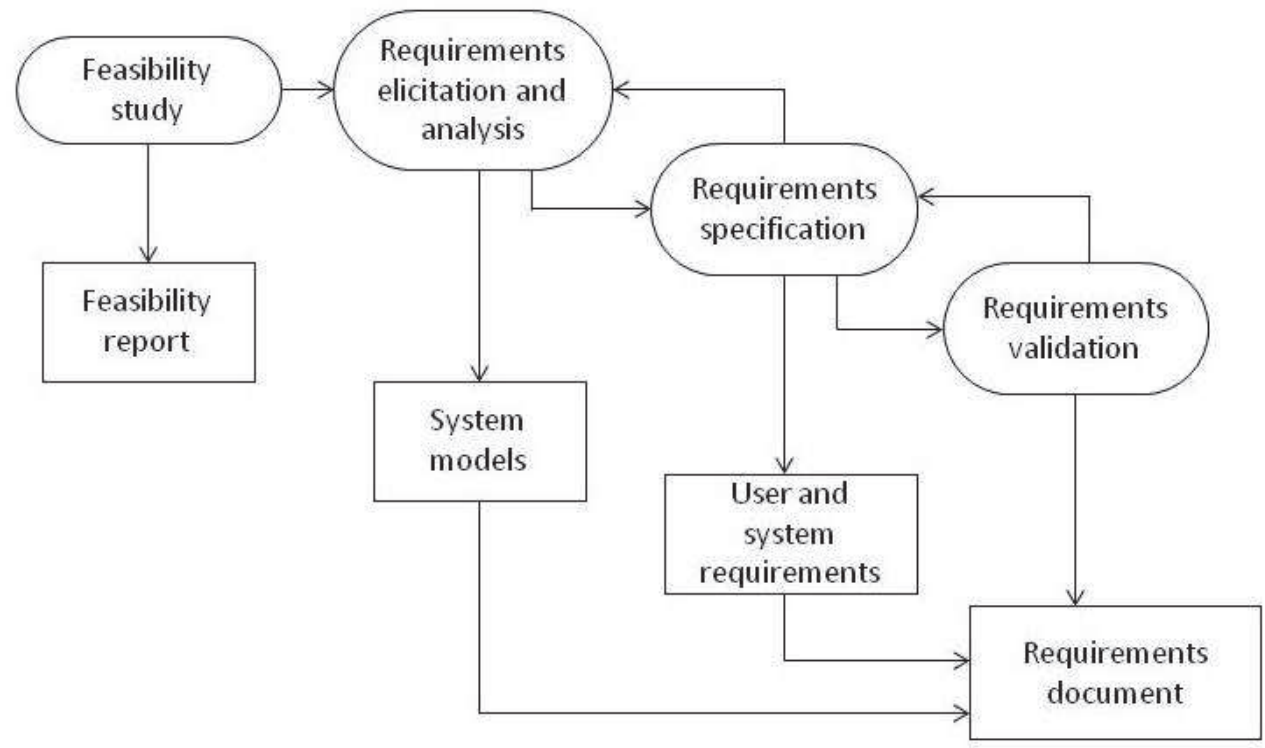

Figure 5.1 Requirements engineering process [Som07].

There are four main phases in a requirements engineering process:

- Feasibility study. In this phase, the user needs are analyzed in order to determine whether the project is cost-effective and fits the budgetary constraints of the organization. Estimates are made for the total project effort and investments required. Estimates are objectively compared to the project benefits, supporting project decision. 
- Requirements elicitation and analysis. This phase involves observing existing systems, current processes and discussing with potential users. The goal is to understand the system to be specified.

- Requirements specification. The activity of translating the results of understanding the user needs into a document that defines a set of requirements. Two types of requirements may be included in this document. Both user requirements and system requirements are specified. The first describes the requirements from an end-user perspective, while the other provides a more detailed description of the functionality to be provided.

- Requirements validation. It involves checking the specified requirements for realism, consistency and completeness. Errors of understanding or specification are expected to be discovered and should be corrected before design and implementation begins.

In practice, the activities in a requirements engineering process are not a strict sequence. Instead, they are interleaved, because requirements analysis continues during definition and specification, and new requirements appear throughout the process.

\subsubsection{Software design and implementation}

In this stage of the software process, the requirements specifications produced in previous stage is converted into a concrete and executable system that meets user needs. A system design is a description of the structure of the software to be implemented, providing details on the data that are part of the system, the components, their interfaces and how they interact with each other. It involves developing models of the system at different levels of abstraction. As the design is decomposed, errors and omissions might be discovered and then it should feed back to earlier stages of the design process to allow for improving models. Feedback from one stage to another and consequent redesign and rework is inevitable in all design processes. Figure 5.2 describes a general model of a software design process.

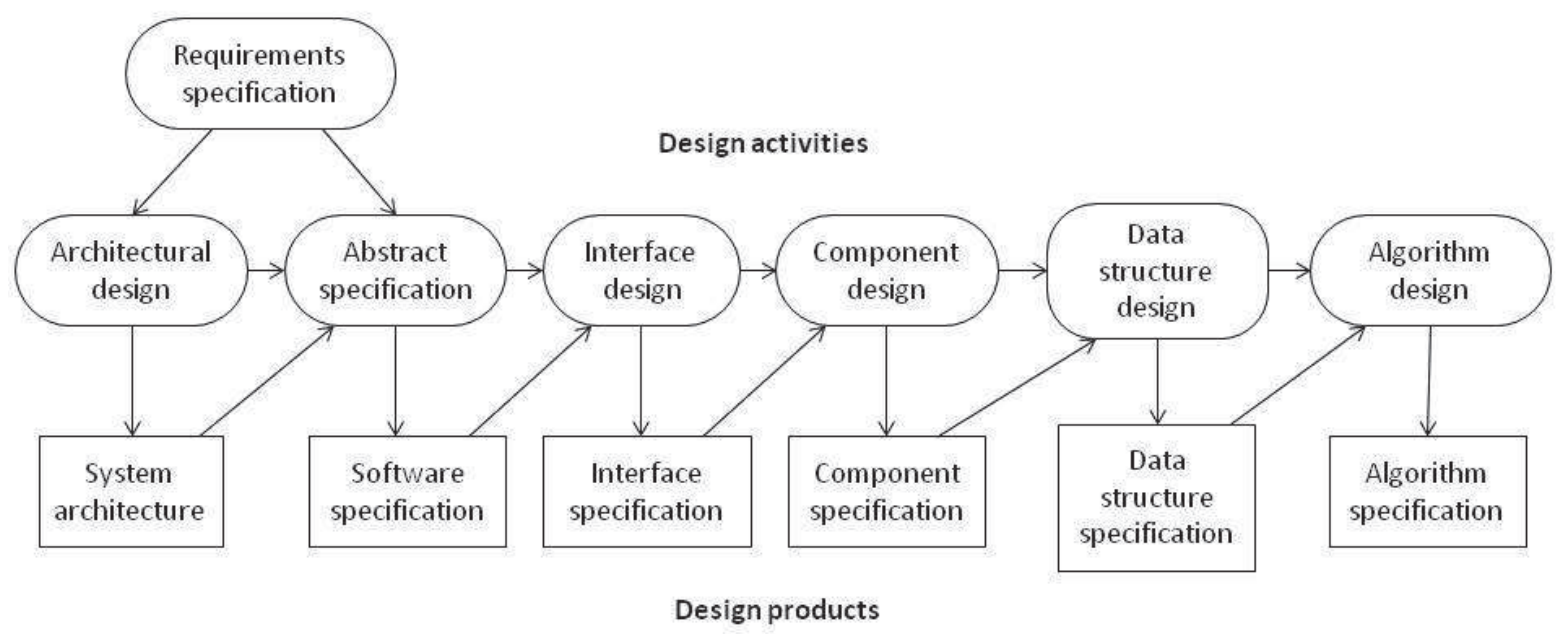

Figure 5.2 General model of design process [Som07]. 
The results of a design process are artifacts that provide details on the data structures and how the system components should interact in order to meet requirements. As the design process advances, the artifacts become more detailed. The final results are precise specifications of the software to be implemented, sometimes including specifications of algorithms to be implemented.

Some typical activities of a general design process are:

- Architectural design. The higher level sub-systems and their relationships are identified and documented.

- Abstract specification. The services of each sub-system and their constraints are specified.

- Interface design. For each sub-system, the interfaces with other sub-systems is designed and documented.

- Component design. Services are decomposed into components and the interfaces of these components are designed.

- Data structure design. Data structures used in implementation are designed in detail and specified.

- Algorithm design. Algorithms used to implement services are designed in detail and specified.

In practice, real processes may adapt these activities in many different ways, whether suppressing algorithm design or interleaving some of the last stages of design with the implementation process. In agile methods of development, the outputs of design process are represented in programming code rather than in separate specification documents [GCS07].

A widely used approach for representing the outputs of a design process is by using structured methods such as the Unified Modeling Language (UML). This approach relies on producing graphical models of the system and, in many cases, allows automatic generation of code from these models.

\subsubsection{Software validation}

The software validation phase is intended to show that a system conforms to its specification and that the system meets the expectations of the customer buying the system. It involves checking all activities in the software process, but the major effort often occurs after implementation, when the system is tested under operation.

The stages in a general testing process are:

- Component (or unit) testing. Individual components are tested to ensure that they operate correctly. Components are tested independently, without other system components, with fictitious data and out of the context of an integrated system.

- System testing. The components are integrated to make up the system. The system is validated to whether it meets the functional and non-functional requirements specified. 
- Acceptance testing. The system is tested with data supplied by the system customer rather than simulated test data. The real data may exercise the system in different ways from the test data and errors in the specifications may be found. This is the final stage in the testing process before the system is accepted for operational use.

As a test case fails, failure information is fed back to earlier stages in order for the implementation, design or specification documents to be corrected. Figure 5.3 shows a general test process model.

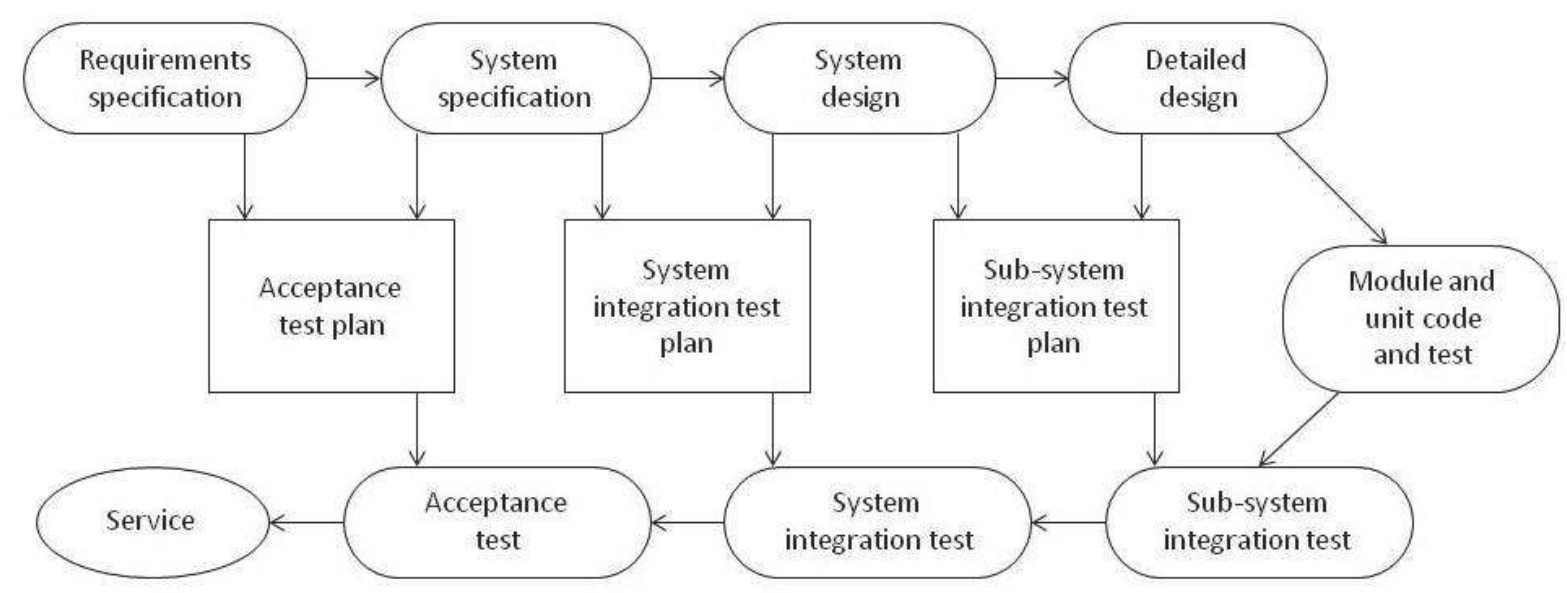

Figure 5.3 General test process [Som07].

\subsubsection{Software evolution}

When a system passes all acceptance tests and is finally delivered to the customer, the software life-cycle is still not finished. User needs change over time, due to external changes of business or to different organization contexts, people involved and goals. Even when there is no obvious change in the customer scenario, the fact that he has already started using a system version provides him expertise for understanding his own problem and thinking of new functionalities or changes to existing ones that would improve the system.

Because of these changes, software maintenance and evolution processes are very important for the system to remain useful as time passes by. In fact, the costs of maintenance are often several times the initial development costs.

Software development and maintenance are not completely distinct processes. Instead, they are a continuum, because software is continually changed over its lifetime in response to changing requirements and customer needs. Figure 5.4 shows the evolution process.

\subsection{Impact of service execution monitoring on software processes}

Previous sections have provided an overview of general software processes, by discussing their involved activities and core concerns. This section focuses on how these processes are affected by the monitoring requirement, in order to obtain full systematic considerations throughout the software development. 


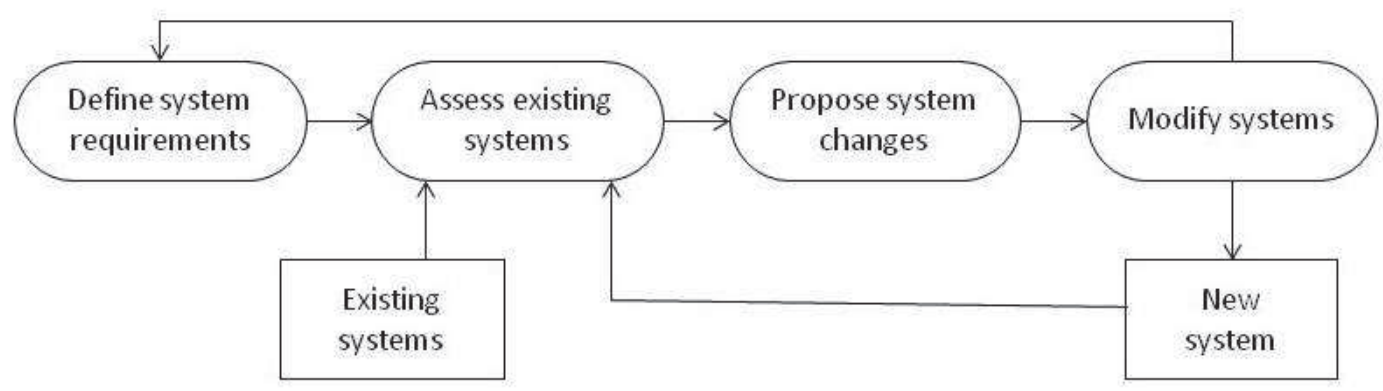

Figure 5.4 System evolution [Som07].

The implementation of monitoring requirement is a project decision that often involves subjectiveness, because its importance also depends on human perceptions and opinions. In this section, we assume the monitoring requirement as an already made project decision, in the context of a software development with enough complexity for justifying the systematic reuse of an existing monitoring framework such as the one proposed in this work, along with patterns and process guidelines.

In addition, one can inquire whether it is actually important to consider the monitoring requirement in all activities of a software process, instead of concentrating solely on the design and implementation activity. In fact, one can ignore the monitoring details in the early stage of the development process, by leaving it entirely for the design and implementation activities, such as we demonstrate in the case study presented in Chapter 6. However, if one intends to use a more formal approach and generate more detailed documentations throughout the entire development process, anticipating concerns and formalizing expectations, then it is important to consider some additional concerns regarding the monitoring requirement. In this section, we assume the latter case, by suggesting possible adaptations throughout the development process.

Although we intend to provide generic guidelines for considering the monitoring requirement on generic processes, we use RUP-specific artifacts to provide concrete examples, for didactic purposes, whereas the principles could be applied to any given process. We organize the subsections according to four affected process activities: specification, design and implementation, validation and evolution.

\subsubsection{Software specification}

Software specification is a crucial activity in the software process. It includes understanding and defining the required services for the system, establishing and documenting inputs, outputs and expected behavior of system functionalities. There are many different techniques that may be applied in order to identify software requirements, gather information about them and create a concrete list of use-cases and constraints in a documentation: interviewing, observing user current environment and processes, observing existing systems, prototyping, etc.

This activity outputs everything that is expected from the system, making it clear and well documented, and serving as a contract between the customer and developers. The process of elicitation and analysis should not ignore the details of the service monitoring requirement. Therefore, with respect to monitoring, the outputs of the requirements specification process 
should include:

- A clear identification of which services should support execution monitoring.

- The priority of monitorable requirement in comparison to other project requirements.

- For each desirable monitorable service, the detailed functionality workflow, including operation costs and a clear explanation of the criteria used for establishing the costs.

In theory, if it had no implications to project estimates, costs and schedule, it would be clear that every single service in the system should be designed to support monitoring. Unfortunately, this is not the case, as discussed in previous chapters, despite the use of a framework and libraries that provides some facilities and minimizes required effort, we still cannot ignore the fact that implementing this requirement adds complexity and increases project required effort.

Therefore, it is important to clearly identify which use-cases and system functionalities are likely to support execution monitoring, and categorize them to distinguish between the ones where monitoring is simply desirable from those where monitoring support is critical.

For example, services that involve network traffic or streaming (such as downloading, uploading or software installation) usually have monitoring support as a critical requirement and almost as important as the functionality itself being monitored.

Even when one decides not to implement the monitoring support in first increments and releases, due to the tight schedule or the low priority assigned to the monitoring requirement, still it is important to determine whether it will be implemented in the future, in order to choose a design and architecture that favor the monitoring implementation.

The criteria used to determine whether a service execution should or should not be monitorable is likely to be a subjective matter. The value/importance given to the monitoring requirement depends upon user perspectives. Subjectiveness apart, there are some criteria that can be used to support this decision:

- A measure of the additional effort and increase of complexity required to implement monitoring. The required effort depends on the complexity of the service workflow. The amount of operations and suboperations involved should be very clearly specified to enable analyzing the effort needed to support monitoring.

- The expected service usage frequency. Since the progress feedback is a matter of improving human-computer interaction, and does not directly contribute to the service well functioning, the frequency of the interactions matters. Depending on how often and how many users consume the service, despite the importance of the service in the system as a whole, it may help on deciding if monitoring is really needed for the service.

- The expected time for the service execution. If a service execution duration is expected to be too short because it involves only trivial and fast operations, then it reduces the importance of monitoring.

That said, a systematic approach towards identifying which services should have monitoring capabilities can be divided in two distinct alternatives: 
- Identification a priori. Identify which services should be monitorable early in the requirements engineering process, before the use-cases and functionalities are actually specified with detailed activity workflows. This identification represents more truly the real desire of users, independent of feasibility due to required effort or complexity. It can be systematically performed by interviewing users about every service of the system and taking note of their expectations. In the end of the interviews, one can calculate a score representing the more desirable monitorable services according to user preliminary expectations. The services can be sorted out based on this score which results on a list of likely monitorable services, to be considered in order of priority.

- Identification a posteriori. Identify desired monitorable services in the end of the requirements engineering process, after all use-cases and functionalities are specified in details. Analyze the workflows involved in the specified services and use them to make more precise estimates of the required effort, expected execution duration, and usage frequency. Use these measures to calculate a score for each of the activities and sort the more likely to become monitorable services.

One can provide weights for both scores (a priori and a posteriori) and combine them to find a final score which can be used to finally determine which services should be considered for monitoring.

In RUP, Use-Case Models are used to model the system intended functions and their environment, and serve as a contract between the customer and the developers [Rat99a]. According to RUP specification, the typical properties of a Use-Case Model are: Introduction; Survey Description; Use-Case Packages; Use Cases; Actors; Relationships; Diagrams; and Use-Case View. In addition to these typical properties, Use-Case Models could be adapted to explicitly mark the use-cases that are likely to be monitorable, as shown in Figure 5.5, an example usecase diagram for a Course Registration System, in which the use-cases that involve monitoring are painted in green and marked with the "«Monitorable»" stereotype.

Note that the actors representing external systems are also marked as monitorable, because there are monitorable use-cases that depend on them. This is an optimal scenario, leading to detailed and more accurate monitoring results. However, having the dependent external systems monitorable is not mandatory for implementing a monitorable use-case, since it is still possible to consider the external system action as a single operation in the operations tree, abruptly changing progress state from $0 \%$ to $100 \%$. Such behavior would only compromise the monitoring quality if the external system call happens to represent most part of the overall service execution.

Identifying the monitorable use-cases on an early stage of the software process and explicitly representing them through use-case diagrams will help on providing a global view of the monitoring impact on the software as a whole, the amount of monitorable use-cases and their relationships, helping on planning and making this information very clear for the design and implementation stage of the software process. For example, such a global view is important for a component-based development process, since the search for reusable components should carefully consider monitorable components for implementing monitorable use-cases.

Despite the use of UML diagrams such as state diagrams, use-case and activity diagrams, requirements specification often make use of natural language as a basis for registering knowledge. By using a structured format (such as stereotypes and structured fields) for organizing 


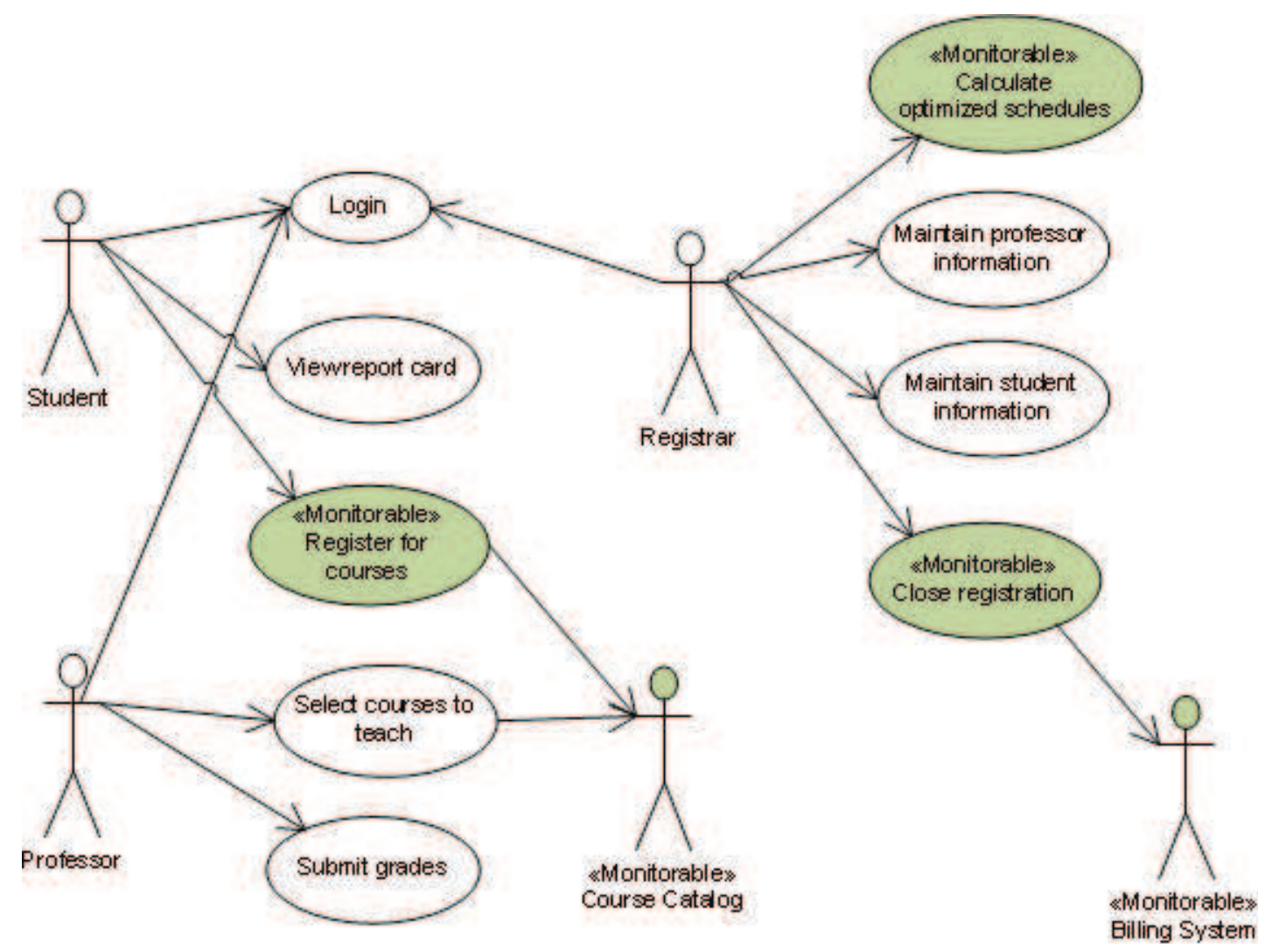

Figure 5.5 Main use-case diagram for a Course Registration System, adapted to make monitoring explicit.

monitoring-related information, instead of natural language, we intend to reduce ambiguity and stimulate standardization for the software documentation. One can inquire whether this extra effort to give evidence to monitorable use-cases is actually worth the benefits. In fact, providing such particular evidence is a decision to distinguish the monitoring requirement from other non-functional requirements. Therefore, such a decision is only reasonable if the monitoring requirement is considered more critical when compared to other non-functional requirements. Otherwise, the other non-functional requirements should be privileged for evidence.

Marking monitorable use-cases on an early stage of a use-case diagram specification provides an interesting overview of how monitoring can be spread throughout the system, considering user preliminary intentions (a priori), but it does not provide the necessary monitoring information to support their implementation or to serve as basis for a systematic analysis of the actual importance of monitoring for each use-case, considering their expected execution time, their complexity, their dependencies, and the required additional implementation effort. In order to gather these monitoring information, we would propose some changes to the typical use-case properties documented in Use-Case Reports defined by RUP. The original use-case properties specified in RUP are: Brief description; Flows of events; Relationships; Activity and State diagrams; Use-case diagrams; Special requirements; Preconditions; Postconditions; and Other diagrams.

The specification of monitorable services should include some information that is crucial for implementing monitoring support in system services. It is important to detail every oper- 
ation and suboperation that take place in the service execution and their relative costs in the overall execution. If the monitorable services identification considers only a priori analysis, then the costs should be determined during the process of specifying and detailing the workflows involved in the monitorable service. On the other hand, if the identification is performed $a$ posteriori, the services are already specified by the time they are determined to be monitorable, and the specification should be revised in order to consider monitoring information.

The general purpose nature of RUP makes its artifact descriptions very generic, in order to fit for any kind of software development. The Special requirements and Other diagram sections of the standard Use-Case Report enable virtually any kind of information to be considered on use-case specifications. In fact, this work makes use of such generic sections for specifying monitoring-related information. In this context, we propose a specification pattern that is intended to cause minimal impact on the original use-case properties defined in RUP. We suggest a structured format for registering monitoring information, by adapting two sections of the standard RUP Use-Case Report: Special Requirements and Activity and State diagrams.

We use the Special Requirements generic section of the Use-Case Report to characterize the use-case with regard to the monitoring requirement. In this section of the report, the following questions need to be answered:

- Is monitoring desirable a priori? It is important to determine whether the customers and end-users actually consider monitoring relevant for the use-case. This aligns expectations and makes it easier to cut the monitoring requirement from the project, once it is known to be of low importance to the end-users.

- How often will the use-case be executed? The impact of removing the monitoring requirement can be assessed by analyzing the expected frequency of use-case execution and how many end-users would be prejudiced. This information will complement the previous question, because it may happen that a use-case is used by only one single person and once a year, but still he can consider it a very important requirement when analyzing the use-case isolated from the context.

- How complex is it to implement monitoring? Is the basic flow significantly nondeterministic? Deterministic activities are easier to design and implement the monitoring requirement, whereas non-deterministic ones makes monitoring implementation more complex and thus more costly. One can inquire whether it is actually feasible to answer this question in such an early stage of a development process. In fact, the idea is only to provide a preliminary analysis based on the use-case nature. For example, a streamingrelated use case, such as a file download, is often deterministic and simple to implement monitoring.

- How long is the basic flow expected to run? Long-running operations are probable candidates to support monitoring, because they obviously amplify the benefits of being monitorable, whilst short-running ones are less likely to become monitorable because there is no time for receiving monitoring feedback as the execution ends too fast.

- Does it depend on third-party components or systems? Do they enable monitoring? In order for a use-case to support monitoring, all of its involved activities - or at least the most cost-representative of them — should also support monitoring. Therefore, if the 
use-case main flow of event depends on a third-party component or integration with other system, this one should also provide monitoring support.

- Is monitoring considered for future implementation? Even if monitoring support is not actually implemented for first releases, it is important to prepare the software architecture and design for a possible future implementation.

- What are the involved operations and their monitoring costs? This is answered by using an adapted use-case activity diagram which provides costs for each activity. Further, we cover this subject in more details.

- What is the criteria used for establishing the activity costs? It is important to apply a clear criteria and method for assigning the costs of each activity, in order to assure regularity between the activity costs, specially when different people assign the costs of the activities. In addition, formalizing and documenting the criteria is crucial for future maintenance and software evolution.

Activity diagrams help on graphically describing the flow of events of a use-case. It captures workflow in an organization and shows action states, transitions, decisions, concurrent operations, etc. From the monitoring requirement perspective, the use of activity diagrams is suitable to answer the question regarding operations and their costs, because the activities in the diagrams map into the operation concept of monitorable services, and it is possible to adopt a systematic approach for associating the costs of each activity in the activity diagrams.

Figure 5.6 shows the steps of the Register for Courses use-case, an example extracted from the original RUP material [Rat99a] and adapted to include the estimated execution costs for each activity composing the workflow. What differs the adapted activity diagram from a typical activity diagram is the existence of labels associating costs to the activities.

Note that use-cases may provide more than one flow of events. In an activity diagram, a flow of event is a path linking an initial state to a final state. In the example shown, there are two different flow of events. Therefore, there is no way of determining the total cost of a usecase, due to the fact that the costs change depending on the flow of events (scenario). Thus, the adapted diagram shows a label associated to the diagram initial state which provides the set of possible costs (one cost for each possible flow of events). The cost of a flow of events is given by the sum of the activities within it. These costs are only intended for the alignment of expectations between the requirement analysts and developers, although they are subject to changes during the design and implementation stage.

Use-case activity diagrams must focus on higher-level functional workflows. They are not supposed to specify the implementation details and algorithms. Instead, they are intended to serve as a high-level specification for the design and implementation stage. Put simply, they are solely an input for the developers to broadly understand what is expected. That said, it is important not to misunderstand this concept, being careful not to dirty the activity diagrams with implementation-specific concerns. For example, the following implementation-specific concerns should not be included in use-case activity diagrams:

- Providing progress feedback. During execution, current progress changes in every transition from one activity/state to another, increasing the cost of the originating activity of the transition. Thus, from the implementation perspective, in between such a transition, a progress feedback event should be performed. 


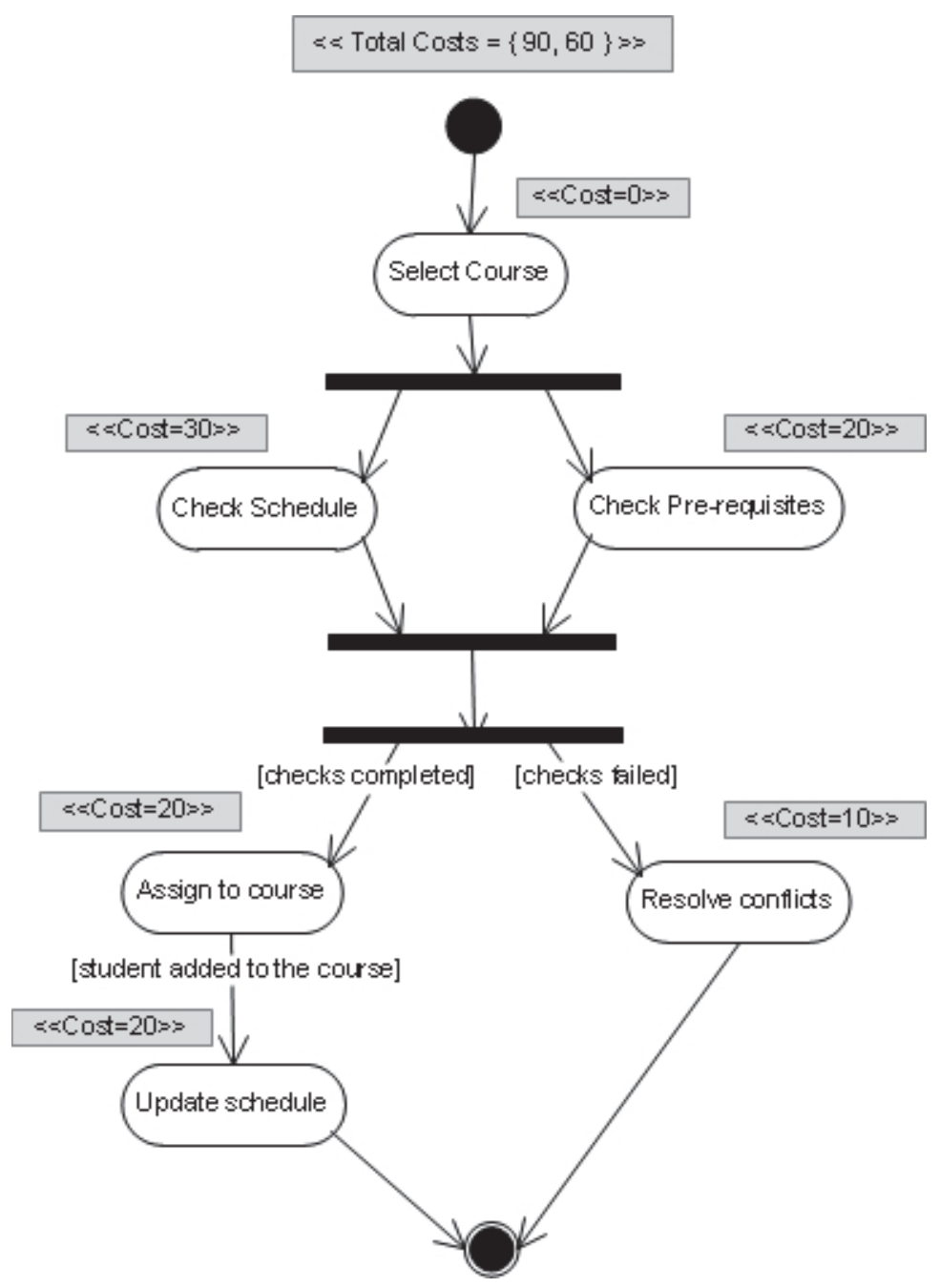

Figure 5.6 Activity diagram for the Register for Courses use-case, including execution costs.

- Acknowledging cancelation request. From the implementation perspective, if the usecase represents a cancelable service, some activity transitions should verify cancelation request, and rollback the on-going operations if it is the case.

- Calculating total cost. From the implementation perspective, the very first action in a use-case flow of event to be implemented should be the calculation of the total execution cost. When the exact flow of event cannot be predicted at first, still it needs to adopt a strategy for establishing a reference cost — such as using the highest possible cost based on the worst-case flow of events, which guarantees that the progress percentage never retrocede. In addition, it is also possible that an activity cost is not a constant value, but rather a context or input-dependent variable that needs to be dynamically calculated at run-time.

These implementation-specific actions should be ignored during the requirement specification stage. However, they must be considered during the design and implementation stage of the software development process. Section 2.5 provided some examples on how to transform a given activity diagram into one that makes these implementation-specific details explicit. 
Furthermore, some activities can be described on a high-level of abstraction, such that they are not actually atomic activities, but a simplification of another workflow. In such cases, the activities are themselves monitorable and their inner workflow should be unfolded in order to establish the detailed costs. Otherwise, the progress could loose uniformity and consistency.

Once the monitoring questions are properly answered, it is possible to make more precise estimates of the effort required to implement monitoring on the services and this information will lead to the final decision of whether it is worth to implement monitoring on the service. Specifying monitorable services provides inputs for the project feasibility study, to support the decision of whether or not to implement monitoring and finally provide systematic information for the design and implementation stage of software development, which is covered in the next section.

\subsubsection{Software design and implementation}

Even though one decides that the first iterations / deliverables of the system will not provide support for monitoring, because of schedule or budget constraints, still it is important to consider whether monitoring is intended to be considered in the future. Since the system design and architecture should be compatible with the monitoring requirements, it is important for minimizing project refactoring and rework, while avoiding loss of compatibility with existing service consumers. That is, the services should not change their signatures, inputs and outputs, by the time they evolve to support execution monitoring.

Supporting service execution monitoring requires using some design patterns and standards all along the application, in order for the monitoring code to remain regular, uniform, and easy to maintain. With respect to monitoring design and implementation, most details and concerns have been covered in Chapters 3 and 4. In the design and implementation stage of software development process, the requirements specifications are used as basis to guide the software production. As a result, the monitoring-related information will be used to guide the implementation of monitorable services.

If the requirements specifications are complete and contain detailed information regarding monitoring, along with activity diagrams and assigned costs, one could inquire whether the implementation of monitoring for each use-cases should be a trivial and semi-automated task, by simply converting the monitoring activities defined in the diagrams into code for creating operations and assigning costs. Put simply, all the designers and developers would need to do would be to apply what has been already defined in the specifications. However, this is often not true, due to the following reasons:

- Use-case activity diagrams only provide the higher-level activities from a business perspective. The actual implementation of a use-case unfolds the activity diagram activities into more specific activities. The gap between an activity diagrams and the actual classes and methods involved in the implementation can be huge. Therefore, the activity diagram specification will serve only for the development guidance, documentation and validation.

- Costs of activities assigned during the requirements specification stage are often rough estimates. The actual costs of the operations will depend upon the specific algorithms, components and implementation strategies used. Therefore, the developer will need to adjust the costs while the activities are unfolded and actually implemented. 
One can also inquire whether the monitoring requirement should affect the diagrams and other documentations that are elaborated in the design and implementation stage of the software process, such as sequence and collaboration diagrams. In an architectural perspective, the designer can assume that a framework to support monitoring is already instantiated. In such case, the software design specification would only need to consider the framework usage, so that calls to the framework methods should conform to a predefined pattern.

If one intends to be strictly rigorous regarding specifications, then every single diagram related to a monitorable use-case should explicitly specify the monitoring-specific behavior. In such case, sequence diagrams would need to make the monitoring-related method calls explicit for every monitorable service execution. This rigorous approach has the advantage of minimizing the risk of misinterpretation from the developers and deviation from the expected standards. On the other hand, it would lead to a drastic increase in the sequence diagrams of such monitorable services, since each single arrow pointing from a consumer to a provider would result in many callback arrows of asynchronous progress feedback, not to mention the increase of the diagram complexity, affecting legibility by mixing the monitoring-related calls with the core business flow calls.

Therefore, we suggest an in-between approach for monitorable use case design specifications. Since the monitoring framework usage assumes the same pattern for every monitorable use-case, the designer can choose only one specific monitorable use-case to serve as reference to the others. In such use-case, all the diagrams would make the monitoring-related method calls explicit. In an architectural diagram, the framework would appear as a dependency. On a sequence diagram, all the callback methods for sending progress feedback would be made explicit. Once this use-case associated diagrams are specified, we would have sufficient templates for the development team to understand the monitoring pattern. Therefore, for every other monitorable use-case, it would be possible to hide the monitoring-related method calls. Furthermore, each arrow representing a monitorable service call could have the service name underlined or highlighted in order to denote that the given service must provide monitoring feedback.

With regards to the class diagrams, no special consideration is needed, since the monitoring impact is naturally represented as long as the service providers need to implement the IMonitorableServicesProvider interface defined in the framework such as explained in Chapter 3. In addition, monitorable services will implement a specific method for retrieving an IServiceMonitor.

\subsubsection{Software validation}

The software validation activity aims at verifying whether the software product conforms to the specification and thus satisfies user expectations before it is actually deployed and delivered into the production environment. In this context, once monitorable services have been developed, the quality of monitoring results should be verified towards uniformity and consistency. The calculated estimates of service execution duration should be verified and compared to the actual execution time.

Since the operations cost assignment is not an automated task and depends upon human judgement, it is very probable that the resulting monitoring information - along with the calculated remaining time estimates - is not consistent and uniform at the very first service execution. 
One can use a systematic approach for the consistency verification, by collecting samples of the calculated estimates for the remaining execution time along the first seconds of execution, and then comparing the sampling average to the actual measured execution time. If the deviation between estimates and the actual cost is greater than a certain defined threshold, than one can conclude that there is a consistency problem with the monitoring results, since the remaining time estimates calculation assumes the consistency of the progress feedback increase rate.

If the resulting progress consistency fails in the consistency check, there are two possible reasons — not necessarily exclusive — for this:

- The assigned costs are not time-consistent. Progress feedback consistency depends solely upon the costs assigned to each operation in the monitorable service execution chain. Time-consistent operation costs should be assigned on a proportional time-consuming criteria: if an operation is twice longer than the other, then it should be assigned twice the other cost. In practice, it is often difficult to assign accurate time-consistent costs during the implementation, because it is not trivial to determine how long would it take for each operation to execute in comparison with the others. Once the inconsistency is identified, the assigned costs need to be reviewed and tuned. We suggest a systematic process for tuning the operation's costs, based on the use of a profiling tool [EQA11, Cor10b, MW07]. A profiler will track the time spent on each one of the methods and count how many times each method has been called. As an operation consists mainly of method calls, one can sum up the actual time spent on the execution of each operation. Then, the least time-consuming operation can be arbitrarily assigned a cost of $x$, while every other operation will be assigned a cost of $\mathrm{k}^{*} \mathrm{x}$, keeping the proportion with regard to time.

- There are abrupt progress increases within the operations. A perfectly uniform progress feedback should simulate an apparent continuity during the service execution. On the other hand, by definition, the framework is based on discrete progress notifications that are occasionally updated. Strictly, the last notified progress state is always outdated when compared to the actual progress state, because even the last received progress notification represents a given snapshot of the past, even for those monitoring results supposedly consistent. In practice, this characteristic does not become a consistency problem if the intervals between progress notifications are sufficiently short. An operation is said to be atomic, with respect to the progress feedback, when it does not providing partial progress notifications while it executes. As an effect, such atomic operations simply change progress state from zero to a hundred percent. Therefore, if an atomic operation is assigned with high relative cost when compared to the overall monitorable service cost, this will result on abrupt overall progress changes. If such atomic operations are timeconsuming, the last received progress notification becomes too outdated and cannot be used to calculated remaining time estimates because it would generate distortions. If the assigned costs are supposedly consistent (and verified by profiling tools), then the atomic operations should be revised in an attempt to split its execution to provide partial progress feedback.

The verification of whether the monitoring results are actually consistent should be performed by measuring execution on different inputs/scenarios, in order to find a better correspondence between the assigned costs and the input variables. There are cases where costs of 
operations cannot be statically assigned and need to be calculated at run-time according to the service inputs.

Different execution environments can lead to different execution times, depending on the criteria used to determine the weights of activities. Thus, although the monitoring may have seemed to work properly on the development environment, running under certain hardware configurations, it may still not work as expected on the user production environment, with different characteristics. In such case, the criteria and costs should be revised to work regularly and uniformly, according to the environment configuration.

\subsubsection{Software evolution}

The software development life-cycle is not finished when it is delivered to the customer. Since user needs change over time, software must be adapted to new demands, due to internal or external changes. Changes to the software specification is often requested. Even the simpler changes to the implementation of the software should be analyzed to whether it could impact execution monitoring. Changes can occur directly on the monitorable service implementation or indirectly on the components on which the monitorable services depend. In both cases, costs and operations should be revised and verified towards consistency. Even when the overall functionality and output is not affected by the change, the fact that the execution flow changed can affect the logical organization of operations and suboperations under consideration, and execution time of one operation in comparison to others, and therefore the costs should need to be reviewed and re-calibrated.

Before changes are actually implemented, the specifications should also be reviewed, including the use-case reports, activity diagrams, and monitoring-related information. The documented criteria used to determine the costs should be carefully reviewed in order to add new activities or make changes to existing ones.

In summary, with respect to the software evolution stage of a general software process, there are few concerns to add with regard to monitoring, except to keep revising the operations and assigned costs in order to preserve consistency.

\subsection{Chapter Summary}

In this chapter, it has been presented a review of software processes fundamentals with a particular focus on the Rational Unified Process (RUP). In addition, the characteristics and activities involved in general software processes have been discussed. Then, each one of the activities is discussed with respect to the monitoring requirement, including the impact on the process and introducing some guidelines for a systematic monitoring consideration. 



\section{CHAPTER 6 Work Evaluation}

In previous chapters, we presented the implementation details of a solution consisting of a framework and two libraries designed for supporting and improving the development of monitorable system services. In this chapter, we describe a case study involving the framework and library usage in the context of a software project, in order to provide an initial evaluation of the proposed solution. This chapter is organized as follows: Section 6.1 defines this case study; in Section 6.2 the planning of the case study takes place; then, Section 6.3 shows the analysis and interpretation of the results; finally, Section 6.4 summarizes this chapter.

\subsection{Definition}

\subsubsection{Context}

During the months of July and August 2011, we performed a case study at SUATI - Suporte Avançado em Tecnologia da Informação - , a software development company based in Recife, in order to evaluate the effectiveness of the developed solution. The company has nearly 6 years of experience on the development of software systems. The project in the context of which the case study took place consists of more than 500k lines of code (LOCs). The company development team is organized in four autonomous development cells, each one containing a leader and nearly 8 software developers. One of the cells was about to start the development of a new system module where the monitoring requirement was considered important, because the project involved complex risk analysis algorithms, time-consuming simulations of financial scenarios and database-intensive operations, which take significant time to execute. Put simply, with respect to the monitoring requirement, the final goal was to enable the display of a percent-done indicator along with the remaining execution time estimates during the risk analysis simulation. The development team had both novice and experienced senior developers and both would need to implement monitorable components that provide part of the overall functionality. This enabled us to measure implementation efficiency for both experienced and novice developers. The development scenario at SUATI suited for the case study because it involved a large system, involving distribution, concurrency and cross-technology communication (COM, .NET, SOAP, etc.) - these characteristics make monitoring implementation more challenging as the developers need to deal with interoperability.

It is not part of the company's culture to use UML diagrams or formal artifacts for project specification. Instead, the development teams use informal documents and emails written in natural language to specify the software requirements and communicate ideas. In addition, by the time the case study took place, the project was already specified and the development team was about to start the design and implementation activities. However, in order to provide a brief summary of the project, we have created a use-case diagram with the main use-cases involved, 
as shown in Figure 6.1.

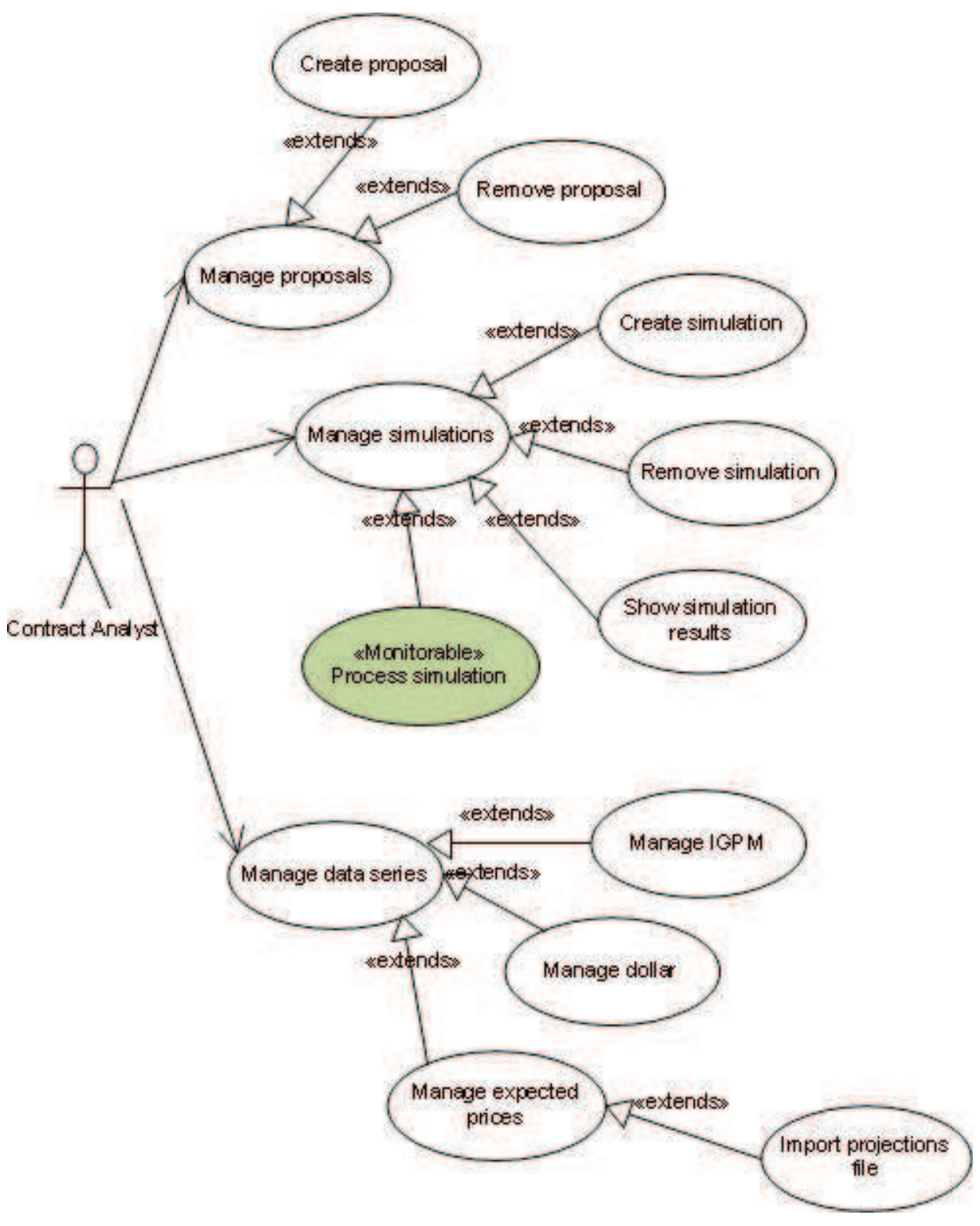

Figure 6.1 Main use-cases of the project under study.

The project is structured in three distinct areas: proposals, data series, and simulations. The first one is concerned with controlling the current proposals sent and received by the traders (possible long-term contracts for buying or selling energy). The second one maintains various market indicators and projections that will serve as input for the mathematical models. The last one is the most relevant for this dissertation and is concerned with processing simulations considering subsets of the available proposals, based on mathematical models that consider a set of business-specific rules, in order to evaluate the risks involved and provide indicators to support the decision to whether or not accept the trading offers.

The only use-case marked with the monitorable stereotype is the Process simulation usecase. It is also the most complex regarding implementation and requires most of the project implementation effort. The use-case consists of processing data and running mathematical models 
to generate information to support the decision of contract analysts. Depending on the simulation configurations, number of proposals and scenarios involved, the simulation process can take from minutes to hours of processing. We do not provide the actual detailed workflow of this use-case due to the following reasons: (1) the use-case is too extensive and full of businessspecific jargons, making it difficult to understand without appropriate training on the brazilian energy market concepts; (2) the mathematical model is an essential part of the client business strategy and it is confidential, so they could not provide the detailed business logic. However, the team leader agreed to provide a simplified version of the use-case activity diagram, aggregating activity details, which does not expose the strategy, but still provides an overview of the use-case activity diagram, which is illustrated in Figure 6.2.

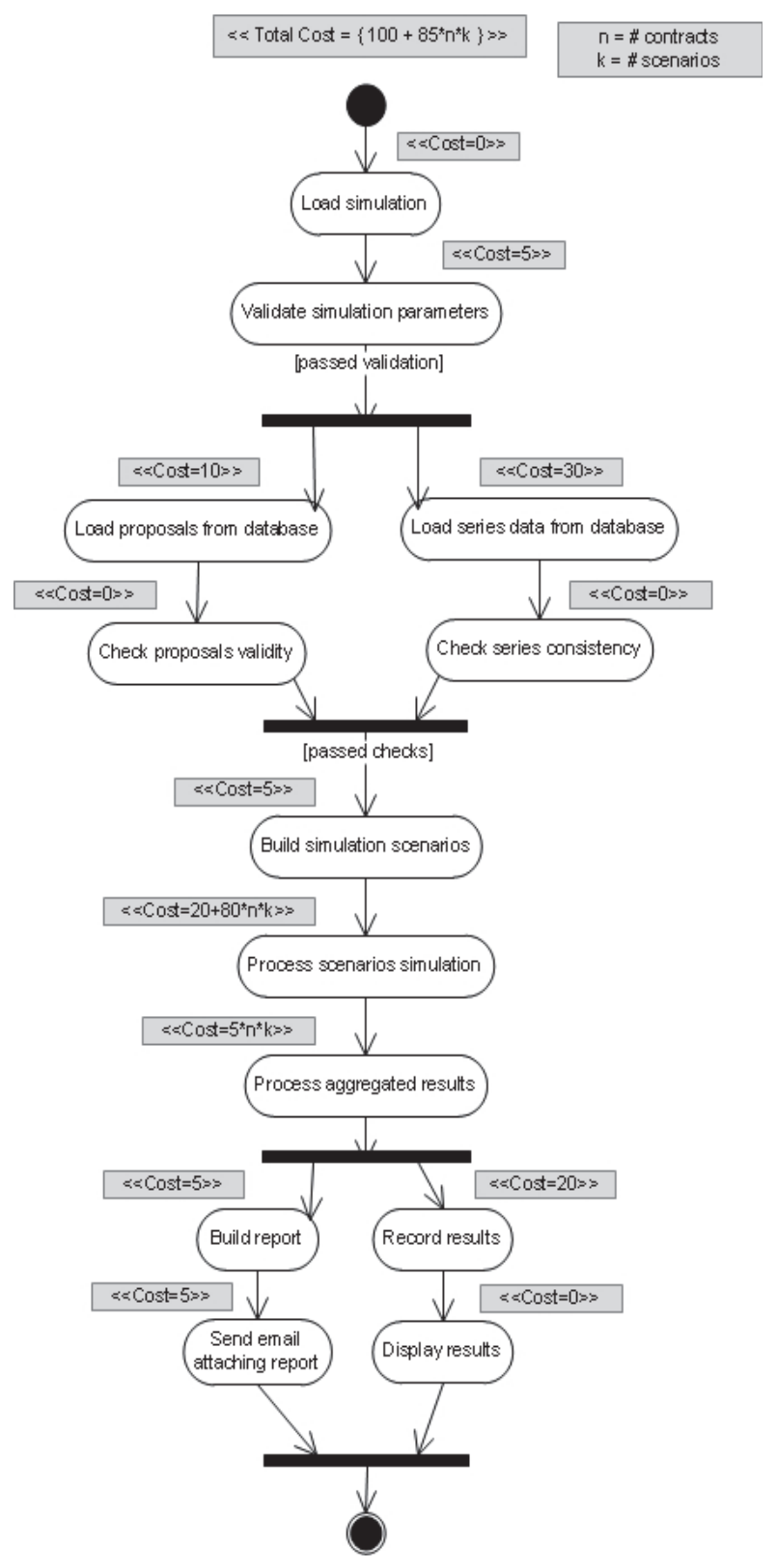

Figure 6.2 Simplified activity diagram of the monitorable use-case. 
The activity diagram provides an overview of the simulation process. However, it is a very high-level simplification that hides the actual implementation details and complex algorithms that take place in the execution process. Notice that the total cost is not an absolute static number, as it depends upon the variables $n$ and $k$ which are respectively the number of proposals and scenarios involved in the simulation.

\subsubsection{Objectives}

The major objective of the study is to analyze the efficiency of the solution in supporting the implementation of monitorable services, with focus on productivity, code tangling and monitoring consistency and quality. Thus, we can state three specific objectives for this case study: (1) to examine whether the solution aids the monitoring development by reducing the required effort (productivity); (2) to verify the resulting code quality with respect to tangling and the added complexity due to the monitoring implementation (maintainability); and (3) to check whether the resulting monitoring information is consistent and uniform along the execution (monitoring quality).

Before we start to describe the details of the case study, it is important to understand the reason why we chose a case study and not another evaluation approach. According to Eaterbrook et al, in [FS08], the most relevant research methods to software engineering are: controlled experiments, case studies, survey researches, ethnographies and action research.

We have a clear evaluation goal, from which we can draw testable hypotheses, metrics, dependent and independent variables. At first glance, this suggests that we should follow the path of a repeatable controlled experiment. In fact, it would be desirable. However, monitoring implementation only becomes a real concern when in the context of medium to large systems, with thousands of lines of code and hundreds of developer working hours, involving timeconsuming services for which the users expect to receive on-line progress feedback. Therefore, the nature of monitoring concern is not a very typical scenario and its usefulness only appears at certain circumstances that we can only find in a real-life project. Even if we could invent some fictitious project, non-commercial and intended only to evaluate our monitoring solution, it would be certainly very costly and require many development working hours - the next section makes this clear by demonstrating the required working hours for the implementation of some real-life monitorable services. For this reason, the controlled experiment, as a technique, is theoretically suitable for evaluating our solution, but in practice it is not a feasible option. Nonetheless, if the required resources for a controlled experiment were available, it would be performed by using the same guidelines and tools of the case study presented in this chapter.

A case study, on the other hand, provides us with a real-life scenario in the exact circumstances where monitoring can be applied. The process is feasible because monitoring is a real requirement and the developers would need to implement it anyway, with or without the support of our proposed solution. The variables involved are very simple and an industrial environment can fit our purposes.

Baseline. Currently, the company uses the ad-hoc approach described in Section 2.1 for implementing monitorable services, due to a lack of known alternatives even from the most experienced developers. Despite the developers complaints about the effort required to implement monitoring by using the ad-hoc approach, it was explained that the tight schedules along with budgetary constraints and requirement priorities never justified the in-house development of an alternative approach. 
Before we can draw testable hypotheses, we determined some constant values (thresholds) for characterizing what should be considered an acceptable and sufficient results regarding monitoring code tangling, productivity and monitoring quality. The reason for choosing these exact constant values for the thresholds is related to the analysis and sampling of historic data about the company's previous monitoring-related projects, which are detailed in Section 6.2.1. These values were obtained after a careful analysis of historical information regarding the implementation of other previous monitorable functionalities at the same company in its recent past.

Hypotheses. Conforming to the objectives, we established the following null hypotheses and alternative ones. According to [ea00], the null hypotheses are the ones intended to be rejected, while the alternative hypotheses are those intended to be confirmed.

Null hypotheses. The null hypotheses determine: (a) that the time spent on the implementation of monitoring is significant when compared to the overall service implementation time; (b) that the code tangling, measured by the relative amount of LOCS, is degraded by the monitoring implementation; (c) that the monitoring quality, measured by its progress feedback consistency, is not sufficient.

$$
\begin{aligned}
& H_{0 a}: \frac{\mu_{\text {monitoring_implementation_time }}}{\mu_{\text {total_implementation_time }}}>0.015 \\
& H_{0 b}: \frac{\mu_{\text {monitoring_implementation_LOCs }}}{\mu_{\text {total_implementation_LOCs }}}>0.01 \\
& H_{0 c}:\left|\frac{\mu_{\text {estimated_executiontime }}}{\mu_{\text {actual_execution_time }}}-1\right|>0.07
\end{aligned}
$$

Alternative hypotheses. For alternative hypotheses, the time spent on the implementation of monitoring is insignificant when compared to the overall service implementation time; (b) the resulting code tangling is acceptable; (c) the resulting monitoring quality consistency suffices. Thus, we have:

$$
\begin{aligned}
& H_{1 a}: \frac{\mu_{\text {monitoring_implementation_time }}}{\mu_{\text {total_implementation_time }}}<=0.015 \\
& H_{1 b}: \frac{\mu_{\text {monitoring_implementation_LOCs }}}{\mu_{\text {total_implementation_LOCs }}}<=0.01 \\
& H_{1 c}:\left|\frac{\mu_{\text {estimated_execution_time }}}{\mu_{\text {actual_execution_time }}}-1\right|<=0.07
\end{aligned}
$$

Criteria. It is difficult to establish an objective criteria for evaluating the framework effectiveness regarding productivity, maintainability and monitoring quality, because not all benefits of using the framework are objectively measurable. For example, even if the percentage of monitoring LOCs remained the same as in the ad-hoc approach, still we would discuss whether the standardized code patterns introduced can make the monitoring code easier to understand and maintain, despite the number of lines of code.

\subsection{Planning}

The case study was performed during a development iteration of a new module of an existing system. The module involved the processing of risk analysis simulations of possible contract values for the next 120 months by switching between $8 \mathrm{k}$ possible scenarios, each one with thousands of variables. The simulation execution time is very volatile and depends upon the number of selected contracts and their specific characteristics, with the execution ranging from minutes to hours. The main monitorable service is the one that processes the simulation, but as the application is large, layered, distributed and object-oriented, the service implementation involves invoking services on lower-level layers, manipulating different object components, and the monitoring code is inevitably scattered among the execution chain. 
We briefly introduced the team to the monitoring framework and code pattern with one hour of informal explanation. We decided not to invest much time in training and deeply discussing monitoring details because we also need to test the intuitiveness of the solution. In practice, monitoring is often considered a non-critical requirement. Thus, if the framework learn curve required too specialized knowledge, it would not be prioritized by the manager, specially if the team is involved with tight project schedules. The case study hypotheses are based on the following evaluation metrics:

- $M T_{1}$ : Time spent on implementation. This metric is concerned with the time - in hours - spent by developers to implement the monitorable service. For this metric, we computed the time spent on the implementation and testing of the monitorable feature, from the first implementation check-out to the last test check-in. This metric will be used to compare the time spent on monitoring implementation with the total time spent on the overall service implementation. In order to accomplish this clear separation, the developers were asked to develop the entire service logic without monitoring support. The time spent was tracked without monitoring. After the core business logic implementation, they were asked to add monitoring capabilities. This strategy enabled a clear distinction between the time spent on actually implementing monitoring from every other business logic.

- $M T_{2}$ : Number of lines of code (LOCs). This metric is used to denote the size of the development products under consideration, by counting the number of lines of code required by the implementation. We used it to compare the number of lines of monitoring code that remains tangled within the monitorable services core business code. This metric does not count blanks, comments or code block openings and endings, because they do not contribute to complexity. By comparing the number of tangled LOCs related to the monitoring concern and the total number of LOCs for the entire feature, we measure the representativeness of monitoring when compared to the entire feature implementation. One can inquire whether this metric can actually assess code quality. In fact, alone, this metric does not assess code quality in a general sense (understandability, changeability, testability, reusability, etc.). However, we are not interested in measuring all attributes of code quality in a general sense. Instead, we focus on measuring code tangling between monitoring-related and business-specific code. For that specific purpose, the metric was considered sufficient.

- $M T_{3}$ : Execution time. This metric is concerned with the monitorable service execution time - in seconds. We compared the estimated execution calculated by the monitoring framework to the actual execution time. If the costs and progress updates provided by each method during the execution chain is consistent and the involved variables are deterministic, then the the estimated time should not deviate from the actual execution time. In fact, the accuracy of the estimated time depends much upon the developer ability to assign consistent costs, but still we decided to measure this result to ensure the final monitoring implementation was actually conforming to the expectations.

For data gathering and analysis of these metrics, we used three professional tools: Microsoft Team Foundation Server 2010 [Cor10c] — which is Microsoft's source code repository, activity and version control system, helping on tracking and registering the implementation time spent 
by the team; The "code metrics" tool of Microsoft Visual Studio 2010 Ultimate Edition [Cor10b] - which is Microsoft's generic IDE for development, assisting on counting the lines of code of the system under development; and the EQATEC Profiler [EQA11] — which is a professional tool for profiling and analyzing execution time of the methods and call stack during application execution. The team leader was responsible for collecting and summarizing the data gathered from the developers.

\subsubsection{Determining the acceptable criteria and thresholds}

Before we could start to use our solution for monitoring implementation, we would need to establish an objective criteria for determining what "an acceptable monitoring implementation regarding productivity and code quality with respect to tangling" actually means. All we knew was that the ad-hoc approach — which is in fact no approach at all — was definitely not sufficient. The reasons for the ad-hoc approach insufficiency were made explicit by the developer complains and can be summarized as follows:

- Monitoring code quality. With the ad-hoc approach, no code patterns or standards are used. There is no guidance for monitoring code. Its implementation is not planned at all and different developers provide different solutions for adding monitoring to the code. In addition, monitoring calculations are not modularized, resulting in lines of code for calculating monitoring states and providing progress feedback all along the core business code. Monitoring code messes the main business algorithm flow. This can be measured by the number of monitoring-related LOCs tangled within the business code.

- Monitoring implementation time. Different developers spend time thinking about different solutions for every new monitoring situation they are faced with. This consumes precious time that should be allocated to other business implementations. This can be measured by the amount of time spent on the implementation of the monitoring-related portion of the code.

- Monitoring quality. Monitoring quality and final consistency depends upon individual developers competence on implementing each detail of the monitoring progress. In practice, they often happen to simplify monitoring calculations and sacrifice monitoring accuracy in order to simplify their code. This can be measured by comparing the calculated monitoring estimated execution time with the actual service execution time.

At first glance, one idea for evaluating our monitoring solution could be to develop a real project twice: once using the ad-hoc approach and another using our proposed solution. But there are some reasons for discarding this idea:

- Insufficient conclusion. The first thing to notice is that a single sample should not be sufficient to draw any general conclusion about the effectiveness of one approach towards the other. In addition, even if we considered a single sample as a legitimate representative sample for characterizing the average monitoring situation, after comparing the results of the ad-hoc approach with the results of our proposed solution, our best possible conclusion would be something like "our solution is better than the ad-hoc approach". The problem is that the proposed solution is not an attempt to optimize an existing acceptable 
solution (in this case, any kind of better solution would be an interesting result). It is an attempt to find an acceptable solution, having no existing acceptable as reference for comparison. Since the ad-hoc approach is very far from corresponding to our expectations, specially regarding code quality and required implementation effort, then we consider more interesting to try to determine an absolute criteria for what does "acceptable" mean, in terms of thresholds for our inequations, and irrespective of the ad-hoc approach.

- Limited time and resource availability. We are dealing with a real-life company, with project schedules, employees and costs associated to their working hours. Software development is often costly and thus, unfortunately, we cannot ask the team leader to implement the same features twice. The company goals and priorities do not justify such effort, specially when we are dealing with projects with thousands of lines of code.

Due to the given reasons, a more appropriate strategy is to define "acceptable result" objectively in terms of absolute metric values. In order to accomplish this, we have gathered information about the quality and development time of recent company projects involving implementation of monitorable services to some extent, considering the current and the last year, and involving distinct company development teams. We analyzed 8 distinct projects whose results are summarized in Table 6.1.

Table 6.1 Analysis of previous monitorable services based on the ad-hoc approach.

\begin{tabular}{|c|ccc|ccc|ccc|}
\cline { 2 - 11 } \multicolumn{1}{c|}{} & \multicolumn{3}{c}{ Code Quality (LOCs) } & \multicolumn{3}{c|}{ Implementation Time (hours) } & \multicolumn{3}{c|}{ Monitoring Quality (seconds) } \\
\hline ID & $\begin{array}{c}\text { Total } \\
\text { LOCs }\end{array}$ & $\begin{array}{c}\text { Monitoring } \\
\text { LOCs }\end{array}$ & $\%$ & $\begin{array}{c}\text { Total } \\
\text { Time }\end{array}$ & $\begin{array}{c}\text { Monitoring } \\
\text { Time }\end{array}$ & $\%$ & $\begin{array}{c}\text { Estimated } \\
\text { Time }\end{array}$ & $\begin{array}{c}\text { Actual } \\
\text { Time }\end{array}$ & $\%$ \\
\hline$\# 1$ & 7.436 & 512 & $6,89 \%$ & 405 & 18 & $4,44 \%$ & 112 & 134 & $19,6 \%$ \\
\hline$\# 2$ & 5.298 & 281 & $5,30 \%$ & 260 & 14 & $5,38 \%$ & 36 & 43 & $19,4 \%$ \\
\hline$\# 3$ & 36.847 & 1.234 & $3,35 \%$ & 2.350 & 55 & $2,34 \%$ & 443 & 566 & $27,8 \%$ \\
\hline$\# 4$ & 14.780 & 645 & $4,36 \%$ & 790 & 33 & $4,18 \%$ & 44 & 54 & $22,7 \%$ \\
\hline$\# 5$ & 1.699 & 75 & $4,41 \%$ & 175 & 8 & $4,57 \%$ & 123 & 146 & $18,7 \%$ \\
\hline$\# 6$ & 21.422 & 1.358 & $6,34 \%$ & 1.490 & 52 & $3,49 \%$ & 344 & 398 & $15,7 \%$ \\
\hline$\# 7$ & 17.561 & 643 & $3,66 \%$ & 1.105 & 42 & $3,80 \%$ & 28 & 36 & $28,6 \%$ \\
\hline$\# 8$ & 9.816 & 449 & $4,57 \%$ & 495 & 22 & $4,44 \%$ & 76 & 94 & $23,7 \%$ \\
\hline
\end{tabular}

The results displayed in Table 6.1 consist of one row for each sample project developed at the company and the columns are organized in three groups of information that relate to the metrics specified in the previous section:

- Code quality (with respect to tangling). Presents the total number of LOCs of the monitorable service (including all components and dependencies involved in the service execution) and the number of LOCs corresponding to monitoring-related code. The percentage represents the proportion of monitoring-related code compared to the entire service implementation. It is desirable to minimize this percentage by reducing monitoring code tangling. The best result obtained in this sampling is the one of project \#3, with $3.35 \%$ of monitoring-related code. 
- Implementation time. Presents the total work hours required to implement the entire monitorable service (including the lower-level layers and other dependencies), and the work hours required for implementing the monitoring-related code. The percentage represents the proportion of monitoring-related working hours compared to the entire service implementation. It is desirable to minimize this percentage by reducing the required effort to implement monitoring. The best result obtained in this sampling is also from the project \#3, with $2.34 \%$ of the working hours dedicated to the monitoring implementation.

- Monitoring quality. Presents the estimated service execution time calculated during the service execution, in addition to the actual observed execution time. The percentage represents how much the estimated value deviates from the actual time. A good quality monitoring result of a deterministic service execution should allow an accurate estimate of the remaining time and therefore a low percentage of deviation. To obtain these values for each service, we performed 5 execution tests for each project and used the best deviation case of the attempts. Probably not a coincidence, project \#3 presented the one of the worst monitoring quality, and maybe it was the price to pay for a better code quality and implementation time. The best result regarding monitoring quality was the one of project \#6, with a minimum deviation of $15.7 \%$.

We have assigned, for each one of the eight projects analyzed, a different analyst as the responsible for gathering and summarizing the information. For extracting historical information from the version control and time tracking systems, they used the same tools and methods as in the project under study.

If we have chosen the best results obtained on using the ad-hoc approach as reference values for establishing the meaning of "acceptable result" — instead of using an average, or at least removing possible outliers - , it could be considered sufficiently challenging already. But we believe that the "acceptable" should be something even better than the best cases obtained in the ad-hoc approach. Therefore, we have established acceptance thresholds that are at least twice as better as the best samples. This led us to the thresholds of $1.5 \%$ for monitoring LOCs percentage; $1 \%$ for implementation time; and $7 \%$ for monitoring estimates deviation.

Not surprisingly, the ad-hoc approach actually showed undesirable results (high percentages of code tangling and implementation time). This was as expected, because the ad-hoc approach does not stimulate reuse of monitoring-related code, leading each developer to implement monitoring in their own way, and often mixing monitoring code with business logic code.

\subsubsection{Experimental Setup}

In this section, we summarize the steps we performed for this case study:

- Find a project for the case study. A large project (more than 500k LOCs) is needed for the solution to be evaluated. It is also important to involve a development team of at least 5 developers.

- Prepare the infrastructure and tools. Some tools are needed for gathering the data for the metrics are available and properly working. For productivity, use a tool for tracking implementation time and source control, such as Microsoft Team Foundation Server. For code tangling, use a tool for counting lines of code, such as the "code metrics" tool of 
Visual Studio. For the monitoring quality, use a profiling tool for measuring the actual execution times, such as the EQATEC Profiler.

- Establish the goals. Gain sensibility regarding the metrics of productivity, code tangling and monitoring quality. Use the tools to analyze previous projects and draw the hypothesis.

- Train the development team. Contextualize and train the developers regarding the use of the solution. Show some examples and code patterns for implementing monitorable methods. Explain them to implement non-monitorable methods first, and making it monitorable in the end, in order to properly track implementation time.

- Collect and organize the data. Use the tools to collect the data and organize them in centralized location.

\subsection{Result Analysis}

The case study was performed at SUATI, with a team of 7 developers, and regarding the implementation of monitorable services as part of a new module for the company main product, GENE, a system with more than 600k LOCs, implemented on top of the .NET Framework using the C\# programming language. More detailed information is published in a website [Car12].

The monitorable services were part of a module corresponding to nearly 50k LOCs. The set of methods that corresponded to the monitorable service implementation ended with precisely 14,491 LOCs and thus they represent a significant portion of the module (nearly $27 \%$ of the overall module LOCs correspond, directly or indirectly, to the monitorable services implementation). In fact, those services represent the heart of the module, which is to perform the calculations and risk analysis simulations under thousands of generated scenarios.

As expected from large systems with an object-oriented design, the execution of a higherlevel service is based upon the execution of various smaller services on the lower layers. Different objects and instances communicate, exchange data and request processing in order for a service to properly execute. For this reason, the 14,491 LOCs are spread among 1,087 different methods.

During the execution, one method makes call to others and so on, extending the execution call stack. During the service execution, we observed a maximum call stack level of 24 methods in the execution chain. Since there was no recursion, this number was considered high by the company developers, when comparing to the typical call stack length in other previous projects they participated. In fact, the high number of different methods involved in the execution of a service makes the monitoring implementation more complex, because the monitoring code would also be spread upon those methods. Therefore, the chosen project for our case study represented a more challenging sample than a typical project, thus corroborating to an appropriate context.

Once the preliminary information about the project has been presented, let us analyze the results obtained for each of the three metrics under evaluation:

- Code quality (with respect to tangling). The monitoring implementation using our solution required 124 LOCs. In comparison with the total 14,491 LOCs required for the entire 
monitorable service implementation, it represents $0.86 \%$ of the entire code, which is less than the $1.5 \%$ established as our acceptance threshold. Thus, the alternative hypothesis $\mathrm{H}_{1 a}$ holds.

- Implementation time. The entire service implementation required 860 hours spread upon 26 working days. The monitoring-related implementation consumed 5 hours, representing $0.58 \%$ percent of the total effort and less than the $1 \%$ established as our acceptance threshold. Thus, the alternative hypothesis $\mathrm{H}_{1 b}$ holds.

- Monitoring quality. The worst monitoring estimate observed during the service execution predicted the execution to take 941 seconds while it actually took 923 seconds, resulting in a deviation of $1.9 \%$, which is less than the $7 \%$ established as our acceptance threshold. Thus, the alternative hypothesis $\mathrm{H}_{1 c}$ holds.

The case study succeeded in testing the solution, indicating that the brief and informal explanation on how to use the solution, to the developers, has been sufficient, corroborating the intuitiveness and practicability of the solution.

Figure 6.3 shows a screen snapshot of the system considered in the case study, displaying a percent-done indicator while the server processes the simulation. Figure 6.4 shows a screen of the system displaying a summary of the simulation results, after the server processing has actually finished.

\subsection{Chapter Summary}

The results of the experience using our solution on a real-life project with monitorable service showed acceptable quality and productivity regarding the standards that we have established. The developers themselves also acknowledged these results, confirming their satisfaction about the resulting product. It is probably not an optimal solution for the monitoring problem, as we still could not completely modularize the monitoring code and make it totally independent from the business logic implementation, but it may probably represent a significant step towards a more optimized solution. 


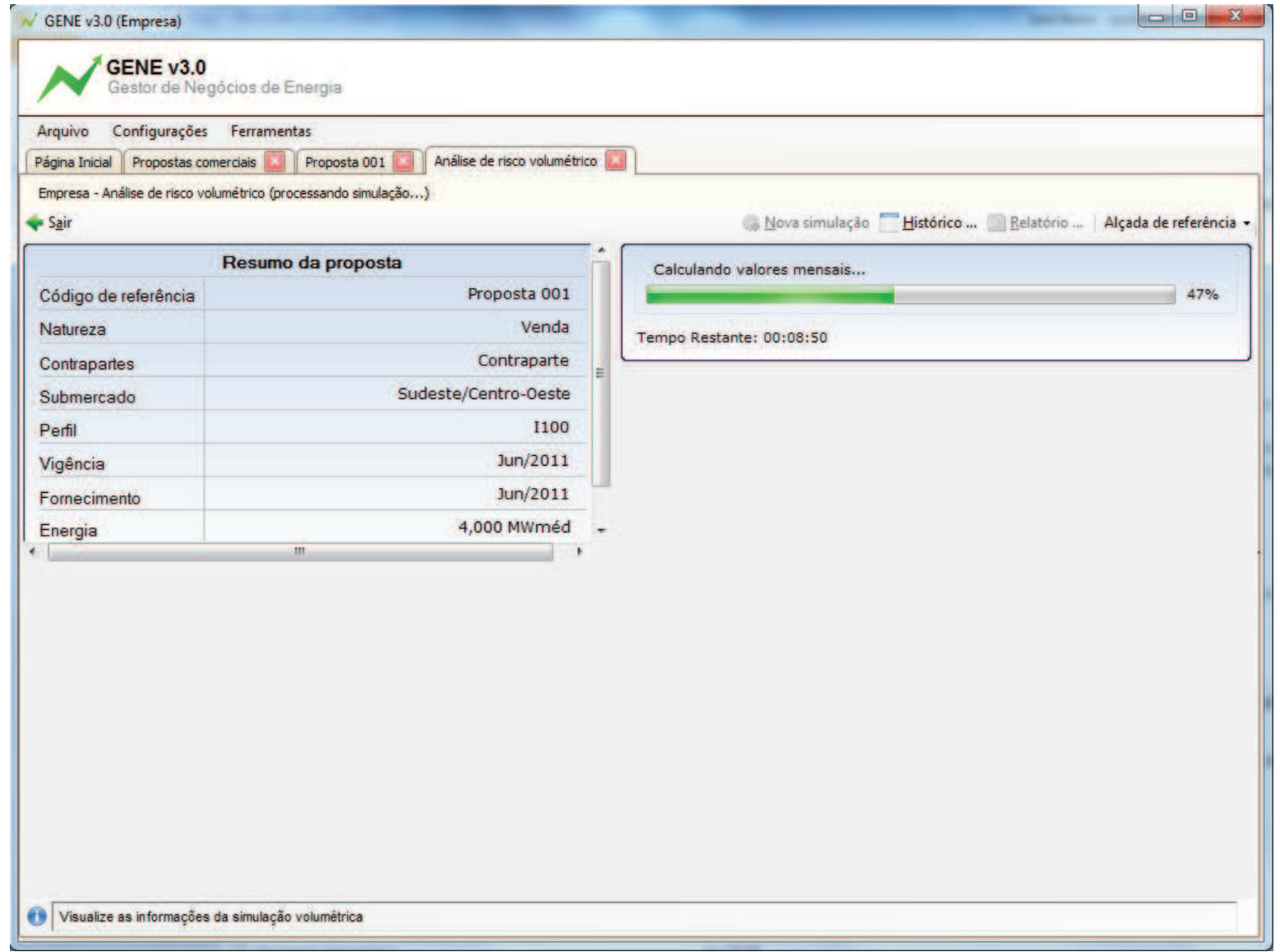

Figure 6.3 Snapshot of the system while the simulation is being processed. 


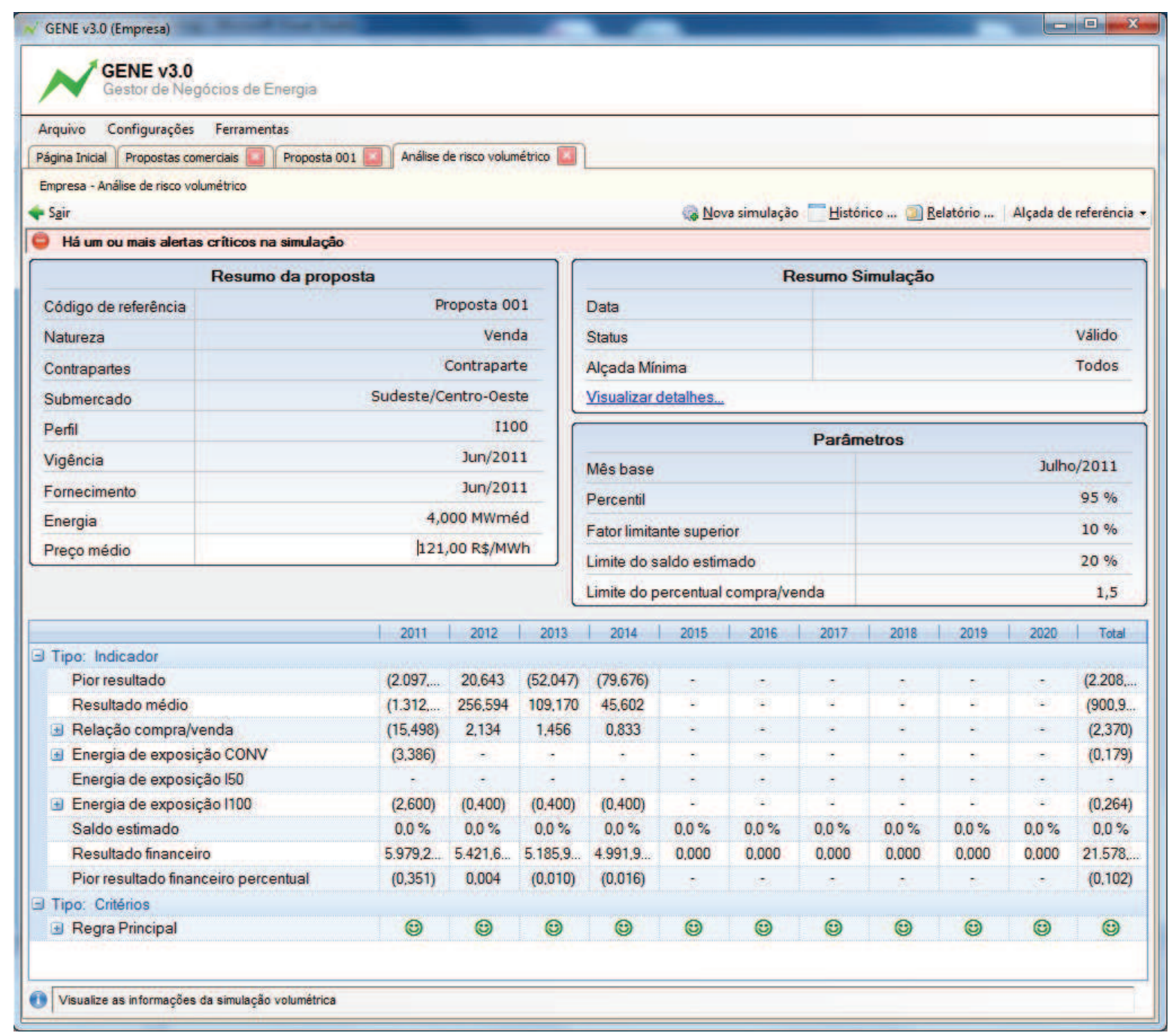

Figure 6.4 Snapshot of the system after the simulation processing has finished. 



\section{CHAPTER 7 \\ Conclusion}

This work proposed and evaluated a solution for supporting the implementation of monitorable services while improving productivity, code quality and monitoring quality, which are the main challenges regarding the monitoring concern. The solution consisted of a general framework for general purpose concepts representation and two specific libraries extending the framework for supporting both the service consumer and provider with concrete implementations. The solution has been evaluated in a software development company, in the context of a real-life project that involved the implementation of monitorable services.

We also presented some possible approaches related to the monitoring concern, detailing the main difficulties involved and analyzing their benefits regarding the implementation of monitorable services. The monitoring problem characterization served as background for the development of this work.

This chapter summarizes the results and draws final considerations. It is organized as follows: Section 7.1 summarizes the achieved goals of the work, while Section 7.2 points out some directions for future work unexplored by this dissertation.

\subsection{Research Contribution}

The main contributions of this work can be summarized as: (1) characterization of the monitoring problem and review of the current approaches regarding its implementation; (2) the definition of requirements, architecture and implementation of a generic extendable framework and two specific concrete extension libraries; (3) an industrial case study that evaluated the solution helpfulness in a real-life large project; (4) Guidelines and patterns towards the impact of monitoring in the overall software development process; and (5) a final product to support monitoring implementation.

- Characterization of the monitoring problem. Through this study, current approaches towards monitoring implementation have been identified and analyzed. This analysis offered the basis to define a set of requirements that should be present in a monitoring solution. Furthermore, the weaknesses and benefits of each approach have been analyzed. Developers involved in monitorable services implementation can understand the main concerns involved in monitoring implementation.

- Framework and libraries specification. After the review and problem characterization, the results and conclusions were the initial step for the definition of requirements for the framework and libraries. Afterwards, its architecture was defined and the framework and libraries implemented. 
- Evaluation. The framework and libraries were used in an industrial large project. The study analyzed the helpfulness in the process, and the findings suggest that the framework usage can aid the developers in the monitoring implementation.

- Impact on software process. A proposition of software process guidelines was presented to be adopted on general software development processes in order to improve the productivity and standardization of the monitoring concern.

- Final product. The final implementation of the framework and libraries offer a full monitoring solution, ready for usage or extension. It is already being used in the company where the case study has been performed, since it has been officially adopted as the monitoring solution and code pattern for their software development.

\subsection{Future Work}

In spite of the commitment to develop the monitoring solution, some possible improvements and related ideas were identified. These possible ideas and improvements are enumerated as follows:

- Code quality optimization. In spite of the progress towards minimizing the amount of code tangling within the monitorable service implementation code, the proposed solution still leads to some monitoring code within the implementation. A perfect monitoring solution should be able to completely eliminate this code tangling. The nature of the problem suggests that an aspect-oriented approach [Kic99] would deal with the tangling problem, as explained in Section 2.3.

- Automatic cost assignment. Costs of operations are manually assigned by the software developers according to the cost of the operation execution compared to the overall service execution. This means the costs are intuitive and thus more subject to human errors. In order to mitigate this problem, we proposed a validation activity after the monitoring implementation that observes the monitoring results and recalibrates the operations costs. Ideally, one could extend the framework to provide static analysis of the code algorithm or alternatively perform profiler-integrated logic at run-time in order to auto-adjust and determine the involved costs. We believe that such an improvement would lead to superior productivity (as the developers would not need to care about cost assignment) and maintainability (as future changes to business code logic would have no impact to the monitoring code because the costs would be auto-adjusted at run-time).

- Programming language constructs. Specific programming language native constructs could be created to deal with the monitoring problem. There are many possibilities to make monitoring more easily implementable, such as (1) static linking of operation costs for methods and code blocks; (2) automatically handling concurrency and monitoring data calculations; (3) native language statements for defining operation execution start and finish. 


\section{Bibliography}

[ADM01] B. R. Helm A. D. Malony. A theory and architecture for automating performance diagnosis. Future Generation Computer Systems, September 2001. 2.6

[API11] Java API. Class jprogressbar. http://download.oracle.com/javase/1,5.0/docs/ api/javax/swing/JProgressBar.html, 2011. 1

[AS00] James R. Trott Alan Shalloway. Design Patterns Explained: A new perspective on Object-Oriented design. Software Patterns Series, dec 2000. 3.2.1

[Bar04] Scott Barber. Beyond performance engineering. developerWorks, jun 2004. 2.6

[Bec07] Kent Beck. Implementation Patterns. Addison-Wesley Professional, 2007. 3.1

[Boe97] Barry Boehm. Managing software productivity and reuse. may 1997. 4.1.3.3

[Car12] David Menezes Cardoso. A framework for monitorable services implementation. https://sites.google.com/site/monitorableservices/, January 2012. 6.3

[Cha10] David Chappel. Introducing windows workflow foundation. http://msdn.microsoft.com/pt-br/library/ee210343.aspx, 2010. 2.5, 2.5, 4.5

[Che06] Nicholas Chen. Convention over configuration. http://softwareengineering.vazexqi.com/files/pattern.html, November 2006. 2.4

[Cor06] Microsoft Corporation. Real world soa. http://www.microsoft.com/biztalk/solutions/soa/whitepaper.mspx, December 2006. 4.3.1

[Cor10a] Microsoft Corporation. C\# Language Specification. Microsoft Corporation, 2010. 4.1.1

[Cor10b] Microsoft Corporation. Microsoft visual studio 2010. http://www.microsoft.com/visualstudio/pt-br/visual-studio-2010-launch, 2010. $5.3 .3,6.2$

[Cor10c] Microsoft Corporation. Microsoft visual studio team foundation server. http://www.microsoft.com/visualstudio/en-us/products/2010-editions/teamfoundation-server/overview, 2010. 6.2 
[Doc10] Eclipse Documentation. Interface iprogressmonitor. http://help.eclipse.org/ helios/index.jsp?topic=/org.eclipse.platform.doc.isv/ reference/api/org/eclipse/core/runtime/IProgressMonitor.html, 2010. 2.2, 2.2

[DS04] Lawrence Wilkes David Sprott. Understanding service-oriented architecture. http://msdn.microsoft.com/en-us/library/aa480021.aspx, January 2004. 4.3.1

[ea00] Wohlin et al. Experimentation in Software Engineering: An Introduction. Kluwer Academic Publishers, 2000. 6.1.2

[ECA08] Beth Plale Eran Chinthaka, Roger Barga and Nelson Araujo. Workflow evolution: Tracing workflows through time. 2008. 2.5

[Ec106a] EclipseMedia. How do i use a subprogressmonitor? http://wiki.eclipse.org/FAQ_How_do_I_use_a_SubProgressMonitor\%3F, aug 2006. 2.2.1

[Ec106b] EclipseMedia. How do i use progress monitors? http://wiki.eclipse.org/ FAQ_How_do_I_use_progress_monitors\%3F, may 2006. 2.2, 2.2

[Ec106c] Eclipse.org. How to correctly and uniformly use progress monitors. http://www.eclipse.org/articles/Article-Progress-Monitors/article.html, jan 2006. 2, 2.2.2

[EQA11] EQATEC. Eqatec profiler. http://www.eqatec.com/Profiler/, 2011. 5.3.3, 6.2

[FCP05] Roger Tourangeau Frederick Conrad, Mick Couper and Andrey Peytchev. Impact of progress feedback on task completion: First impressions matter. CHI 2005, University of Michigan, 2005. 1, 1.3

[FK05] William B. Fakes and Kayo Kang. Software reuse research: Status and future. IEEE Transactions on Software Engineering, 31(7):529-536, july 2005. 1, 1.1

[Fol74] James D. Foley. The art of natural graphic man-machine conversation. Proceedings Fall Joint Computer Conference, pages 462-471, April 1974. 1, 1.3

[FS08] Dag I.K. Sjoberg Forest Shull, Janice Singer. Guide to Advanced Empirical Software Engineering. Springer, 2008. 6.1.2

[GCS07] Marco Scotto Giulio Concas, Ernesto Damiani and Giancarlo Succi. 8th international conference, xp 2007. In Agile Processes in Software Engineering and Extreme Programming, Como, Italy, June 2007. Springer. 5.2.2

[GE95] Vlissides J. Gamma E., Helm R. Design Patterns: Elements of Reusable ObjectOriented Software. Addison-Wesley, 1995. 3.2, 3.2.1, 3.2.2.2, 3.2.4, 3.3

[GF10] Eduardo Guerra and Clovis Fernandes. Architectural patterns for metadata-based frameworks usage. Context, 2010. 2.3, 2.4

[HM00] Kathy Walrath Hans Muller. Threads and swing. http://java.sun.com/products/jfc/tsc/articles/threads/threads1.html, sep 2000. 2.1 
[Jr71] H. Lucas Jr. Performance evaluation and monitoring. Computing Surveys, September 1971. 2.6

[Kic99] Kiczales. Aspect-oriented programming. ACM, July 1999. 1.2, 1.3, 2.1, 2.3, 4.4, 7.2

[Kru92] Charles W. Krueger. Software reuse. ACM Computing Surveys, pages 131-183, 1992. 1.1

[Lib] MSDN Library. Idisposable interface. http://msdn.microsoft.com/enus/library/system.idisposable.aspx. 4.1.1

[Lib08] MSDN Library. Events tutorial. http://msdn.microsoft.com/enus/library/aa645739(VS.71).aspx, mar 2008. 3.2.1

[Lin07] David Linthicum. Service oriented architecture (soa). http://msdn.microsoft.com/en-us/library/bb833022.aspx, 2007. 4.3.1

[Lin09] Henrik Lindberg. Progress monitor patterns. http://henrikeclipse.blogspot.com/2009/05/progress-monitor-patterns.html, may 2009. 2.2.2

[McI68] M. D. McIlroy. Software engineering: Report on a conference sponsored by the nato science committee. Technical report, N. S. A. Division, 1968. 1.1

[Mic06] Brenda M. Michelson. Event-driven architecture overview. Technical report, Patricia Seybold Group, 2006. 4.3.2

[Mil68] Robert B. Miller. Response time in man-computer conversational transactions. Proceedings Fall Joint Computer Conference, pages 267-277, 1968. 1, 1.3

[MPL07] S. Dustdar M. Papazoglou, P. Traverso and F. Leymann. Service-oriented computing: State of the art and research challenges. IEEE, nov 2007. 2.2.1, 4.3.1

[MPL08] S. Dustdar M. Papazoglou, P. Traverso and F. Leymann. Service-oriented computing: A research roadmap. International Journal of Cooperative Information Systems, 2008. 4.3.1

[MW07] Dorina C. Petriu Murray Woodside, Greg Franks. The future of software performance engineering. Future of Software Engineering, 2007. 2.6, 5.3.3

[Mye85] Brad A. Myers. The importance of percent-done progress indicators for computer-human interfaces. CHI'85 Proceedings, 1985. 1

[Net11] Microsoft Developer Network. Introducing microsoft .net framework, 2011. 1, 4.5

[PKG95] M. Jazayeri P. K. Garg. Process-centered software engineering environments. IEEE Computer Society Press, 1995. 5.1 
[PM07] Reidar Conradi Parastoo Mohagheghi. Quality, productivity and economic benefits of software reuse: a review of industrial studies. Empirical Software Engineering, may 2007. 2.1, 2.1, 4.1.3.3

[PN05] S. Graham P. Nibblet. Events and service-oriented architecture: The oasis web services notification specifications. IBM Systems Journal, 2005. 4.3.2, 4.3.2

[Rat99a] Rational. Object-Oriented Analysis and Design Using the UML - Student Manual, Book 1, volume 4.2 - 1. Rational University, 1999. 3.2.1, 5, 5.3.1, 5.3.1

[Rat99b] Rational. Object-Oriented Analysis and Design Using the UML - Student Manual, Book 2, volume 4.2 - 2. Rational University, 1999. 5

[Raw03] Dan Rawsthorne. Comparing/combining rup, xp, and scrum; mixing the process cocktail, March 2003. 5

[RBR93] A. Rajaraman Ramana B. Rao. Method and apparatus for time estimation and progress feedback on distal access operations. Xerox Corporation, April 1993. 1

[Ree79] Trygve M. H. Reenskaug. Thing-model-view-editor an example from a planningsystem. Technical report, XEROX PARC, 1979. 4.3.2

[Sch95] Ken Schwaber. Scrum development process. OOPSLA, 1995. 5

[Sel02] Chris Sells. Safe, simple multithreading in windows forms. http://msdn.microsoft.com/en-us/library/ms951089.aspx, jun 2002. 2.1

[Som07] Ian Sommerville. Software engineering. 8th. Addison-Wesley, 2007. (document), 2.1, 3.1, 5.1, 5.1, 5.2, 5.3, 5.4

[ST05] Latha Srinivasan and Jem Treadwell. An overview of service-oriented architecture, web services and grid computing, nov 2005. HP Software Global Business Unit. 4.3

[Wes99] Mathias Weske. Workflow Management Systems: Formal Foundation, Conceptual Design, Implementation Aspects. $\mathrm{PhD}$ thesis, MathematischNaturwissenschaftlichen Fakultät der Westfälischen Wilhelms-Universität Münster, 1999. 2.5

[Wik10] Wikipedia. Profiling (computer programming). http://en.wikipedia.org/wiki/Profiling_(computer_programming), feb 2010. 2.6

[WVDAB03] B. Kiepuszewski W.M.P. Van Der Aalst, A.H.M. Ter Hofstede and A.P. Barros. Workflow patterns. Distributed and Parallel Databases, 2003. 2.5, 2.5

[XP09] Extreme programming: A gentle introduction. http://www.extremeprogramming.org, 2009. 5 